\title{
Abstracts: XLVI ISCEV International Symposium Morgantown, West Virginia, July 10-15, 2008
}

\section{Oral Session 1: Correlation of cortical structure and function}

\section{Chairs: Michael Bach \& J. Vernon Odom \\ Invited Lecture 1: Studies of visual social cognition}

\author{
Aina Puce \\ West Virginia University Health Sciences Center, \\ Morgantown, WV \\ (No Abstract)
}

\section{OS1-Oral Paper 1}

Multi-harmonic objective response detection in steadystate evoked potentials using permutation techniques

Sven P. Heinrich, Michael Bach

Univ.-Augenklinik Freiburg, Freiburg, Germany

\section{Purpose}

Steady-state evoked potentials are conveniently analyzed in the frequency domain. Usually, only the first harmonic is assessed. In many cases, however, higher harmonics are strong and sometimes even dominant while the first harmonic might be missing completely. It is therefore desirable to use a measure of multi-harmonic significance. Assessing several harmonics separately would require a correction for multiple testing, thereby sacrificing the power of single-harmonic tests but nevertheless effectively ignoring the additional contributions of all harmonics except the one that yielded the smallest $P$ value. In the present study, we evaluate how a conjoint analysis of multiple harmonics based on permutation tests performs in detecting signals of different spectral compositions.

\section{Methods}

We evaluated several different test statistics that can be used as input for the permutation algorithm and compared these to multiple applications of single-harmonic tests. The performance of the tests was assessed with Monte-Carlo simulations using synthetic data that consisted of simulated multi-harmonic responses with different spectral compositions. These responses were embedded into either artificial EEG data or real EEG noise. The false detection rate and the relative sensitivities for a large range of signal-to-noise ratios were assessed for different combinations of harmonics.

Results

With all tests the false detection rate remained within the nominal limit of $5 \%$. Among the statistics tested, the simple sum of harmonic amplitudes showed the best overall sensitivity and outperformed the Bonferroni-corrected multiple tests of single-harmonic significance.

\section{Conclusion}

Permutation-based tests of conjoint multi-frequency significance are a sensitive tool for objective response detection in steady-state evoked potentials. They now await application to pathophysiological questions.

Support

Deutsche Forschungsgemeinschaft (BA 877/18).

\section{OS1-Oral Paper 2}

Attentional shifts have little effect on the waveform of the chromatic onset VEP

Michael A. Crognale, Jennifer Highsmith

University of Nevada Reno, Reno, NV, USA

\section{Purpose}

The chromatic onset VEP has been shown to be a useful diagnostic tool in both congenital and acquired visual defects. However, in some clinical populations it is difficult to control attention, and changes in waveforms with attentional shifts have been reported for both steady-state and multifocal VEPs. Consequently, clinicians and researchers have found it necessary to attempt to monitor the attentional state of the subject, often in a subjective and/or presumptive manner. We have 
noted previously that the chromatic onset VEP appears to be robust to some attentional effects. However, there are numerous stimulus/distractor arrangements in which attention could conceivably modify the VEP response. We report here a series of experiments designed to further test whether or not the chromatic onset VEP can be significantly modified with shifts in attention.

\section{Methods}

The VEP stimuli were $1 \mathrm{cpd}$ sinusoidal gratings presented in an onset/offset mode (100 ms on/400 ms off). Chromatic modulations were chosen to stimulate the opponent pathways and fell along L-M and S-cone axes in color space. Field sizes and stimulus locations varied across experimental conditions. We have explored several different conditions including those in which (1) the stimulus was centrally fixated and attention was directed more peripherally with a distractor task, (2) the stimulus and distractor were presented in different hemifields, (3) the stimulus and distractor were superimposed, and (4) the stimulus and distractor were either similar (task relevant) or dissimilar.

\section{Results}

The waveform amplitude and major component latencies were unaffected by manipulations of attention in the absence of binocular rivalry. These results held true for all the conditions tested.

\section{Conclusion}

The results from this series of experiments suggest that for clinical applications of the chromatic VEP, attentional state does not need to be carefully monitored. It appears that as long as gaze is directed toward the stimulus, the responses will be unaffected by attentional shifts.

Acknowledgments We would like to thank Zenaida Santiago, Chad Duncan, Sean O'Neil, and Eric Roth for help with these experiments.

\section{OS1-Oral Paper 3}

Color and orientation processing in macaque area V2: differential response characteristics defined by single unit electrophysiology and optical imaging

Benjamin M. Ramsden, Clinton G. Cooper

Department of Neurobiology and Anatomy and Sensory Neuroscience Research Center, West Virginia University School of Medicine, Morgantown, WV, USA

\section{Purpose}

Identification of color and luminance contrast within the visual scene is central to both human and non-human primate vision, allowing effective detection and discrimination of behaviorally relevant objects in the surrounding environment. In the second visual cortical area of the primate (Area V2), neurons are known to exhibit preferred firing to various color and oriented stimulus combinations. However, their modulations of temporal response to oriented visual stimuli by color, and its associated mechanisms, are still not well characterized. To investigate these possible modulatory effects, we have been presenting luminance-controlled colored and/or oriented stimuli to anesthetized macaque monkeys during single neuron electrophysiological and intrinsic signal imaging recordings.

\section{Methods}

A chronic chamber preparation allowed comprehensive serial investigations of the same portions of V2 across several months in a cohort of young adult cynomolgus monkeys. The functional organizations of color and orientation response preference were mapped using intrinsic signal optical imaging methods. Subsequent procedures utilized single unit electrophysiology to record spiking response profiles of visually responsive neurons in V2. To quantify the characteristics of neuronal temporal response, we applied several estimation methods including Surprise (Legendy and Salcman 1985) and spike density function (SDF) threshold. These methods provided objective estimates of response latency and allowed quantification of the temporal evolution of response to presentations of oriented, color, and color/oriented stimuli.

\section{Results}

Consistent with previous reports (Munk et al 1995; Shmolesky et al 1998), we observed a broad distribution of response latencies across the population of recorded V2 neurons. Using our multi-stimulus experimental design, we identified colorinduced temporal modulation of oriented response in many neurons. With robust spiking response, Surprise and SDF measures showed comparable latency values; however sensitivity rapidly declined when response was relatively weak. Overall, SDF measures estimated response latency with less variance than Surprise. When oriented stimuli were modulated by color we sometimes observed significant advances in response latency. However, in most cases, the presentation of color led to extended response latency. Pair-wise statistical analysis confirmed a significant latency extension $(P<0.05)$ associated with color modulation across the neuronal group.

\section{Conclusion}

Our experimental results suggest that color/orientation induced response in V2 may arise via a balance of excitatory and inhibitory neurophysiological mechanisms (cf. Anzai et al 2007). Our ongoing studies seek to correlate these observed single unit temporal response characteristics (electrophysiology) with the larger scale functional organization (intrinsic signal imaging).

Support

NIH NCRR RR15574.

\section{Oral Session 2: Insight into retinal function- animal models}

\section{Chairs: Laura Frishman \& Pierre Lachapelle}

\section{OS2-Oral Paper 1}

\section{Transgenic rabbit model of retinal degeneration}

Mineo Kondo ${ }^{1}$, Takao Sakai ${ }^{1}$, Keiichi Komeima ${ }^{1}$, Yukihide Kurimoto $^{1}$, Yuji Nishizawa ${ }^{2}$, Jiro Usukura ${ }^{3}$, Takashi Fujikado $^{4}$, Yasuo Tano ${ }^{4}$, Hiroko Terasaki ${ }^{1}$

${ }^{1}$ Ophthalmology, Nagoya University Graduate School of Medicine, Nagoya, Japan; ${ }^{2}$ Anatomy and Cell Biology, Nagoya University Graduate School of Medicine, Nagoya, Japan; ${ }^{3}$ Materials Physics and Engineering, Nagoya

University Graduate School of Engineering, Nagoya, Japan; 
${ }^{4}$ Ophthalmology, Osaka University Graduate School of Medicine, Suita, Japan

\section{Purpose}

To generate a rabbit that expresses a mutant rhodopsin gene and to characterize the progressive retinal degeneration using RT-PCR, electroretinography (ERG), and histology.

\section{Methods}

The bacterial artificial chromosome (BAC) that contains a rhodopsin gene was identified in a NZW rabbit BAC library. The Pro347Leu mutation of the rhodopsin gene was introduced into the BACs, and these BACs were microinjected into fertilized rabbit eggs. The eggs were then transplanted into 17 recipient rabbits. Transgene-positive animals were identified by Southern blot and PCR analysis. The expression level of the transgene in the retinal tissue was measured by real-time RTPCR. Scotopic and photopic ERGs elicited by different stimulus intensities were recorded. Paraffin retinal sections were stained with hematoxylin and eosin.

\section{Results}

Ten transgene-positive founders were identified, and transgenepositive F1 animals were obtained from 6 of the 10 founders. The expression level of the transgene ranged from 8 to $82 \%$ for the six transgenic lines. ERGs indicated a progressive retinal dysfunction with increasing age in which the rod function was more impaired than the cone function. The ERG abnormality was most severe in the line with the highest transgene expression. Interestingly, some young transgenic rabbits showed hyper-normal OPs. Retinal histology demonstrated a progressive loss of photoreceptors.

\section{Conclusion}

The speed of retinal degeneration was dependant on the expression level of the transgene. Because the rabbits have large eyes and are easy to handle, these animals will be a useful model to study the pathophysiology and treatment of retinal degeneration.

\section{OS2-Oral Paper 2}

\section{Vitamin A-dependent shift of photoreceptor sensitivity in the ERG of Rpe65 mouse mutants}

Naoyuki Tanimoto ${ }^{1}$, Marijana Samardzija ${ }^{2}$, Johannes von Lintig $^{3}$, Vitus Oberhauser ${ }^{3}$, Markus Thiersch ${ }^{2}$, Charlotte E. Reme ${ }^{2}$, T. Michael Redmond ${ }^{4}$, Christian Grimm ${ }^{2}$, Andreas Wenzel ${ }^{1,2}$, Mathias Seeliger ${ }^{1}$

${ }^{1}$ Ocular Neurodegeneration Research Group, Institute for Ophthalmic Research, University of Tuebingen, Tuebingen, Germany; ${ }^{2}$ Laboratory for Retinal Cell Biology, Department of Ophthalmology, University of Zurich, Zurich, Switzerland; ${ }^{3}$ Institute of Biology I, Animal Physiology and Neurobiology, University of Freiburg, Freiburg, Germany; ${ }^{4}$ Laboratory of Retinal Cell and Molecular Biology, National Eye Institute, Bethesda, Maryland, USA

\section{Purpose}

RPE65 is essential for the regeneration of visual pigment by isomerisation of Vitamin A in the visual cycle. Its absence in mice with an Rpe65 null mutation leads to very low levels of 11-cis-retinal and a respective desensitization of photoreceptors (Seeliger et al., Nat Genet 2001). In contrast, mice carrying the R91W loss-of-function mutation in Rpe65 show-on a logarithmic scale-intermediate levels of 11-cis-retinal (Samardzija et al., Hum Mol Genet 2008). The purpose of this study was to compare rod and cone system performance in these two RPE65 mutant lines based on ERG.

\section{Methods}

For this work, R91W knock-in mice, rod opsin (Rho) knockouts (cone function only), R91W/Rho double mutants, RPE65/Rho double knockouts and functionally normal wildtype mice were examined with Ganzfeld electroretinography (ERG; Multiliner Vision, VIASYS, Germany) under both scotopic and photopic conditions.

Results

As the level of RPE65 dysfunction determines the severity of the retinal deficiency, R91W loss-of-function mutants were less affected than knockouts. In agreement with the differences in 11-cis-retinal levels, scotopic ERG recordings revealed that higher flash intensities were needed to induce an electrical response in R91W mice compared to wild-type mice, but lower ones than in RPE65 knockouts. Unexpectedly, photopic ERGs in R91W mice showed a better sensitivity than that in wild-type mice, indicating that the photopic signals-despite the background- were actually mixed responses containing both rod and cone system components. This was verified in R91W/Rho double mutants devoid of any rod contribution. In those, we found that cone sensitivity was reduced relatively to the Rho single knockout to a degree reflecting the reduction in retinoids. Conclusions

In this study, we compared rod and cone system performance in two RPE65 mutant lines. While the ERG sensitivity was reduced by about $3.5-4 \log$ units in the knockout, it was reduced by about $2 \log$ units in R91W. Since the latter reflects roughly the natural sensitivity difference between rods and cones, R91W rods were still active under usual light-adapted conditions and mimicked a cone ERG. Presumably, this is also the case in human patients with loss-of-function mutations in RPE65.

\section{OS2-Oral Paper 3}

\section{Compromised cone system functions in $\mathrm{mdx} 3 \mathrm{cv}$ mice, a model for Duchenne muscular dystrophy}

Bo Lei ${ }^{1,2}$, Keqing Zhang ${ }^{1}$, Yongping Yue ${ }^{3}$, Dongsheng Duan ${ }^{3}$

${ }^{1}$ Veterinary Medicine and Surgery, University of Missouri, Columbia, MO, USA; ${ }^{2}$ Ophthalmology, Mason Eye Institute, University of Missouri-Columbia, Columbia, MO, USA; ${ }^{3}$ Molecular Microbiology and Immunology, University of Missouri-Columbia, Columbia, MO, USA

\section{Purpose}

Duchenne muscular dystrophy (DMD) is an X-linked disorder characterized by progressive muscle degeneration. DMD is caused by mutations in the dystrophin gene. The retinal isoform of dystrophin (Dp260) is localized to the outer plexiform layer. Loss of $\mathrm{Dp} 260$ is believed to result in negative dark-adapted ERG: the rod-driven b-wave is reduced while the a-wave remains unaltered. Little is known about the 
cone system functions in DMD. A recent study indicated that the cone system functions are affected in DMD children. We studied the cone and rod system functions in mdx3cv and mdx $4 \mathrm{cv}$ mice, two distinctive mouse models for DMD.

\section{Methods}

Dark- and light-adapted ERGs were recorded from 1-, 7-, 12-, 16-month-old mdx3cv, mdx4cv, and age-matched C56BL/6J control mice. The stimulus light intensity range covered 6 log units and the background light for light-adapted ERG recording was $30 \mathrm{~cd} / \mathrm{m}^{2}$. OPs were isolated from the composite signals with a digital filter. The frequency range of the filter was set at 60-300 Hz. Retinal morphology was evaluated with light microscope. Profile of the cone photoreceptors was studied with immunohistochemistry by using peanut agglutinin and cone-specific primary antibodies.

\section{Results}

The light-adapted ERG responses in $\mathrm{mdx} 3 \mathrm{cv}$ mice were smaller than those of the wildtype mice. The b-wave amplitudes were approximately $30-50 \%$ of the controls in all age groups. The photopic negative response $(\mathrm{PhNR})$ of $\mathrm{mdx} 3 \mathrm{cv}$ mice was about 1-1.5-fold larger than that of the controls. The dark-adapted ERG b-wave amplitude was significantly reduced while the a-wave was preserved. Consequently, the b-/a-wave ratio was significantly decreased (1 vs. 2). The power of the dark-adapted OP was decreased while the implicit time was prolonged. Morphology and distribution of cone photoreceptors in $\mathrm{mdx} 3 \mathrm{cv}$ mice were comparable to those of the wildtype mice. Mdx4cv mice showed delayed dark-adapted ERG bwave and OPs. However, the cone-driven ERGs and retinal histology appeared normal in $\mathrm{mdx} 4 \mathrm{cv}$ mice.

\section{Conclusions}

Our results suggest that the cone-driven functions are affected in $\mathrm{mdx} 3 \mathrm{cv}$ mice. Interestingly, the $\mathrm{PhNR}$ amplitude was increased in $\mathrm{mdx} 3 \mathrm{cv}$ mice. Because the cone photoreceptors were not affected, the etiology of cone system disorder may likely be post-receptoral. In summary, we have demonstrated that dystrophin mutations may affect both the rod and cone systems.

\section{OS2-Oral Paper 4}

\section{Retinal pathway origins of the pattern ERG (PERG)}

Xunda Luo, Gen Miura, Minhua Wang, Laura J. Frishman

College of Optometry, University of Houston, TX, USA

\section{Purpose}

To investigate and compare the retinal origins of the pattern ERG (PERG) in mice and macaque monkeys using intravitreal pharmacological agents and ganglion cell lesions.

\section{Methods}

PERGs and photopic ganzfeld flash ERGs were recorded from anesthetized C57BL/6 mice 3-4 months of age and anesthetized macaque monkeys 5 to 9 years of age. Recordings were made before and after intravitreal injections of L-APB (2amino-4-phosphonobutyric acid, 1.6-2.0 $\mu \mathrm{M}$ ) to block synapses between photoreceptors and ON-bipolar cells, and TTX (tetrodotoxin, $1-2 \mu \mathrm{M}$ ) to block Na+-dependent spikes. Recordings were also made from mice following optic nerve crush and subsequent degeneration of retinal ganglion cells, and from monkeys with severe monocular experimental glaucoma (Mean Deviation in Humphrey Visual Field 24-2 test worse than $-10 \mathrm{~dB}$ ). For mice, the pattern stimulus on a video monitor consisted of contrast reversing gratings $(1 \mathrm{~Hz})$ of $0.05 \mathrm{cpd}$ presented at $90 \%$ contrast with mean luminance of $100 \mathrm{~cd} / \mathrm{m}^{2}$. For monkeys, the stimulus on the same monitor consisted of a contrast reversing checkerboard of $1 \mathrm{cpd}$ ( 1 or $2 \mathrm{~Hz}$ ) presented at $84 \%$ contrast, with mean luminance of $55 \mathrm{~cd} / \mathrm{m}^{2}$. For both mice and monkeys, effects of pharmacological agents were confirmed in ERG response to ganzfeld flashes presented on a rod saturating background. PERG recordings were made after the effects on flash responses had stabilized ( $\sim 1 \mathrm{~h}$ post injection).

\section{Results}

PERGs in mice had a positive peak (P1) around $61 \mathrm{~ms}$ and maximum negative trough (N2) around $130 \mathrm{~ms}$ after each contrast reversal. L-APB injections removed P1, leaving a negative wave that started earlier than in control eyes, but then followed the time course of N2. Addition of TTX to L-APB injections eliminated almost all of the residual N2. TTX alone left a delayed $\mathrm{P} 1$ and $\mathrm{N} 2$ that were about $25 \%$ and $50 \%$ of control amplitudes, respectively. PERGs in monkeys had a positive peak (P1: counterpart of P50 in humans) around $35 \mathrm{~ms}$ and maximum negative trough (N2: counterpart of N95 in humans) around $105 \mathrm{~ms}$ after each contrast reversal. L-APB injections in monkeys reduced the amplitudes of both P1 and $\mathrm{N} 2$, and addition of TTX eliminated N2. TTX alone also eliminated N2, but a small P1 remained. Retinal ganglion cell lesions in both mice (ONC) and monkeys (experimental glaucoma) eliminated the entire PERG.

\section{Conclusions}

These results confirm previous reports that the PERG primarily reflects ganglion cell activity in both mice and monkeys. However, the PERG in mice is less dependent upon spiking activity than the PERG in monkeys. L-APB results suggest that P1 wave of the mouse PERG is dominated by ganglion cell contributions from the $\mathrm{ON}$ pathway, and $\mathrm{N} 2$ by ganglion cell contributions from the OFF pathway. In monkeys, overlapping contributions from $\mathrm{ON}$ and OFF pathways are more likely. Support

NIH Grant EY06671 (LJF), P30 07551 (UHCO).

\section{OS2-Oral Paper 5}

\section{Photoreceptor contributions to the full field photopic flicker electroretinogram of the rat}

Suresh Viswanathan, Christina Gant

School of Optometry, Indiana University, Bloomington, IN, USA

\section{Purpose}

The purpose of this study was to determine the photoreceptor contribution to the full field photopic flicker ERG of the rat. Methods

Full field photopic flicker ERGs were recorded using DTL electrodes from anesthetized (ketamine $85 \mathrm{mg} / \mathrm{kg} / \mathrm{h}$, i.m. and Xylazine $5 \mathrm{mg} / \mathrm{kg} / \mathrm{h}$, i.m.) pigmented rats (FBNF1/Hsd) using an ESPION system. The visual stimuli consisted of sinusoidally modulated green LED light $(525 \mathrm{~nm}$ peak wavelength 
and $40 \mathrm{~nm} 1 / 2$ band width) with a mean luminance of $500 \mathrm{~cd} / \mathrm{m}^{2}$ and $82 \%$ contrast. The stimulus frequency range was $1-40 \mathrm{~Hz}$. Pupils were fully dilated to about $4 \mathrm{~mm}$ diameter. Responses were obtained in the same eyes before and after blocking postreceptoral response with intravitreal injections of a mixture of $4 \mathrm{mM}$ L-2-amino-4-phosphonobutyric acid (to block depolarizing bipolar cells) and $4 \mathrm{mM}$ cis-piperedine-2, 3-dicarboxylic acid (to block transmission to hyperpolarizing bipolar cells and horizontal cells). Fundamental and second harmonic Fourier components of the flicker ERG responses before and after injection of pharmacological agents were extracted.

\section{Results}

The fundamental as well as the second harmonic Fourier components of the flicker ERG responses from control eyes reduced progressively with increase in stimulus temporal frequency to reach a response minimum around a stimulus frequency of $20 \mathrm{~Hz}$. Thereafter, amplitudes increased slightly with further increase in stimulus temporal frequency, and this increase was relatively larger for the second harmonic compared to the fundamental. The phase of the fundamental and second harmonic components from control eyes showed progressive changes with stimulus frequency up to the frequency that elicited the response minimum and thereafter abruptly shifted to demonstrate a second limb with a steeper slope. Following pharmacological blockade of post-receptoral responses, the fundamental and second harmonic amplitudes were reduced relative to the control amplitudes from the same eyes at all stimulus frequencies greater than $1 \mathrm{~Hz}$. Also, the amplitudes reduced continuously with increase in stimulus temporal frequency with amplitudes reaching noise levels beyond a stimulus frequency of $20 \mathrm{~Hz}$. The phase plots of the fundamental and second harmonic components of the isolated photoreceptor responses were monotonic, consistent with the presence of a single cellular mechanism contributing to these responses.

\section{Conclusions}

Photoreceptors make significant contributions to both the fundamental and the second harmonic Fourier components of the of the full field photopic flicker ERG responses of the rat. These contributions are restricted to stimulus frequencies below $20 \mathrm{~Hz}$. Post-receptoral neurons are largely contributory to the flicker ERG responses at stimulus frequencies greater than $20 \mathrm{~Hz}$. These findings should be useful in the interpretation of the flicker ERGs in different experimental models that employ this species.

\section{Support}

This study was funded by a Faculty Research Support Program grant from Indiana University (SV) and a training grant (T35EY013937) from the Nation Eye Institute (CG).

\section{OS2-Oral Paper 6}

\section{Understanding the origins of the mouse dc-electroretinogram}

Neal S. Peachey ${ }^{1,2,3}$

${ }^{1}$ Research Service, Cleveland VA Medical Center, Cleveland, OH, USA; ${ }^{2}$ Cole Eye Institute, Cleveland Clinic, Cleveland, OH, USA; ${ }^{3}$ Department of Ophthalmology,
Cleveland Clinic Lerner College of Medicine of Case Western Reserve University, Cleveland, $\mathrm{OH}$, USA

\section{Purpose}

To determine the manner in which the major components of the direct current (dc) ERG (dc-ERG) are generated, we recorded responses from mutant mice. This presentation will provide an overview of this work.

\section{Methods}

After overnight dark adaptation, mice were anesthetized with ketamine/xylazine. Responses were recorded from the corneal surface using a pair of $\mathrm{Ag} / \mathrm{AgCl}$ electrodes, making contact with the two eyes. One eye was unstimulated, while the other eye was stimulated with 7-min duration stimuli delivered through a fiber optic bundle; stimulus intensity was controlled using neutral density filters. In some cases, mice were treated with a pharmacological agent prior to the recording session.

\section{Results}

The mouse dc-ERG contains several major components, corresponding to the c-wave, fast oscillation, and light peak, as well as large response at flash offset. All components were retained in nob/Y mice and were abolished in mice lacking rod transducin. All response components were diminished in cftr mutant mice and in rd6/rd6 or rds/+ mice. The c-wave was enhanced in mice expressing one Kir4.1 allele. The light peak was diminished in mice lacking the $\alpha 1 \mathrm{D}(\mathrm{CaV} 1.4)$ or $\beta 4$ subunits of voltage-dependent calcium channels (VDCCs) and by the VDCC blocker nimodipine. The light peak was somewhat larger in mice lacking bestrophin (Best-1).

Conclusions

Mutant mice provide a useful platform for determining the manner in which ERG components are generated.

\section{Poster Session A: Pediatrics-ERG and VEP methods}

Chairs: Anne Fulton \& Thomas Meigen

\section{Poster A1}

The red electroretinogram response, and its relationships with age, gender, body mass index, and axial length, in normal Chinese eyes

Winnie Wai Ying Lau ${ }^{1}$, Albert Chak Ming Wong ${ }^{1}$, Edwin Chan ${ }^{2}$, Clement Wai Nang Chan ${ }^{2}$

${ }^{1}$ Department of Ophthalmology, Queen Mary Hospital, Hong Kong; ${ }^{2}$ Department of Ophthalmology, Tung Wah Eastern Hospital, Hong Kong

\section{Purpose}

To analyze the characteristics of the normal ERG elicited by red light in Chinese eyes, and to determine its relationship with varying age, gender, body mass index (BMI), and axial length. Methods

Flash electroretinography was performed using red light in 238 healthy Chinese patients (476 eyes).

\section{Results}

Three patterns of the red response were observed. The correlation of age was statistically significant for amplitudes 
of scotopic red a waves, scotopic red cone, scotopic red rod, photopic red cone, and with scotopic red rod implicit times and photopic response implicit times of both rods and cones. Correlations between some parameters of the red response with BMI, gender, and axial length were also statistically significant. Conclusions

The values and normal patterns of the ERG red response are described. Its relationships with age, gender, body mass index, and axial length are analyzed. These results provide a reference for future analysis of ERG studies of the red response.

\section{Poster A2}

\section{Early diagnosis of organic amblyopia in children using electroretinography}

\section{S. Mirdehghan, S. M. Shushtarian, M. Mirdehghan}

Tehran Medical Branch, Islamic Azad University, Tehran, Iran

\section{Purpose}

Early diagnosis of amblyopia in children is important as far as proper treatment of amblyopia is concerned. It is the retina and its pathological conditions that may lead to organic amblyopia despite its normal appearance in conventional ophthalmoscopy; therefore, electroretinography was tested in a population of children with amblyopia to check for probable malfunctioning of retina.

\section{Methods}

Fifty children with diagnosis of amblyopia and unsuccessful patching treatment underwent electroretinography examination. Conventional electrodes attachment was used to record ERG. The ERG records of patients were compared with 50 ERG records of children with no sign of amblyopia.

\section{Result}

$30 \%$ of the patients had broad ERG b waves which are an indication of early retinal changes.

\section{Conclusion}

From the present study one can conclude that ERG is a suitable technique to look for organic amblyopia which may arise from the retina in these patients.

\section{Poster A3}

\section{Recording of electroretinogram and visual evoked potential under anaesthesia}

Mohammad Mehdi Shushtarian ${ }^{1}$, Seyed Mohammad Masoud Shushtarian ${ }^{2}$, Soheil Feizi ${ }^{3}$, Tahereh Parsa ${ }^{3}$, Alireza Khodyani ${ }^{2}$

${ }^{1}$ Iran University of Medical Science, Tehran, Iran; ${ }^{2}$ Tehran Medical Branch, Islamic Azad University, Tehran, Iran; ${ }^{3}$ Shahid Beheshti Medical University, Tehran, Iran

\section{Purpose}

Recording of bio potentials under anaesthesia is an important task in certain cases when the ordinary recording is impossible. Recording of bio potentials in infants is impossible because of lack of cooperation; therefore, in the present study; fifty infants underwent ERG and VEP testing under anaesthesia. The two diagnostic techniques were compared with normal patterns to check for the reliability of the recording obtained.

\section{Methods}

Fifty infants referred to Qods polyclinic were selected randomly. ERG and VEP were recorded in total population. Conventional electrode attachment was used for related recordings. The patterns obtained were compared with normal ERG and VEP patterns obtained in normal subjects who underwent ERG and VEP recording without anaesthesia.

\section{Result}

The results show that the reliable ERGs but not VEPs were recordable; i.e. the ' $a$ ' and ' $b$ ' waves were distinct in ERGs, whereas no reliable VEP P100s were recorded in most of the population under anaesthesia. The reason for this discrepancy may be the fact that, the ERG is the integration of few layers of retina, whereas the VEP is the integration of large number of neuronal layers. Other aspects will be discussed.

\section{Conclusion}

From the result of present work, one can conclude that ERG patterns are more reliable than VEP patterns under anaesthesia.

\section{Poster A4}

$30 \mathrm{~Hz}$ and mixed response electroretinogram done during anaesthesia as predictors of visual outcome in children affected by various eye diseases: 10 years survey

Giulio Ruberto ${ }^{1}$, Rosanna Guagliano ${ }^{1}$, Donatella Barillà ${ }^{1}$, Mauro Antonimi ${ }^{2}$, Sara Pezzotta ${ }^{1}$, Sabrina Signorini ${ }^{2}$, Elisa Fazzi ${ }^{2}$, Raffaella Angeli ${ }^{1}$, Carmine Tinelli ${ }^{3}$, Paolo Emilio Bianchi ${ }^{1}$

${ }^{1}$ Eye Clinic IRCCS San Matteo Hospital, Pavia, Italy; ${ }^{2}$ Department of Child Neurology and Psychiatry-Regional Centre of Child Neuroophthalmology, IRCCS C. Mondino Institute of Neurology, Pavia; ${ }^{3}$ Biometrics Service, IRCCS San Matteo Hospital, Pavia

\section{Purpose}

This study aimed to define the relationship between $30 \mathrm{~Hz}$ and mixed maximal response ERG recorded under anaesthesia and the visual outcome in subjects affected by various retinal and/ or optic disk affections.

\section{Methods}

We present a review of 87 among 260 children examined under anaesthesia, because not otherwise surveyable, in the last decade. All subjects, mean age 3.12 [2, 3], underwent biomicroscopy, fundus examination, ERG and flash visual evoked potentials. The electroretinography was performed without adaptation in a mid-luminance only with $30 \mathrm{~Hz}$ and mixed maximal response to shorten the time of the anaesthesia. Erg jet corneal electrodes were placed on the eyes after dilatation with tropicamide drops. A supple flash head was placed $30 \mathrm{~cm}$. above the child's head. General anaesthesia was induced in the first minute with a mixture of $\mathrm{O}_{2} /$ air $50 \%$ enriched with nitrogen protoxide $\left(\mathrm{N}_{2} \mathrm{O}\right)$, and afterward a mixture of $\mathrm{O}_{2} /$ air $50 \%$ enriched with $3 \%$ sevofluorane. The collected results were matched with the fundus findings and the visual acuities in the 87 children that was possible resurvey in the Ophthalmic Clinic or in the Department of Child 
Neurology and Psychiatry. Multiple statistical analysis comprehending Student's $t$-test for independent samples was used for comparisons between groups. A $P$-value of less than 0.05 was assumed to indicate statistical significance.

\section{Results}

Diagnosis linking fundus findings and electroretinography were: 7 Leber dystrophy, 12 Retinopathy of prematurity, 20 retinal dystrophy, 6 nystagmus, 5 cerebral visual impairment, 2 Joubert syndrome, 1 Retinoblastoma, 5 optic nerve coloboma, 1 Morning glory syndrome, 12 pale papilla, 2 congenital cataract associated with retinal anomalies, 14 not defined. Mean visual acuity was 1.94 in the right eye, and 2.04 in the left. Highly significant correlations and " $P$ " values $<0.05$ were found between $30 \mathrm{~Hz}$ amplitude, after fast Fourier analysis, a and b amplitudes, b latency and visual acuities. The most-strict correlation was found with retinal dystrophy. In the same category, a significant $r$ coefficient was found.

\section{Conclusions}

Our work indicates a strict correlation between fundus findings, electroretinography and visual outcome. When we are unable to perform a correct diagnosis by visit and instrumental methods in awake conditions, a brief anaesthesia allows one to obtain results which would otherwise be arduous. We had no adverse effects in our sessions.

\section{Poster A5}

\section{Comparison of electroretinographical patterns in normal children and adult population}

\author{
H. Hassani ${ }^{1}$, S. M. Shushtarian ${ }^{2}$ \\ ${ }^{1}$ Islamic Azad University, Tehran, Iran; ${ }^{2}$ Tehran Medical \\ Branch, Tehran, Iran
}

\section{Purpose}

Recording of ERG is important for accurate diagnosis in many conditions. ERG is difficult to record in children as they do not cooperate fully. ERG was recorded in fifty relatively cooperative children and compared with the patterns obtained in adult population to evaluate any differences between the two groups. Methods

Fifty normal children and fifty normal adults underwent ERG testing. Conventional electrodes were used in all subjects. ERG patterns obtained in the two groups were compared.

\section{Results}

The results obtained show a slightly broader b-wave in children compared to the adult group.

\section{Conclusions}

It is reported that broad b-wave represents early retinal changes whereas, in children, the broad b-wave may be due to lack of cooperation.

\section{Poster A6}

\section{Chromatic VEP in the first year of life-a longitudinal study}

Manca Tekavcic Pompe, Branka Stirn Kranjc, Jelka Brecelj

Eye Clinic, University Medical Centre, Ljubljana, Slovenia

\section{Purpose}

To study development of VEP responses to red-green and blueyellow chromatic stimulus longitudinally during the first year of life.

\section{Methods}

A baby girl was binocularly tested every month during the first year of life. VEP were recorded to isoluminant red-green and blue-yellow stimulus. The stimulus was circle composed of horizontal sinusoidal gratings with $90 \%$ chromatic contrast. Two spatial frequency values $(0.5 \mathrm{cpd}$ and $2 \mathrm{cpd})$ and two stimulus sizes ( $7 \mathrm{deg}$ and $21 \mathrm{deg}$ ) were used. The stimulus was presented in an onset-offset mode (on-300 ms, off-700 ms). VEP were recorded from $\mathrm{Oz}$ (mid occipital) position and the reference was at Fz. Waveform and its changes throughout the first year were studied.

\section{Results}

At the age of 31 days there was no response to red-green and blue-yellow stimulus, regardless of stimulus size and spatial frequency. At the age of 53 days VEP response was recordable to large $\left(21^{\circ}\right)$ stimulus with spatial frequency $0.5 \mathrm{cpd}$. The response consisted of predominantly positive component that showed higher amplitude to red-green in comparison to blueyellow stimulus. No VEP response was detected to smaller $\left(7^{\circ}\right)$ stimulus with spatial frequency $2 \mathrm{cpd}$ regardless of stimulus color. At the age of 126 days VEP response was detected to all red-green and blue-yellow stimuli (both stimulus sizes and spatial frequencies) and consisted of predominantly positive component, which continued to change (in amplitude and latency) until last recording, performed at the age of 394 days. Conclusions

VEP responses to chromatic stimulus can be recorded at younger age to red-green than to blue-yellow stimulus as well as to larger stimulus size and smaller spatial frequency than to smaller stimulus size and higher spatial frequency. These findings seem to be in line with psychophysical studies and visual process maturation.

\section{Poster A7}

\section{Neonatal flash VEPS: effect of exposure to methadone} in utero

Ruth Hamilton ${ }^{1}$, Laura McGlone ${ }^{2}$, Helen Mactier ${ }^{2}$, Michael Bradnam ${ }^{1}$, Richard Boulton ${ }^{3}$, William Borland ${ }^{4}$, Mary Hepburn ${ }^{2}$, Daphne McCulloch ${ }^{5}$

${ }^{1}$ Department of Clinical Physics, Royal Hospital for Sick Children and University of Glasgow, Glasgow; ${ }^{2}$ Neonatal Unit, Princess Royal Maternity, Glasgow; ${ }^{3}$ Department of Clinical Physics, Princess Royal Maternity and University of Glasgow, Glasgow; ${ }^{4}$ Department of Biochemistry, Gartnavel General Hospital, Glasgow; ${ }^{5}$ Vision Sciences, Glasgow Caledonian University

\section{Purpose}

To compare flash VEPs recorded in the first few days after birth between infants exposed in utero to methadone and control infants.

\section{Methods}

21 term-born ( $\geq 37$ weeks gestation) infants born to mothers prescribed methadone during pregnancy and 20 control infants 
were recruited. In utero drug exposure was determined from maternal history and infant urine analysis. 12 of the 21 (57\%) methadone-exposed infants had also been exposed to one or more of the following drugs: opiates (heroin) $(N=12)$; benzodiazepines $(N=8)$; cocaine $(N=1)$; cannabis $(N=1)$. Flash VEPs were recorded in the first four days of life using a hand-held integrating sphere $\left(\right.$ Colorburst $^{\circledR}$, Diagnosis, UK) delivering $50 \mathrm{~cd} \mathrm{~s} \mathrm{~m}^{-2}$ flashes. VEPs were classified as present or absent based on reproducibility. Disregarding polarity, a total amplitude measure was obtained by summing peak and trough amplitudes. If present, peaks and troughs were labelled in order of increasing implicit time as P1, P2, N3, P3 and individual amplitudes from baseline and implicit times noted.

\section{Results}

20/20 (100\%) of control infants had a reproducible flash VEP compared with $17 / 21(81 \%)$ of the methadone-exposed infants. Total amplitude medians were $30 \mu \mathrm{V}$ for the control group and $17 \mu \mathrm{V}$ for the methadone-exposed group: comparing the groups supports the hypothesis that population medians differ (Mann-Whitney U-test, 95\% CI of difference 5-25 $\mu \mathrm{V}$, $\mathrm{W}=531.5, P=0.002)$. N3 was present significantly more often in the control group than in the methadone-exposed group (20/20 versus 16/21, 95\% CI of difference 3-45\%): prevalence of peaks P1, P2 and P3 did not differ significantly between groups. When present, the P2 peak for methadoneexposed infants had longer mean implicit times than for controls: (240 ms versus $189 \mathrm{~ms}, P=0.004)$. Amplitudes of $\mathrm{P} 1, \mathrm{P} 2$ and N3 were smaller for methadone-exposed infants than controls: $\mathrm{P} 1,3 \mu \mathrm{V}$ versus $5 \mu \mathrm{V}(P=0.02)$; $\mathrm{P} 2,18 \mu \mathrm{V}$ versus $14 \mu \mathrm{V} \quad(P=0.009) ; \quad \mathrm{N} 3, \quad 16 \mu \mathrm{V}$ versus $10 \mu \mathrm{V}$ $(P=0.045)$. P3 amplitude did not differ between groups.

\section{Conclusions}

These data show a marked difference in the neonatal flash VEP of infants exposed to methadone in utero compared with their non-exposed peers. In general, the VEPs are more likely to be absent and, if present, have smaller overall amplitude, show fewer N3 troughs and longer P2 implicit times. This objective analysis complements qualitative analysis of the same waveforms (McGlone L et al (2008) Visual evoked potentials in infants exposed to methadone in utero. Arch Disease Childhood).

\section{Poster A8}

\section{Electrophysiological impairment is associated with biochemical and histological modifications in the retina of chronic alcoholic rats}

M. Sancho-Tello ${ }^{1}$, M. Muriach ${ }^{2}$, J. Barcia ${ }^{2}$, F. Bosch-Morell ${ }^{2}$, J. M. Genovés ${ }^{2}$, S. Johnsen-Soriano ${ }^{1}$, B. Romero ${ }^{2}$, I. Almansa ${ }^{2}$, M. Díaz-Llopis ${ }^{3}$, S. García-Delpech ${ }^{3}$, J. Romá $^{4}$, C. Vilela ${ }^{1,5}$, F. J. Romero ${ }^{1,2}$

${ }^{1}$ Fundación Oftalmológica del Mediterráneo, Valencia, Spain; ${ }^{2}$ Instituto sobre Drogas y Conductas Adictivas, Universidad CEU Cardenal Herrera, Valencia, Spain; ${ }^{3}$ Dept. Cirurgia and ${ }^{4}$ Dept. Fisiologia, Universitat de València, Valencia, Spain; ${ }^{5}$ Dept. Neurofisiología Clínica, Hospital Universitario La Fe, Valencia, Spain

\section{Purpose}

Ethanol consumption originates a wide spectrum of disorders, including alteration of visual function. Oxidative stress is included among the mechanisms by which alcohol predisposes nervous tissue to injury. Retina, which is the neurosensorial eye tissue, is particularly sensitive to oxidative stress.

\section{Methods}

In this study we analyze the effect of long-term alcohol consumption on oxidative stress parameters of the rat retina, and its correlation to retinal function, as well as to the expression of the antiapoptotic protein $\mathrm{Bcl}-2$. We also study the protective effect of ebselen, a synthetic selenoorganic antioxidant.

\section{Results}

Herein we show that ethanol has a toxic effect on rat retina associated with oxidative stress. Decreases in retina glutathione concentration and increases in malondialdehyde content in whole eye homogenate significantly correlate with ERG bwave decrease and Bcl-2 overexpression. We also show how ebselen is able to prevent all the alterations observed.

\section{Conclusion}

Chronic ethanol consumption induces oxidative stress in rat retina associated with an impairment of ERG and Bcl-2 overexpression, suggesting a role for glial cells. All these alterations in the rat allow the proposal of an 'alcoholic retinopathy' in this species.

\section{Support}

Dirección General de Drogodependencias, Generalitat Valenciana, and grant SAF2007-66801.

\section{Poster A9}

\section{Photopic on- and off-responses in normal Korean subjects}

Soo Young Lee ${ }^{1}$, Su Eun Park ${ }^{2}$, Young-Hoon $\mathrm{Ohn}^{2}$

${ }^{1}$ Department of Ophthalmology, School of Medicine, Ewha

Womans University, Seoul, Korea: ${ }^{2}$ Department of Ophthalmology, Soonchunhyang University Hospital, Bucheon, Korea

\section{Purpose}

To investigate the characteristics of photopic on- and offresponse using long duration stimulation and to standardize the duration and intensity of stimulation, and background luminance for clinical ERG recording.

\section{Methods}

Photopic on- and off-responses were recorded on 20 eyes of 20 normal subjects using contact lens electrode with built-in white LED stimulator (MAYO Corporation, Aichi, Japan). Pupils were fully dilated with $0.5 \%$ tropicamide and phenylephrine hydrochloride and tests were performed after $10 \mathrm{~min}$ of light adaptation. The influence of stimulation duration on amplitude was evaluated with flash duration varying from 5 to $200 \mathrm{~ms}$. The influence of stimulus intensity was studied with $0.4,0.7$, $1.1,1.4,1.7,1.9,2.0$, and $2.1 \mathrm{log} \mathrm{cd} / \mathrm{m}^{2}$ and the influence of background luminance with $20,30,40$, and $50 \mathrm{~cd} / \mathrm{m}^{2}$.

\section{Results}

The b- and d-waves were separated from the stimulation of $50 \mathrm{~ms}$ in 16 eyes and separated at the stimulation of $100 \mathrm{~ms}$ in all 
subject eyes. The maximum amplitude of b-wave was noticed at the stimulation of $100 \mathrm{~ms}$ (mean $69.3 \mu \mathrm{V}$ ), and d-wave was noticed at the stimulation of $200 \mathrm{~ms}$ (mean $50.09 \mu \mathrm{V}$ ). The amplitudes of $b$ - and d-waves were increased as stimulus intensity was increased and reduced as background luminance was increased.

\section{Conclusion}

These results suggest that optimal parameters are stimulus duration of $100-150 \mathrm{~ms}$, intensity of $1.7-2.1 \mathrm{log} \mathrm{cd} / \mathrm{m}^{2}$, under background luminance of $30-40 \mathrm{~cd} / \mathrm{m}^{2}$ for clinical photopic ERG recording.

\section{Poster A10}

\section{Changes of retinal adaptation in myopic eye}

Wing-cheung Ho, Yiu-fai Ng, Patrick Ho-wai Chu, Ying-ying Fong, Kwun-sum Yip, Henry Ho-lung Chan

Laboratory of Experimental Optometry (Neuroscience), School of Optometry, The Hong Kong Polytechnic University, Hung Hom, Kowloon, Hong Kong SAR, China

\section{Purpose}

To investigate the functional changes in myopic eyes by using mfERG with global flash paradigm at moderate and high luminance difference levels.

\section{Methods}

Ten low myopes (spherical equivalent refractive error ranged from plano to $-3.00 \mathrm{D}$ ) and 10 high myopes (spherical equivalent refractive error greater than $-6.00 \mathrm{D}$ ), who were age-matched $(20.9 \pm 1.4$ compared to $21.6 \pm 1.3$ years $)$, without any signs of pathological myopia underwent a multifocal electroretinography examination with global flash paradigm consisting of four sequential video frames: a pseudorandom $\mathrm{m}$-sequence stimulation, a dark frame, a global flash and a dark frame again. For the pseudo-random m-sequence stimulation, the luminance difference levels were set at 1.08 $(49 \%)$ and $2.12(96 \%) \mathrm{cd} \mathrm{s} / \mathrm{m}^{2}$. The amplitude and implicit time of direct component (DC) and induced component (IC) from central (retinal area of central $19^{\circ}$ ) and peripheral region (retinal area from $19^{\circ}$ to $48^{\circ}$ ) were measured for analysis. Two-way ANOVA was performed to investigate any differences in amplitude or implicit time between the two groups of subjects.

\section{Results}

There was a significant linear relationship between refractive error and axial length, indicating most of our subjects were axial myopia (Pearson's correlation: $r=-0.909, P<0.0001$ ). The IC correlated with the axial length significantly at $96 \%$ level in the central region (Pearson's correlation: $r=-0.532$, $P=0.019$ ) but not in the peripheral region (Pearson's correlation: $r=-0.398, P=0.091)$. There was no significant correlation between IC and axial length at $49 \%$ level either in the central region (Pearson's correlation: $r=-0.127$, $P=0.604$ ) or in the peripheral region (Pearson's correlation: $r=-0.273, P=0.258$ ). Compared to low myopes, the amplitude of DC in high myopes did not have significant changes while the IC response was significantly attenuated at both $49 \%$ and $96 \%$ levels in the central region; the implicit time of DC and IC did not have a significant difference between low and high myopes. In addition, there were no significant differences in either the amplitude or the implicit time of DC and IC in the peripheral region.

\section{Conclusions}

While it is suggested that the adaptive mechanism mainly takes place at the retinal post-receptoral level, the reduced IC, which reflects the adaptation activity at inner retinal layers, was impaired in high myopes and likely limited to the central retina presumably due to altered synaptic connection within retinal layers as a result of axial elongation.

\section{Support}

Competitive Earmark Research Grants (PolyU 5384/04M and PolyU 5415/06M) from The Research Grants Committee of The Hong Kong SAR, the Niche Areas-Glaucoma Research (1-BB76) and the Associated Fund (Research Postgraduate) from The Hong Kong Polytechnic University.

\section{Poster A11}

Would increasing flash intensity be sufficient to yield similar ERG amplitudes in the nondilated eye compared to the dilated eye?

Marc Hébert, Marie-Pier Lavoie, Marie-Claude Charron, Alexandre Sasseville, Anne-Marie Gagné

Centre de Recherche Université Laval Robert-Giffard, Université Laval, Québec, Canada

\section{Purpose}

We investigated the possibility of performing ERG in nondilated eyes using brighter flashes.

\section{Methods}

Photopic $\left(N=26\right.$, background $\left.25.5 \mathrm{~cd} / \mathrm{m}^{-2}\right)$ and scotopic ERGs ( $N=23$, green flashes) luminance response functions were obtained simultaneously in a pharmacologically dilated (DE) and nonpharmacologically dilated eye (NDE). Recordings were performed with DTL electrodes and acquired with the Espion-Color dome (E2, Diagnosys LLC).

\section{Results}

Compared to the DE, the NDE photopic $V_{\max }$ b-wave amplitude was reduced by $14 \%(P<0.0001)$, implicit time prolonged $(P<0.0001)$ and retinal sensitivity $(\log \mathrm{K})$ decreased by $0.38 \log$ units $(P<0.0001)$. Increasing the background to $0.50 \log$ units $\left(80 \mathrm{~cd} / \mathrm{m}^{2}\right)$ appeared to correct for implicit time but not for amplitude. Rod $V_{\max }$ was decreased by $7 \%$ in NDE $(P<0.05)$ and retinal sensitivity decreased by $0.40 \log$ units $(P<0.0001)$ but implicit time was not affected.

\section{Conclusions}

By simply increasing flash intensities, we were not able to achieve similar maximal amplitudes between the nonpharmacologically dilated eye and the pharmacologically dilated eye. As would be expected, differences were less important in the scotopic ERG since the eye is naturally dilated in the dark. This raises the question as to whether it would be possible to adjust flash intensity according to pupil size in order to optimize the stimulation of the entire retina. 


\section{Poster A12}

\section{Effect of docosahexaenoic acid supplementation on retinal function in ELOVL4 mice}

S. C. Mema, S. Kuny, F. Gaillard, K. Zhang, I. M. MacDonald, M. T. Clandinin, Y. Sauvé

University of Alberta, Calgary, Alberta, Canada

\section{Introduction}

The ELOVL4 gene is thought to be linked with the production of DHA, which humans can synthesize but only in small amounts. Functional improvement in vision has been reported in patients with a mutation in ELOVL4 who were given DHA in their diet. For this study we have examined how DHA supplementation might delay functional decline both in ELOVL4 and wt mice from 1 to 3 months of age.

\section{Purpose}

To assess whether a high DHA diet will prevent loss of retina function in ELOVL4 transgenic mice in comparison to littermate mice fed a non-DHA diet.

\section{Methods}

ELOVL4 mice $(n=11)$ and wild type (wt) littermates $(n=13)$ at one month of age (line 2 from Dr. K Zhang, Moran Eye Center, Utah) were each divided into two groups, one fed a $0.44 \%$ DHA diet (E4+, WT+), the other fed the same diet without DHA (E4-, WT-). Under xylazineketamine anesthesia, ERGs were recorded (Espion E2, Diagnosys LLC). After two months of dietary manipulation standard ISCEV bright flash and photopic tests were recorded, as well as the cone specific test scotopic doubleflash ERG in which an initial flash bleached the rods, leading to isolated cone responses to a second flash delivered $800 \mathrm{~ms}$. later. In order to perform the statistical analysis, the more responsive eye was selected. Groups E4-/E4+ and WT-/ WT+ were compared as pairs using one-tailed MannWhitney $U$ test.

\section{Results}

The E4+ group showed statistically significant higher mixed a-wave amplitudes $(P=0.0411)$. In addition, pure cone amplitudes isolated with the double flash protocol also demonstrated statistically significant elevations in amplitude values $(P=0.0260)$ when compared to E4-. No differences were demonstrated in either mixed scotopic or isolated cone implicit times. Standard ISCEV scotopic bright flash b-wave and photopic b-wave amplitudes in the E4+ and WT+ groups resulted in a tendency for higher values in contrast to E4- and WT-, but these values did not reach statistical significance.

\section{Conclusion}

ELOVL4 mice under a DHA-supplemented diet demonstrated protection of retina function (mixed scotopic a-wave and pure scotopic cone b-wave) from one to three months of age. Preservation of amplitude values suggests DHA may potentially have a neuroprotective role on the retina.

\section{Support}

CIHR, AHFMR, CNIB, Lena McLaughlin Foundation (Mona and Rod McLennan), Olive Young. FG: INSERM/CIHR; SCM: FFB Canada. YS: Barbara Tuck/MacPhee Family Vision Research Award in Macular Degeneration.

\section{Poster A13}

\section{Electrophysiological testing-our early experience}

Irene Sophia Joseph, Anupama Rao, B. S. Chidamber

Welcare Eye Center, Welcare World Health Systems, Dubai, UAE

\section{Purpose}

To perform a retrospective study of our outpatients who underwent ERG, EOG and VEP for various ocular conditions, in an attempt to analyse the clinical relevance of Electrophysiological testing to our practice.

\section{Methods}

Our study included 64 cases, 33 women $(51.6 \%)$ and 31 men (48.4\%), tested between June 2006 and February 2008. We performed complete ophthalmological examination with documentation of digital imaging of the fundus. Using the TOMEY EP 1000 electrodiagnostic equipment standard ERG, PERG, EOG and VEP were recorded in accordance with the guidelines of ISCEV, in different combinations according to the perceived need, based on the clinical diagnosis. Each patient's result was compared to our normative data to assess for normalcy of response.

Results

Of the 64 cases, 14 patients had only VEP (either Pattern or Flash) recorded, 18 had only ERG (either standard or pattern), 11 had both VEP and ERG, 12 had both ERG and EOG, and nine had all three tests recorded. There was no case where only EOG or EOG with VEP was performed. Results of 24 patients (43\%) either helped make a clinical diagnosis or clarify a clinical dilemma. Results of eight patients (14\%) helped assess integrity of the visual system when there was unexplained visual loss or media opacity. Results of three patients $(5 \%)$ were unusual for the particular ocular condition. Results of eight patients $(14 \%)$ were not analysed due to inadequate investigations. The tests helped monitor progression of the disease in 21 patients $(37.5 \%)$.

\section{Conclusion}

By including electrophysiological tests in our practice we were able to

(a) test integrity of visual system in cases of unexplained visual loss or inability to assess visual function by other means

(b) get support to confirm or refute the clinical diagnosis of ocular pathologies

(c) provide a clinical diagnostic service to patients and other eyecare practitioners hitherto unavailable in the region.

In conclusion, we feel that electrodiagnostic testing was a valuable tool to complement our outpatient set up in the diagnosis and management of various ocular pathologies.

\section{Poster A14}

Intrasessional and intersessional variability of multifocal electroretinogram

Uzeyir Erdem, Fatih Cakir Gundogan, Gungor Sobaci, Mehmet Zeki Bayraktar 
Gulhane Military Medical Academy, Department of Ophthalmology, Ankara, Turkey

\section{Purpose}

To investigate the intra-sessional and inter-sessional variability of the mfERG.

\section{Methods}

Right eyes of 20 healthy adult subjects with uncorrected visual acuity of 1.0 were studied. MfERG recordings were obtained in two sessions by using jet corneal electrodes. The responses were averaged over five retinal regions, the central hexagon $(\mathrm{CH}$ : $\left.0.0^{\circ}-2.3^{\circ}\right)$ and four concentric rings [ring 1 (R1: $2.3^{\circ}-7.4^{\circ}$ ), ring $2\left(\mathrm{R} 2: 7.4^{\circ}-12.0^{\circ}\right)$, ring $3\left(\mathrm{R} 3: 12.0^{\circ}-19.4^{\circ}\right)$, ring $4(\mathrm{R} 4$ : $\left.19.4^{\circ}-30.0^{\circ}\right)$ ]. In the first session, recordings were repeated while the electrodes stayed in place (the first and the second recordings). Second sessions (third recordings) were performed one week later. Individual coefficient of variation (CV; standard deviation/mean) for P1 and N1 amplitudes and implicit times were calculated for the first to second and first to third recordings to assess intra-sessional and inter-sessional variability, respectively, and were evaluated by Wilcoxon-paired samples test.

\section{Results}

Regarding implicit time and amplitude of mfERG recordings, there was no significant difference between the first to second, and first to third recordings $(P>0.05$, Bonferroni-corrected Wilcoxon test). $\mathrm{CV}$ values significantly decreased with eccentricity in both intra-sessional and inter-sessional comparisons $(P<0.05$, Wilcoxon test). However, partial ring averaging showed no significant difference $(P>0.05)$ between $\mathrm{CV}$ values for single hexagons in each ring. Intra-sessional $\mathrm{CV}$ values were significantly lower than corresponding intersessional values $(P<0.05$, Wilcoxon test $)$.

\section{Conclusions}

Our results support the importance of electrode replacement on variability. The increased number of hexagons that are averaged is the main factor that explains the lower variability in the more peripheral rings.

\section{Poster A15}

\section{Establishment of normal ranges for the ISCEV standard visual evoked potential}

Enid Chelva ${ }^{1}$, Doug Candy ${ }^{1}$, John De Roach ${ }^{1}$, Terri McLaren ${ }^{1}$, Maribel Nosce ${ }^{2}$, Roger Price ${ }^{1}$

${ }^{1}$ Medical Technology and Physics, Sir Charles Gairdner Hospital, Perth, Australia; ${ }^{2}$ Department of Ophthalmology, Sir Charles Gairdner Hospital, Perth, Australia

\section{Purpose}

This study was designed to examine the influence of age and gender on the normal ISCEV standard VEP in an effort to develop meaningful normal ranges for test parameters.

\section{Methods}

The VEP was measured according to ISCEV standards with a check size of $15^{\prime}$ and $60^{\prime}$ for the small and large check stimulus, respectively, both subtending a field size of $16^{\circ}$ at $1.12 \mathrm{~m}$. The standard flash stimulus measured $1.8 \mathrm{~cd} \mathrm{~s} / \mathrm{m}^{2}$. Recordings from 64 normal volunteers, with equal representation of females and closely age-matched males, ranging in age from 3 to 87 with a mean of $41(\mathrm{sd}=21.9)$ years, were made. Results from randomly selected eyes of each subject were used for analysis. Results

Peak implicit times and width were normally distributed for all stimulus types, but this was not the case for peak amplitudes. For the small check VEP, the amplitude of P100 was significantly $(P<0.05)$ larger in females than in males and the $\mathrm{P} 100$ implicit time was the only parameter showing a moderately strong correlation with age $(r=0.63)$. For the large check VEP, the P100 implicit time was weakly correlated with age $(r=0.43)$, with the amplitude also showing a weak, but negative, correlation with age $(r=0.47)$. Flash VEP parameters were found to be highly variable between individuals.

\section{Conclusions}

In this study, VEP implicit times and amplitudes were shown to be affected by age, supporting the use of normal ranges stratified by age. If peak amplitudes are utilised, separate normal ranges should be used for females and males.

\section{Poster A16}

Steady state pattern VEPs: onset-offset checks are more effective than pattern reversal for frequency domain detection

Michelle A. McIntosh ${ }^{1}$, Elena Prokofyeva ${ }^{2}$, Richard G. Boulton ${ }^{3}$, Daphne L. McCulloch ${ }^{1}$

${ }^{1}$ Vision Sciences, Glasgow Caledonian University, Glasgow, Scotland, UK; ${ }^{2}$ Institute of Psychology and Psychoneurology, Northern State Medical University, Arkhangelsk, Russia; ${ }^{3}$ Department of Clinical Physics and Bioengineering, Princess Royal Maternity and University of Glasgow, Glasgow, Scotland, UK

\section{Purpose}

Steady-state VEPs (SS-VEPs) are used in applications that require rapid, quantitative signal detection. SS-VEP signals vary with presentation mode and with temporal presentation rate. We compare two modes of pattern presentation: pattern reversal (PR) and pattern onset-offset (ON-OFF) across a range of presentation rates.

\section{Methods}

Participants were 10 healthy adult volunteers (aged 18-25 years), with no visual or ocular motor dysfunction. Pattern stimuli were high-contrast, ISCEV-standard, small checks $\left(0.25^{\circ}\right.$ check width) presented in PR and ON-OFF modes. Five temporal rates between 3.42 and 18.6 were used. PR checks were presented at the reversal rate and ON-OFF stimuli were presented at the cycle frequencies $(50 \%$ duty cycle alternated with luminance-matched grey). In addition, fullfield, square-wave modulated flicker $(50 \%$ duty cycle, $200 \mathrm{~cd} /$ $\mathrm{m}^{2}$ ) stimuli were presented at the same rates. Signals were averaged for an integer number of cycles using total periods of $40 \mathrm{~s}$ or less. The magnitude and signal-to-noise ratio (SNR) of Fourier components of the SS-VEPs were compared at the fundamental stimulation rates (F1) and at double these rates (F2) using ANOVA with Bonferroni corrections.

\section{Results}

Overall, ON-OFF presentation of checks produced larger VEPs and SNRs than did PR at both F1 and F2 $(P<0.01)$. For both 
presentation modes SS-VEP signals were strongest for temporal rates between 7.14 and 12.7 (reversals/s or $\mathrm{Hz}$ for PR and $\mathrm{ON}-\mathrm{OFF}$, respectively) probability of detection of F1, F2 or both. However, the frequency response functions differed between the presentation modes; at both F1 and F2 SS-VEPs for ON-OFF checks followed a high pass function with similar magnitude and SNR values for frequencies up to $12.5 \mathrm{~Hz}$. For PR checks the SS-VEPs were band pass with significantly low magnitude and SNRs values at the high and low reversal rates. Differences between $\mathrm{ON}-\mathrm{OFF}$ and PR were significant for stimulation rates of 3.4 for $\mathrm{F} 1$ and $\mathrm{F} 2$ and at $4.6 \mathrm{~Hz}$ for $\mathrm{F} 2$. Interestingly, the full field $\mathrm{ON}-\mathrm{OFF}$ luminance flicker produces SS-VEPs with a band-pass frequency response function similar to that of the PR checks with undetectable signals at low and high stimulation rates

\section{Conclusions}

SS-VEPs to luminance flicker and to PR checks are poorly detected for presentation rates below 5 and above $15 \mathrm{~Hz}$. In contrast, checks presented in the ON-OFF mode elicit strong SS-VEPs for stimulation frequencies as low as $3.4 \mathrm{~Hz}$. Using relatively short presentation times, SS-VEPs in young adults are strongest when presented at $7-13 \mathrm{~Hz}$ with signal detection at F1 and F2.

\section{Support}

Individual Mobility Grant RF3006-2006 from European Commission awarded to Elena Prokofyeva.

\section{Poster A17}

\section{Stroboscopic and square wave flicker VEPs for visual pathways assessment}

Elena Prokofyeva ${ }^{1}$, Michelle McIntosh $^{2}$, Richard G. Boulton ${ }^{3}$, Daphne L. McCulloch ${ }^{2}$

${ }^{1}$ Institute of Psychology and Psychoneurology, Northern State Medical University, Arkhangelsk, Russia; ${ }^{2}$ Vision Sciences, Glasgow Caledonian University, Glasgow, Scotland, UK; ${ }^{3}$ Department of Clinical Physics and Bioengineering, Princess Royal Maternity and University of Glasgow, Glasgow, Scotland

\section{Purpose}

Steady-state VEPs (SS-VEPs) to luminance flicker are useful for evaluation of visual pathways, particularly for those who have poor fixation or limited cooperation. SS-VEP waveforms are influenced by the luminance profile of the flicker. We compare stroboscopic and square-wave flicker across a range of stimulus frequencies to compare the normal frequency response characteristics for SS-VEPs to these common modes of flicker presentation.

\section{Methods}

Participants were 10 healthy adult volunteers (aged 18-25 years), with no visual or ocular motor dysfunction and corrected visual acuity of 6/6 (0.0 Log MAR) or better in each eye. SS-VEPs were recorded binocularly to full field flicker stimuli generated using LEDs in a ganzfeld (Espion Colordome ${ }^{\circledR}$ ). Two modes of flicker were presented: square-wave modulated (ON-OFF with $50 \%$ duty cycle, $200 \mathrm{~cd} / \mathrm{m}^{2}$ ) and stroboscopic flash $\left(5 \mathrm{~ms}\right.$ pulses, $\left.10 \mathrm{~cd} / \mathrm{m}^{2} / \mathrm{s}\right)$ presented at six temporal frequencies: $3.42,4.64,7.32,12.7,18.6$ and $38.1 \mathrm{~Hz}$. The magnitude of Fourier components of SS-VEPs at the fundamental stimulus frequencies (F1) and double harmonics (F2) and the signal-to-noise ratios (SNR) were compared using ANOVA with Bonferroni corrections.

\section{Results}

For both modes of stimulation, flicker at 7.3 and at $12.7 \mathrm{~Hz}$ elicited a significant SS-VEP in all participants $(P<0.05)$, usually for both at F1 and F2. Using these recording parameters, the SS-VEP was also detectable in $9 / 10$ subjects at 4.6 and $18.6 \mathrm{~Hz}$ but poorly detected for the slowest $(3.4 \mathrm{~Hz})$ and fastest $(38 \mathrm{~Hz})$ flicker rates. SS-VEP magnitudes are larger for F1 than for F2 $(P<0.001)$. Square-wave modulated flicker gave higher SNR values at F1 than those for stroboscopic flicker $(P=0.001)$; this difference was significant individually for 12.6 and $18.6 \mathrm{~Hz}$ flicker (post hoc tests, $P<0.01$ ). F2 harmonics were best detected from 4.6 to $12.7 \mathrm{~Hz}$ but did not differ between the modes of stimulation.

\section{Conclusions}

Flickering stimuli elicit reliable SS-VEPs in young adults using flicker rates between 7 and $19 \mathrm{~Hz}$. Square-wave modulated flicker has an advantage over stroboscopic flicker as it elicits higher SNRs. These parameters can be used to assess the visual pathways of patients with poor fixation.

\section{Support}

Individual Mobility Grant RF3006-2006 from European Commission awarded to Elena Prokofyeva.

\section{Poster A18}

\section{Effects of anisometropia on binocular integration} in normal observers

L. Lefebvre ${ }^{1,2}$, D. Saint-Amour ${ }^{1,2,3}$

${ }^{1}$ Centre de Recherche, CHU Sainte-Justine; ${ }^{2}$ Centre de Recherche en Neuropsychologie et Cognition, Département de Psychologie de l'Université de Montréal; ${ }^{3}$ Département d'ophtalmologie de l'Université de Montréal

\section{Purpose}

The neurophysiological mechanisms underlying anisometropic amblyopia in humans remain unknown. To better understand how one eye can suppress the other eye under anisometropic conditions, pattern-reversal VEP was examined in eight normal observers for whom artificial anisometropia (moderate myopia) was induced by converging lenses.

\section{Methods}

Reversal checkerboards (four reversals/s) were presented at a contrast of $96 \%$ and a spatial frequency of 2 cpd according to three conditions: no refractive error, refractive error $(+3.25 \mathrm{D})$ in both eyes, and refractive error $(+3.25 \mathrm{D})$ in one eye. VEP was recorded over the occipital region from Oz. Indexes of binocular integration (binocular response subtracted from the sum of monocular responses) were computed from the P100 latency and the N75-P100 peak-to-peak amplitude and analyzed with repeated measures ANOVAs.

\section{Results}

As expected for latency, the comparison of monocular and binocular VEP revealed significant differences for normal binocular viewing as well as for binocular refractive error viewing. In both cases, these effects indicated significant 
binocular facilitation (the binocular response was faster than each of the monocular response). By contrast, binocular integration disappeared in the anisometropic condition when the refractive error was applied to only one eye $(P>0.05)$. Moreover, this effect showed evidence of binocular suppression, i.e., the binocular response was driven by the best eye response. VEP amplitude analysis revealed a significant difference of binocular integration between the binocular normal viewing condition and the anisometropic condition $(P<0.05)$. However, no significant difference was found between the binocular refractive error condition and the anisometropic condition $(P>0.05)$. This unexpected result could indicate that different integrative mechanisms are involved when refraction is applied to both eyes due to the fact that the brain of normal observers is not used to such a situation.

\section{Conclusion}

Although VEP latency is a robust measure to reveal binocular interactions under anisometropic viewing, VEP amplitude should also be taken into account because of its potential to reveal new elements about the mechanisms underlying interocular suppression. This study supports the notion that the binocular deficit observed in anisometropic amblyopes is associated with a suppression of the emmetropic eye over the ametropic eye.

\section{Poster A19}

\section{A comparison of visual acuity estimates from visual evoked cortical potentials, fract and ETDRS}

\author{
A. Kurtenbach ${ }^{1}$, H. Langrová ${ }^{2}$, A. Messias ${ }^{1}$, E. Zrenner ${ }^{1}$, \\ H. Jägle ${ }^{1}$ \\ ${ }^{1}$ Centre for Ophthalmology, Tübingen, Germany; ${ }^{2}$ Institute \\ of Ophthalmology, Hradec Králové, Czech Republic
}

\section{Purpose}

The aim of this study was to compare the visual acuities, as estimated from Visual Evoked Cortical Potentials (VEPs), obtained by two different methods of recording and two different electrode placements. These results were additionally compared to those obtained by two subjective measures of visual acuity.

\section{Methods}

Ten subjects, 4 males, 6 females, (26-67 years) were examined. Transient sweep VEPs (tVEP), based on the method of Hajek and Zrenner (1988), and steady-state VEPs (ssVEP), adapted from Wolf and Bach (2006), were each recorded twice and with two different electrode placements (10-20 or Laplace). Subjective measurements of visual acuity were ascertained using the Freiburg Visual Acuity Test (FrACT) and the ETDRS chart. Visual acuities were measured by all four methods with subjective best optical correction and induced blur using Bangerter occlusion foils, which reduced visual acuity to ca. 0.8, 0.6, 0.3, 0.2 and 0.1 (decimal scale).

\section{Results}

Comparison of the log MAR visual acuities measured by FrACT with those measured by ETDRS gave a mean difference of 0.11 with limits of agreement of -0.14 to 0.35 .
The mean difference between FrACT and tVEP was -0.12 , with limits of agreement between -0.418 and 0.185 . The Laplace electrode placement did not improve tVEP analysis. While VEP response amplitudes were higher for tVEPs, a more objective analysis could be performed for the ssVEPs by calculating signal-to-noise ratios.

\section{Conclusions}

VEP estimates are generally beset by a significant variability and reduced repeatability, allowing only rough individual acuity estimates from VEP measurements. Nevertheless, if VEP responses show good signal-to-noise ratio, they can be used to estimate the visual acuity.

\section{Support}

German Research Foundation grant JA997/8-1 awarded to HJ and EZ and the Tistou and Charlotte Kerstan Foundation Vision 2000 .

\section{Poster A20}

\section{VEP asymmetry versus chiasm coefficient in children with MRI confirmed achiasmia}

Jelka Brecelj ${ }^{1}$, Maja Šuštar ${ }^{1}$, Branka Stirn-Kranjc ${ }^{1}$, Nuška Pečarič-Meglič ${ }^{2}$, Miha Škrbec ${ }^{2}$

${ }^{1}$ Eye Clinic and ${ }^{2}$ Clinical Institute for Radiology, University Medical Centre, Ljubljana, Slovenia

\section{Purpose}

VEPs can distinguish normal decussation of the optic nerve fibres at the optic chiasm from congenitally reduced decussation in achiasmia. This study examined the crossing of the optic nerve fibres at the chiasm according to the occipital distribution of flash VEP (N2, P2 waves) and the chiasm coefficient.

\section{Method}

Four children (7-12 years) with achiasmia (two also with hypoplastic right optic tracts) and 11 healthy children (4-6 years) were included in the study. The VEP to monocular flash stimulation were recorded from three occipital electrodes. The chiasm coefficient was calculated from the differential inter-hemispheric signals between 0 and $285 \mathrm{~ms}$.

\section{Results}

The occipital distribution of the N2 and P2 waves differed: in the healthy children they were more symmetrical over both hemispheres, while in the children with achiasmia they were asymmetrical; in two children over the ipsilateral hemisphere to the stimulated eye and in other two children (plus hypoplastic right optic tracts) the asymmetry was in accordance to right optic tract maldevelopment. The chiasm coefficients showed differences between the healthy children ( -0.49 to 0.71 ) and $2 / 4$ with achiasmia (in $2 / 4$ : -0.85 and $-0.74)$. In the other two children with achiasmia plus hypoplastic right optic tracts, the chiasm coefficients were positive (0.63 and 0.90) and were not different from those of the healthy children.

\section{Conclusions}

When distinguishing normal from abnormal chiasmal decussation, both the chiasm coefficient and the VEP occipital distribution are of value. 


\section{Oral Session 3: Low vision and retinal function}

\section{Chairs: William Seiple \& Sandeep Grover}

\section{Invited Lecture 2: Rehabilitation in visually impaired children: "coming to better terms...with"}

\section{Frans C. C. Riemslag}

Bartiméus Institute for the Visually Impaired, Zeist, The Netherlands

For a youngster to come to better terms with his or her visual disorder, it is imperative to understand his or her visual functioning. In our daily practice in the (electro)ophthalmology department of Bartiméus, a Dutch institute for the rehabilitation of the visually impaired, our primary aim is to establish the diagnosis in the children referred to our care in detail. This provides information about the prognosis of their participation possibilities, including expectations about the need for care as well as the basis for informed genetic counseling.

To maximize the diagnostic value of electrophysiological testing we use an extension of the standard ISCEV protocols for both the ERG and the VEP. After establishing the diagnosis we evaluate limitations for instance caused by reduced visual acuity (e.g. in "functional" vision loss), "night blindness" (e.g. CSNB, RP) and the "blinding" aspects of high luminance levels (e.g. achromatopsia, enhanced S-cone syndrome and albinism). This allows for tailor made adaptations to the participation restrictions at hand.

An overview of 3 years' practice presented to ISCEV in Glasgow showed that, as a result of our electrophysiological assessment, in a certain percentage $(10 \%)$ the diagnosis (with which the child was referred to us) had to be changed from a progressive into a stationary disorder or the reverse. It is obvious that these parameters drastically change the strategy to attain "coming to terms with the disorder".

Having thus established a diagnosis in detail, it turns out that for the above mentioned youngster, for the parents of a visually impaired child and for the professionals in the rehabilitation institutes, the terminology used to describe the disorder can be unnecessarily alarming rather than comprehensible or even realistic. In this keynote it will be concluded that quite a number of terms used do not at all contribute to a better understanding, quite the opposite one should say.

\section{OS3-Oral Paper 1}

To assess the functional activity of the outer retinal layers at the pseudofovea in eccentric fixation

Ajoy Vincent ${ }^{1}$, Rohit Shetty ${ }^{1}$, Vasudha Naresh ${ }^{2}$, M. Bhanumathi ${ }^{2}$, Bhujang Shetty ${ }^{1}$

${ }^{1}$ Department of Electrophysiology and ${ }^{2}$ Department of Pediatric Ophthalmology, Narayana Nethralaya, Super Specialty Eye Hospital and Post Graduate Institute, Bangalore, India

\section{Purpose}

To analyse the cone photoreceptor and cone bipolar cell functional activity at the pseudofovea, in subjects with eccentric fixation accompanying strabismic amblyopia.

\section{Methods}

Prospective, non-randomized, cross-sectional, case control study was undertaken after Institutional ethical committee clearance. Ten eyes of 10 subjects with strabismic amblyopia with eccentric fixation underwent ophthalmologic evaluation including bestcorrected visual acuity, angle of ocular deviation, fundus photography and multifocal electroretinography (mfERG). To obtain the responses, a 36-block non-scaled mfERG stimulus was used. A binary sequence of 512 D2 was used, and 3,500 stimuli were averaged. Each testing run lasted for $3 \mathrm{~min}$ and $30 \mathrm{~s}$. The mean luminance of the CRT was $200 \mathrm{~cd} / \mathrm{m}^{2}$ and the contrast was $95 \%$. A uniformly illuminated background cover of $30 \mathrm{~cd} / \mathrm{m}^{2}$ surrounded the stimulus screen. The high and low band filters were set at 0.1 and $72 \mathrm{~Hz}$, respectively. The mfERG recording from the other eye of the subjects served as the control. Fundus photograph was obtained with the subject looking straight ahead at the central fixation spot of the fundus camera. The N1 and P1 amplitudes of the response obtained from the zone of pseudofovea, zone surrounding pseudofovea, zone of true fovea in eccentrically fixing eye (in same angle eccentric fixation) and the zone of the fovea in the centrally fixing eye were compared. Results

The N1 and P1 amplitudes of mfERG recording from the zone of eccentric fixation were higher than the surrounding zones in seven eyes. The N1 and P1 amplitudes from the zone of eccentric fixation were significantly low when compared to the $\mathrm{N} 1$ and P1 amplitudes from the zone of the fovea in the other eye in eight subjects. The eccentric fixation was para foveal in two eyes and hence the zones used for stimulation of eccentric fixation and true fovea overlapped in these eyes. The N1 and P1 amplitudes from the true foveal zones of the two other smaller angled eccentrically fixated eyes were significantly higher than the N1 and P1 amplitudes obtained from the zone of eccentric fixation. The N1 and P1 amplitudes from the zones of the true fovea in eccentrically fixating eyes of four subjects were reduced but comparable to the $\mathrm{N} 1$ and $\mathrm{P} 1$ amplitudes obtained from the zone of the fovea in the other eye.

\section{Conclusion}

There is enhancement of outer retinal activity at the pseudofovea in most cases of eccentric fixation. The higher amplitude responses from zones of eccentric fixation functionally suggest that pleoptics may be used to enhance vision in such eyes in older age group subjects. Further, the near normal amplitudes of N1 and P1 from the zones of the true fovea in eccentrically fixating eye suggest that intensive occlusion therapy should be done in younger children to encourage improvement in both vision and fixation in such subjects.

\section{OS3-Oral Paper 2}

Microcurrent neuromodulation in the management of dry age-related macular degeneration

Sundaram Natarajan, Mahesh Uparkar, Anjali Khulsange, Darshana Gadgil

Aditya Jyot Eye Hospital Pvt Ltd, Wadala, Mumbai-31, India

Purpose

To describe the short-term outcome of a pilot study to evaluate safety and efficacy (clinical and electrophysiological) of 
microcurrent neuromodulation (MCN) in dry age-related macular degeneration (AMD).

\section{Methods}

In a prospective, consecutive case-series in a single center in Mumbai, India, patients older than 50 years with dry macular degeneration with visual acuity $<20 / 30$ but $>20 / 200$ were recruited. Dry AMD was defined as the presence of soft drusen ( $>63$ microns), hyperpigmentation at macula, and/or geographic atrophy in the macular area and was classified as early $(<5$ soft drusen plus hyper-pigmentation-stage I), intermediate $(>5$ soft drusen-stage II), and late (any geographic atrophy-stage III). Patients with cataract, optic nerve, or retina diseases or prior retinal surgeries were excluded from the study. MCN of the eyes was done as per protocol algorithm twice daily for 20 min during the period of study using ScyFIX $650^{\mathrm{TM}} \mathrm{MCN}$ device. Patients with intermediate stage disease were given anti-oxidant supplementation along with MCN. Protocol examinations included visual acuity (log MAR) with refraction, complete ophthalmic evaluation, OCT (Stratus, Carl Zeiss), fluorescein angiography, multifocal ERG (mfERG-Veris), contrast sensitivity (FACT), color vision (Ishihara), and VFQ-25 (NEI). This was done at baseline and then at 1, 3 and 6 months. All adverse events were noted during the period of study. Study protocol was approved by Institutional Ethics committee and adhered to the tenets of the declaration of Helsinki. Statistical analysis was done using paired-t-test for parametric data (SPSS Inc, IL, USA).

\section{Results}

Seventeen patients (33 eyes) were included in the study. Mean age was 66.7 years (range 56-78 years; 11 males). Stage I disease was seen in 8 eyes, 9 eyes had Stage II, and 16 eyes had Stage III. Baseline mean log MAR visual acuity was $0.37 \pm 0.03$ in Stage I, $0.39 \pm 0.18$ in Stage II, and $0.70 \pm 0.30$ in Stage III eyes. At 6-month follow-up, this changed to $\log$ MAR $0.15 \pm 0.03(P=0.031), 0.30 \pm 0.09$ $(P=0.128)$, and $0.55 \pm 0.23(P=0.035)$ in Stages I, II and III, respectively. At 6 months, contrast sensitivity $(n=19)$ in each stage of the disease showed a mean improvement of $>1$ grating acuity at high $(1.5 \mathrm{cpd})$ and medium $(3 \mathrm{cpd})$ contrast grating-acuity, while the low contrast grating acuity did not change. Among 4 eyes with confluent soft drusen, two showed a reduction in size and number on OCT while the other two remained the same at last follow-up. Mean response density (P1-N1) change in the central $5^{\circ}$ of the retina (kernel 1-ring 1) in each stage of the disease at 6 months was analyzed. Stage I eyes showed a change from $12.6 \pm 1.8$ to $35.5 \pm 3.1 \mathrm{nv} / \mathrm{deg}^{2}(P=0.022)$. In stage II eyes, the response density changed from $38.5 \pm 2.9$ to $43.5 \pm 5.3 \mathrm{nv} / \mathrm{deg}^{2} \quad(P=0.046)$. In Stage III eyes, the response density changed from $36.0 \pm 2.3$ to $31.2 \pm 3.4$ nv/deg ${ }^{2}(P=0.082)$. No significant adverse events were noted during the first 6 months of study.

\section{Conclusion}

$\mathrm{MCN}$ of the eyes in various stages of dry macular degeneration was safely administered and demonstrated significant benefit with regard to visual acuity, contrast sensitivity and mfERG changes in the short term. Long-term follow-up with randomized controlled study is necessary to validate its extended benefit.

\section{OS3-Oral Paper 3}

\section{Delayed implicit time of pattern visual evoked potentials in patients with occult macular dystrophy}

Kazuha Ogata, Shuichi Yamamoto

Department of Ophthalmology and Visual Science, Chiba University Graduate School of Medicine, Chiba, Japan

\section{Purpose}

To investigate pattern VEPs in patients with occult macular dystrophy (OMD).

\section{Methods}

Two unrelated patients, a 64-year-old man (Case 1) and a 38-year-old man (Case 2), clinically and electrophysiologically diagnosed as OMD, were examined. Both had normal ocular fundus, normal fluorescein angiography, and normal full-field ERGs but reduced responses from macular area in mfERGs. VEPs were recorded to black and white pattern stimuli.

\section{Results}

The implicit times of P100 of the VEP's were prolonged to $142 \mathrm{~ms}$ in the right eye and $142 \mathrm{~ms}$ in the left eye in Case 1, and $126 \mathrm{~ms}$ in the right eye and $126 \mathrm{~ms}$ in the left eye in Case 2 (normal limits $<120 \mathrm{~ms}$ ). The amplitudes of P100 were reduced but within normal limits in both patients.

\section{Conclusions}

Delays in the implicit times of VEP are usually indicative of optic nerve diseases, and may lead to misdiagnosis. Actually, Case 2 was diagnosed as optic neuritis initially and treated with systemic corticosteroid by previous ophthalmologists. Our results indicate that ophthalmologists should be careful in deciding on diagnosis between OMD and optic nerve disease.

\section{OS3-Oral Paper 4}

\section{A causal role for the rods in retinopathy of prematurity}

James D. Akula ${ }^{1,2}$, Ronald M. Hansen ${ }^{1,2}$, Tara L. Favazza ${ }^{1}$, Tanya C. Vyhovsky ${ }^{1}$, Ilan Y. Benador ${ }^{1}$, Julie A. Mocko ${ }^{1}$, Anne B. Fulton ${ }^{1,2}$

${ }^{1}$ Department of Ophthalmology, Children's Hospital Boston, MA; ${ }^{2}$ Harvard Medical School, Boston, MA

\section{Purpose}

Although retinopathy of prematurity (ROP) is clinically characterized by tortuous retinal vasculature, the neural retina is also rendered significantly-and persistently-dysfunctional. In rat models of ROP, we recently demonstrated that early low rod sensitivity predicts later high vascular tortuosity. The "temporal priority" in rod dysfunction supports a causal role for the rods in ROP. However, experimental manipulation of rod function is necessary to confirm a causal role for the rods. In the present experiments, we seek to clarify the role of the neural retina in ROP, with the goal of improving the management of ROP.

\section{Methods}

We induce ROP in rats by exposure to relatively high and low oxygen during the critical period of rod outer segment elongation as previously described. This induces a range of 
neurovascular outcomes that mirror the broad spectrum of human ROP. Rod photoreceptor (SROD) and postreceptor $(\log \sigma)$ sensitivity are derived from the respective ERG a- and b-waves. Blood vessel tortuosity is quantified from digital fundus photographs (integrated curvature of the retinal vessels, IC). We are conducting two parallel studies: (1) Animals undergo ERG testing and fundus imaging and are then sacrificed. We extract their retinae and assay mRNA expression of three growth factors that cooperatively control both neuro- and angiogenesis, vascular endothelial growth factor (VEGF), semaphorin 3A (SEMA 3A), and their neuropilin receptor (NRP 1). (2) In a second group of rats, we inject a novel vitamin-A derivative during the pathogenesis of ROP to modulate rod photoreceptor function. Effects on the ERG and the retinal vasculature are longitudinally assessed.

\section{Results}

As in human patients, ROP rats' vascular tortuosity is high at young ages, especially in the arterioles (ICA), and resolves as the eye matures. The resolution of ICA goes hand-in-hand with improvements in SROD and $\log \sigma$. Data from our molecular biological experiments support a role for the rods in ROP: Immediately after induction, VEGF is highly correlated with ICA $(r=0.68 ; P=0.001)$, and SROD is highly correlated with VEGF $(r=0.75 ; P<0.001)$ as well as ICA $(r=0.54$; $P=0.004)$. Pharmacological alteration of rod function in the immature ROP rat retina increases the time constant of deactivation of phototransduction $(\tau)$ and results in increased resolution of ICA in treated rats $(\mathrm{F}=11.0 ; \mathrm{df}=44$; $P=0.002$ ).

\section{Conclusions}

Our data support a causal role for the neural retina in ROP, especially for the rod photoreceptors. It now appears likely that ROP results primarily in damage to developing retinal neurons, and secondarily in the abnormal retinal vasculature. The vascular abnormalities may be mediated by molecular pathways at least partially under control of the rods. If true, treatment of the neural retina is an essential element of any pharmaceutical intervention in ROP.

\section{Oral Session 4: Evaluation of toxicity and visual function}

\section{Chairs: Daphne McCulloch \& Mineo Kondo}

\section{OS4-Oral Paper 1}

ERGs during examination-under-anesthesia: responses of normal fellow eyes

Scott E. Brodie ${ }^{1,2,3}$, Dawn Desiderio ${ }^{1,2}$, David H. Abramson ${ }^{1,2}$

${ }^{1}$ Memorial Sloan-Kettering Cancer Center, New York, NY, USA; ${ }^{2}$ Weill-Cornell Medical College, New York, NY, USA; ${ }^{3}$ Mount Sinai School of Medicine, New York, NY, USA

\section{Purpose}

To summarize the ERG response parameters of clinically normal fellow eyes of patients undergoing examinationunder-anesthesia for unilateral eye disease, which may help provide normative data for electroretinography under anesthesia.

\section{Methods}

Retinal function of patients undergoing examination-underanesthesia ("EUA") for unilateral eye disease (mostly after selective ophthalmic artery chemotherapy infusion for retinoblastoma) was monitored by serial ERG studies. Inhalation anesthesia (Sevoflurane) was induced by mask and maintained with an LMA airway. Ganzfeld ERGs were obtained from each eye separately using an ERG-jet contact lens electrode. The stimulus was provided with a hand-held monocular miniganzfeld stimulator (Diagnosys ColorBurst). Recording adhered to the ISCEV standard protocol except that scotopic responses were measured after only 5 min of dark adaptation in order to reduce the total duration of anesthesia.

\section{Results}

Compared with conventional recording, ERG baselines during EUA were somewhat unsteady. Photopic responses to single flash and $30-\mathrm{Hz}$ flicker were slightly smaller than under standard conditions, but photopic B-wave and flicker implicit times and latencies were usually within traditional normal limits, or only slightly prolonged. The scotopic response to the full-strength ISCEV Standard Flash rarely reached the normal amplitude seen under standard conditions. Variability was increased.

\section{Conclusions}

Interpretation of ERGs obtained at EUA should allow for greater variability than seen under standard conditions. Response amplitudes may be somewhat reduced, especially under scotopic conditions.

\section{OS4-Oral Paper 2}

\section{Postnatal exposure to bright light stimulates retinal plasticity}

P. Lachapelle, A. Polosa, E. Zimak, W. Liu, L. Robbe,

S. Joly, S. G. Rosolen, S. Chemtob

Ophthalmology and Neurology-Neurosurgery, McGill

University/Montreal Children's Hospital, Montreal, PQ,

Canada

Purpose

Compare the structural and functional consequences of postnatal Light-Induced Retinopathy (LIR) in juvenile albino and pigmented rats.

\section{Methods}

Juvenile Sprague Dawley (SD) and Long Evans (LE) rats exposed from P14-20 and P14-28 to a bright light (12D: 12L; 10,000 lux) were compared to adult rats exposed to the same light for 6 days. Photopic (intensity: $0.9 \mathrm{log} \mathrm{cd} \mathrm{s} / \mathrm{m}^{2}$; background: $30 \mathrm{~cd} / \mathrm{m}^{2}$ ) flash ERGs (fERG), flash VEPs (fVEP) and multifocal ERGs (mfERG: VERIS 5.1; camera display unit, 37 hexagons; white: 200; black: 0; background: $100 \mathrm{~cd} / \mathrm{m}^{2}$; bandwidth: $10-100 \mathrm{~Hz}$ ) were recorded at regular intervals between P30 and P60.

Results

fERGs: In SD rats, ERG responses (P14-P20 and P14-P28 groups) at $\mathrm{P} 30$ were significantly reduced in amplitude $(P<0.05)$ compared to the normal cohort. Between P30 and 
P35, the fERG amplitudes increased in the P14-P28 group while it decreased in the P14-P20 group as well as in normal rats. At P60, ERG amplitudes were still significantly smaller than normal in the two exposed groups $(P<0.05)$, but significantly larger than in adult LIR. Similar findings were also observed at P30 and between P30 and P35 in LE rats. However, at P60, the ERGs were significantly larger $(P<0.05)$ than normal in the exposed LE group. Histology also revealed thicker than normal retinas in exposed LE rats. fVEP: At P30, the typical fVER is composed of two major positive components peaking at approximately 50 and $100 \mathrm{~ms}$ after the flash. Light exposure significantly attenuated the short latency component and slightly enhanced the long latency one. At P60, control and exposed rats lacked the short latency component while the long latency one remained larger than normal. In adult LIR the fVEP was reduced to $50 \%$ of normal. mfERG: In control rats, the mfERG superior/inferior retina ratios were $1.02 \pm 0.11$ and $1.03 \pm 0.11$ at $\mathrm{P} 30$ and $\mathrm{P} 60$, respectively, while in juvenile LIR it was within the normal range $(P<0.05)$ at $\mathrm{P} 30$ and significantly reduced $(0.84 \pm 0.23 ; P<0.01)$ at $\mathrm{P} 60$ compared to $0.71 \pm 0.01$ $(! P<0.01)$ in adult LIR. While at P30 retinal section revealed minimal hemiretinal differences in juvenile LIR, at P60 the superior retina was significantly thinner than the inferior retina. In adult LIR the superior retina was nearly destroyed.

\section{Conclusions}

1. The fERG enhancement observed between P30 and P35 in the P14-P28 exposed group suggests a photostasis effect. 2. Relative preservation of the fVEP response is most probably the consequence of the significantly thicker Inner Nuclear Layer (INL) observed in retinas of exposed rats. A thicker INL would amplify the weaker signal from the thinner ONL (due to light exposure) in order to generate a near normal retinal output. 3. Results obtained in LE rats would suggest reduction of apoptosis by postnatal light exposure. It remains to be determined if intense light exposure could trigger similar compensatory processes in diseased retina.

\section{Support}

CIHR and Réseau Vision.

\section{OS4-Oral Paper 3}

\section{Long-term methamphetamine abuse influences visual evoked potentials}

Jan Kremláček ${ }^{1}$, Miroslav Kuba ${ }^{1}$, Ladislav Hosák ${ }^{2}$, Jan Libiger ${ }^{2}$, Jiří Č́žzek ${ }^{2}$

${ }^{1}$ Department of Pathological Physiology, Charles University in Prague, Faculty of Medicine in Hradec Králové, Czech Republic; ${ }^{2}$ Department of Psychiatry, Charles University in Prague, Faculty of Medicine in Hradec Králové and University Hospital Hradec Králové, Czech Republic

\section{Purpose}

Methamphetamine (MA) is a widespread psycho-stimulant substance of abuse that affects brain dopamine, noradrenalin and serotonin extracellular levels. The long-term use of the MA is neurotoxic and leads to damage of dopamine terminals. We investigated whether MA neurotoxicity can impair the visual system by studying the VEPs in MA dependent individuals.

\section{Methods}

We recorded pattern-reversal (P-VEPs) and motion-onset (M-VEPs) VEPs in a group of 23 recently abstaining MA abusers ( 5 females, MA abuse $5.3 \pm 2.8$ years) and the ageand sex-matched group of healthy individuals

Results

We found prolonged latencies $(P<0.01)$ of the P100 peak of P-VEPs to 10' and 20' checkerboard stimuli and the N2 peak of M-VEPs to radial motion-onset in full stimulus field $\left(28^{\circ} \times 37^{\circ}\right)$ or in central $8^{\circ}$ among former MA abusers. The P-VEPs to $40^{\prime}$ pattern-reversal and the M-VEPs to peripheral radial motion-onset (outside the central $20^{\circ}$ ) or to translation motion-onset did not differ significantly between groups.

\section{Conclusions}

The prolonged VEPs among subjects following long-term methamphetamine abuse agree with the effect of methamphetamine neurotoxicity on visual information processing.

\section{Support}

Ministry of Education of the Czech Republic (VZ 0021620820 and VZ 0021620816).

\section{OS4-Oral Paper 4}

\section{ERG effects of chronic (daily) sildenafil or tadalafil usage}

Michael F. Marmor ${ }^{1}$, Christopher Zoumalan ${ }^{1}$, Roham T. Zamanian ${ }^{2}$, Ramona L. Doyle ${ }^{2}$, William H. Cordell ${ }^{3}$

${ }^{1}$ Department of Ophthalmology, Stanford University, Stanford, CA; ${ }^{2}$ Division of Pulmonary and Critical Care Medicine, Stanford University, Stanford, CA;

${ }^{3}$ Lilly Research Labs, Eli Lilly Co. Indianapolis, IN

\section{Purpose}

Sildenafil can cause transient, mild ERG changes in healthy individuals taking large single doses (e.g., 100-200 mg). Although erectile dysfunction (ED) drugs were originally intended for intermittent use, they are now being used continuously for patients with pulmonary arterial hypertension (PAH) and other conditions, sometimes in very high doses (e.g., 50-75 mg 3 to 4 times a day). We investigate possible ERG changes in patients using ED drugs on a chronic basis.

\section{Methods}

Four Stanford patients with PAH (age $40 \pm 5.2$ years) who had used high-dose sildenafil $(225 \pm 50 \mathrm{mg}$ daily $)$ for 2-4 years were studied with color vision (standard and desaturated Panel D-15 test), full-field ERG (UTAS E-2000 system), and multifocal ERG (mfERG, VERIS system). Two of these also had mfERG testing while off medication for 9-12 h. A separate group of 244 patients in an Eli Lilly Co. study were randomized to low-dose tadalafil $5 \mathrm{mg}$, sildenafil $50 \mathrm{mg}$, or placebo daily. ISCEV standard ERGs (Espion system) and color vision (Farnsworth 100-Hue test) were evaluated.

\section{Results}

The high-dose patients had 20/20 visual acuity and showed color confusions only on the desaturated Panel D-15 test. Fullfield ERGs showed normal dark-adapted responses; cone 
amplitudes were normal in 3 of 4 subjects, but all 4 showed borderline or elevated cone implicit time to single flash and $30 \mathrm{~Hz}$ stimulation. The mfERG responses were of normal amplitude, but delayed in 3 of 4 subjects. The two patients who transiently stopped medication showed a small increase in mfERG amplitude and decrease in implicit time (average $1 \mathrm{~ms}$ ) that returned to baseline 1 hour after taking the medication again. The low-dose patients showed no significant differences between treatment groups in any full-field ERG parameters (including cone implicit times) or in 100-Hue color sensitivity. Conclusions

Our results for high-dose chronic sildenafil show a lengthening of cone implicit time that is comparable to effects reported with large (100-200 mg), single doses. There is a suggestion that some of these changes may be reversible in the short term but further studies will be needed to assess whether there are any permanent ERG effects. The low-dose study on chronic sildenafil and tadalafil showed no effects of either agent relative to placebo controls. Chronic ED drug usage, even up to these higher dosage levels, does not appear to be seriously toxic or threatening to vision.

Financial disclosures: Dr. Marmor is a consultant for Pfizer and Lilly; Dr. Cordell is an employee of Lilly.

\section{OS4-Oral Paper 5}

\section{Is it possible to use the mfERG to follow-up hydroxychloroquine therapy in elder patients?}

I. Ingster-Moati $^{1,2,3}$, E. Bui Quoc ${ }^{2}$, C. Orssaud ${ }^{2}$, D. Schmitt ${ }^{2}$, Ph. Le Neindre ${ }^{2}$, J. L. Dufier ${ }^{2}$, O. Roche ${ }^{2}$

${ }^{1}$ Université Paris 7 Diderot, Paris, France; ${ }^{2}$ Assistance

Publique-Hôpitaux de Paris, Hôpital Necker, Paris, France;

${ }^{3}$ Inserm U 669, Paris, France

\section{Purpose}

Age over 65 years old is a well-known risk factor for iatrogenic hydroxychloroquine retinopathy. Different studies have recently demonstrated that mfERG is the earliest objective test for this follow-up. It can detect abnormalities when they are still reversible. In addition, it had been proposed that visual field recording could be performed for this follow-up. But it is a subjective examination that can be difficult to achieve in elderly patients. The aim of this prospective study was to evaluate the interest and limits of mfERG in patients over 65 years old that receive hydroxychloroquine for a long period. Material and methods

We included in this study all patients over 65 years old that were recorded from 1 January 2007, for a systematic follow-up of hydroxychloroquine therapy. Inclusion criterion required that there was no clinical evidence of iatrogenic retinopathy and that they had far visual acuity over 20/28. A complete ophthalmologic examination as well as a visual field recording was performed for all patients. mfERGs were performed on a Moniteur Ophtalmologique (Métrovision Inc). Technical quality of the mfERG recordings was estimated relative to noise level of each eye.

\section{Results}

Twenty-eight patients (26 females and 2 males) from 65 to 87 years old (mean $73 \pm 6$ years old) were included. HCQ cumulative dose ranged from 292 to $4,500 \mathrm{~g}$ (mean $1,385 \pm 1,037 \mathrm{~g}$ ). None of these patients presented any preclinical retinal toxicity manifestations during the time of the study. mfERG could not be performed on one patient due to important tremor. When the mfERG was recorded on the 27 patients, the mean noise level was $5 \pm 3 \mu \mathrm{V}$ : Technical quality of the mfERG was sufficient for both eyes from 14 patients $(53 \%)$, for only one eye from 7 patients $(25 \%)$ and mediocre for both eyes from $6(22 \%)$ patients.

\section{Conclusion}

Our results confirm that an age over 65 years old is not a limiting factor for mfERG recording or analysis. A recording of sufficient technical quality was obtained for at least one eye in more than $75 \%$ of our patients. mfERG could be included in the systematic examinations proposed in the follow-up of elderly patients treated by hydroxychloroquine for a long period. This is most important since the risk of iatrogenic retinopathy is higher in this population. More precise tests must be used in this class of age. However, if it is impossible to obtain mfERG recordings with a sufficient quality, conventional electrophysiology must be achieved.

\section{OS4-Oral Paper 6}

Using multifocal ERG ring ratios to establish optimal dosage criteria for hydroxychloroquine retinal toxicity

Jonathan S. Lyons, Matthew L. Severns

Georgetown University/Washington Hospital Center Program in Ophthalmology

\section{Purpose}

To develop a metric for predicting Hydroxychloroquine (HCQ) retinal toxicity by determining which dosage criteria best correlate with retinal dysfunction as assessed by mfERGs.

\section{Methods}

We evaluated 252 eyes of 120 patients either currently taking HCQ or who have discontinued taking HCQ. Some of these patients were followed longitudinally. Evaluation included ophthalmologic examination, mfERG testing, automated visual field testing, digital fundus photography, and other tests as clinically indicated. Retinal dysfunction was assessed using mfERG ring ratios [1]. We classified an eye as having a characteristic HCQ deficit if ring 2 or 3 had a ring ratio greater than the 99th percentile of the normal population and the remaining rings were normal. Patients self-reported body weight was also collected.

\section{Results}

Out of 252 eyes, 56 showed a ring ratio abnormality of the types discussed in reference 1; 38 were of the classic "bulls eye" pattern. Retinal dysfunction, as measured by the mfERG, correlates with cumulative dosage. However, cumulative dosage $/ \mathrm{kg}$ of body weight (CDBW) appears to better predict onset of dysfunction. Of those patients who were found to have retinal toxicity, $80 \%$ had a CDBW greater than $19 \mathrm{~g}$ of $\mathrm{HCQ} / \mathrm{kg}$. We also found that the majority of patients who developed toxicity did so after they had ingested between 19 and $30 \mathrm{~g} / \mathrm{kg}$. Of those who returned for evaluation, one patient who developed toxicity by our criteria reverted to normal. The remainder of the affected patients who were re-evaluated showed no further deterioration. 


\section{Conclusion}

Cumulative dose of $\mathrm{HCQ}$ per $\mathrm{kg}$ of reported body weight $(\mathrm{CDBW})$ is an effective metric for predicting retinal abnormalities measured by mfERG. This method provides a relatively sharp demarcation between eyes at lower risk and those at higher risk in our patient population. Moreover, it suggests a dosage range at which the majority of patients appear to be susceptible. Above this range, the percentage of patients who have retinal toxicity appears to decline. It may be that those who are more susceptible have already discontinued the medication. This would explain the decreased percentage of damage in these very long-term HCQ patients as presented in our original paper [1]. It further suggests that patients have different susceptibility factors. After following a smaller number of patients longitudinally, it appears that recovery from the effects of HCQ toxicity can occur when the medication is stopped. Even in those cases where improvement did not occur, there was no further deterioration once the medication was stopped.

\section{Reference}

1. Lyons JS, Severns ML (2007) Detection of early hydroxychloroquine retinal toxicity enhanced by ring ratio analysis of multifocal electroretinography. Am J Ophthalmol 143:801-809

\section{Oral Session 5: Optic nerve and glaucoma}

\author{
Chairs: Mitchell Brigell \& Graham Holder
}

\section{OS5-Oral Paper 1}

\section{Visual electrodiagnosis and visual outcome in optic nerve hypoplasia (ONH)}

\author{
Daphne L. McCulloch ${ }^{1}$, Pamela Garcia-Filion ${ }^{2}$, Cassandra \\ Fink $^{2}$, Caroline A. Chaplin ${ }^{1}$, Mark S. Borchert ${ }^{2}$ \\ ${ }^{1}$ Vision Sciences, Glasgow Caledonian University, \\ Glasgow, Scotland, UK; ${ }^{2}$ Childrens Hospital Los Angeles, \\ and Keck School of Medicine, University of Southern \\ California. Los Angeles, CA
}

\section{Purpose}

In optic nerve hypoplasia $(\mathrm{ONH})$, the congenital dysplasia of the retinal ganglion cells, visual prognosis cannot be determined solely by anatomical examination. Visual electrodiagnostic test in infants and toddlers with $\mathrm{ONH}$ were compared with visual acuity outcome at five years of age.

\section{Methods}

Eighty-six children with $\mathrm{ONH}$ have completed Childrens Hospital Los Angeles long-term prospective study of prenatal and clinical risk factors in $\mathrm{ONH}$. They were recruited before 36 months of age and have now completed initial assessments including ERGs and VEPs to photopic flash and pattern-reversal checks as well as ocular photometry, and biometry with chloral hydrate sedation. Participants also had annual comprehensive assessments. ONH severity was rated as severe, moderate, mild, or fellow eye based on the disk diameter-to-disk macula ratio (DD/DM; respective ratios were $\leq 0.15,0.16-0.30$, $0.31-0.35$, and $>0.35$ ). The final LogMAR visual acuity (VA) was recorded after 5 years of age. To exclude amblyopia as the aetiology of visual impairment $(94 \%$ of the children had strabismus), the better-seeing eye of each child was analysed.

\section{Results}

Participants included 15 children with unilateral and 71 children with bilateral ONH. Based on DD:DM, 76 eyes were classified as severe, 62 as moderate, 11 as mild $\mathrm{ONH}$ and 15 fellow eyes. The flash VEP was a very strong prognostic indicator of visual outcome; large amplitude, clearly reproducible, flash VEPs were associated with good LogMAR VA and those with small, poorly reproduced flash VEPs had a very poor prognosis (Spearman's rank correlation, $P<0.001$ ). For initial PERGs, the amplitude of the N95 component for large checks was predictive of VA at 5 years of age $(P<0.02)$. Photopic flash ERGs were of generally good quality and had no prognostic value. Pattern VEPs were poorly reproduced in most eyes and did not contribute to the assessment of visual prognosis.

\section{Conclusions}

The VEP to flash recorded in infancy or early childhood is useful for establishing the visual prognosis at 5 years of age in children with ONH. In addition, the present data support the hypothesis that the N95 component of the pattern ERG arises primarily from the retinal ganglion cells. N95 is selectively diminished in $\mathrm{ONH}$ and linked to the severity of visual impairment.

\section{Support}

The One Small Voice Foundation, the Carngie Trust for the Universities of Scotland and by NIH General Research Center (GCRG) Grant (M01 RR00043).

\section{OS5-Oral Paper 2}

The optic nerve head component of the porcine multifocal electroretinogram

Rita Buffa ${ }^{1}$, Aphrodite Dracopoulos ${ }^{1,2,3}$, Shelley Boyd ${ }^{1}$

${ }^{1}$ Visual Electrophysiology Unit, St. Michael's Hospital, Toronto, ON; ${ }^{2}$ Department of Respirology, St. Michael's Hospital, Toronto, ON; ${ }^{3}$ Institute of Medial Science, University of Toronto, Toronto, ON

\section{Purpose}

(1) To determine the existence of, and describe, the Optic Nerve Head Component (ONHC) (Bearse and Sutter 1999) of the mfERG of the pig and (2) to determine the influence of inhalational anesthetic agents on this response. The ONHC has not been extensively described, but can be used to measure optic nerve head damage arising from inner retinal elements, including the retinal ganglion cells (RGCs). Because RGCs are damaged in multiple optic neuropathies, and are suggested to be sensitive to anesthetic agents such as isofluorane, we compared two anesthetic protocols on the Ring 2 and Ring 3 response. No study to date has described isofluorane the porcine mfERG or evaluated the effect of anesthetic agents on this response. 


\section{Methods}

A total of 8 six-week-old Yorkshire pigs are included in this study, and divided into two experimental arms. Four pigs received ketamine $(22 \mathrm{mg} / \mathrm{kg} \mathrm{IM})$ with isofluorane (1.5-2.5\%) with $100 \%$ oxygen; investigations are underway with the remaining four using propofol $(12-20 \mathrm{mg} / \mathrm{kg} / \mathrm{h})$ with midazolam $(0.6-1.5 \mathrm{mg} / \mathrm{kg} / \mathrm{h}), \pm$ pancuronium bromide $(0.02-$ $0.06 \mathrm{mg} / \mathrm{kg} / \mathrm{h}$. For all recordings, Burian-Allen contact lens electrodes were used, grounded with a reference needle posterior to the ear. The ONHC stimulus display consisted of 103 equally sized hexagons using the VERIS 6.0 Software from EDI (Electro-Diagnostic Imaging, San Mateo, CA). Two conjunctival sutures were placed to control ocular alignment during visual electrophysiology recording, as needed. Two infrared sources were used to image the optic nerve head.

\section{Results}

The ONHC was readily identified, and conformed to previously published studies (in macaque and human) of Rings 2 and 3, showing increased latencies proportional to the hexagon distance from the optic nerve head. Three peak Amplitudes of the P1 peak were inversely proportional to the isofluorane dose, decreasing by up to $30 \%$ at the maximum $(2.5 \%)$, and recovering with wash-out. Studies with propofol/midazolom are underway.

\section{Conclusions}

This study confirms the presence of the ONHC of the mfERG in the cone-dominant retina of the full-size swine. It demonstrates a relative temporal shift consistent with distance from the optic nerve head, suggesting its dependence on retinal ganglion cell function. Isofluorane, which is well described to reduce amplitudes of the central nervous system (e.g., by electro-encephalography, EEG) consistently reduced the inner retinal response in our model. By contrast, the full-field ERG, which is predominantly derived from the outer retina, was previously demonstrated (Buffa et al. 2004) to be unaffected by this anesthetic agent. In conclusion, these data suggest that the anesthetic protocol is a key variable in the design of potential human studies or clinical trials of inner retinal and optic nerve electrophysiology.

\section{OS5-Oral Paper 3}

\section{Decrease in sweep VEP acuity and contrast sensitivity in ocular hypertensive rabbits-induced by application of carbopol}

K.-M. Zhang, L. Ruiz, A. Vilupuru, B. Feldmann, W. Orilla, T. Lin, Y. Li, C. Spada, M. Escobar, C. Ghosn, J. Burke

Department of Biological Sciences, Allergan, Inc., Irvine, CA

\section{Introduction}

Intracameral carbopol was used to increase intraocular pressure in rabbits to produce a glaucomatous model, as reported by $\mathrm{Xu}$ et al. (2002). Structural and functional changes were monitored over 14 weeks. This study examined the neurosensory and anatomical consequences of high IOP induced by injection of Carbopol.

\section{Methods}

$50 \mu 10.3 \%$ Carbopol 934P was injected into the anterior chamber of the right eye of 28 sedated Dutch-Belted rabbits, using a $33 \mathrm{~g}$ needle. IOP was measured in conscious animals using a pneumatonometer (Reichter Model 30). Retinal nerve fiber layer thickness and optic nerve cup depth were measured using the Topcon 3D OCT in sedated animals (Ketamine/Acepromazine: 15 and $1 \mathrm{mg} / \mathrm{kg}$, respectively). Sweep VEP was recorded in conscious animals using the PowerDiva system with spatial and contrast gratings. Data were represented in mean $\pm \mathrm{sd}$.

\section{Results}

IOP increased from $27.9 \pm 1.4$ to $62.5 \pm 5.0(\mathrm{mmHg})$ at 2 weeks post-injection of carbopol, and gradually resolved back to normal levels by 9 weeks post-injection. IOPs in control eyes remained unchanged. RNFL thicknesses were $20 \pm 5,17 \pm 8$, $13 \pm 8 \mu \mathrm{m}$ in injected eyes and $34 \pm 7,33 \pm 6,33 \pm 5 \mu \mathrm{m}$ in control eyes. Cup depths were 1,183 $\pm 264,1096 \pm 247$, $1,178 \pm 280 \mu \mathrm{m}$ in injected eyes and $833 \pm 172,714 \pm 98$, $755 \pm 101 \mu \mathrm{m}$ in control eyes at 5, 9, and 13 weeks postcarbopol treatment. Sweep VEP acuities were $2.4 \pm 0.6$, $2.3 \pm 0.6,1.9 \pm 0.8$ cycles per degree (cpd) in injected eyes and $2.4 \pm 0.6,2.8 \pm 0.5,2.9 \pm 0.5 \mathrm{cpd}$ in control eyes, while contrast sensitivities were $22.7 \pm 20.8, \quad 22.3 \pm 19.5$, $20.5 \pm 11.8 \%$ ( 1 over contrast threshold in percentage) in injected eyes and $45.7 \pm 29.5,53.9 \pm 65.0,44.3 \pm 22.7 \%$ in control eyes at 6,8 , and 14 weeks post injection.

\section{Conclusion}

Intracameral administration of Carbopol induced dramatic IOP increases, which returned to control levels after about 2 months. However, structural and functional changes continued to progress even after IOP returned to normal levels.

\section{Reference}

1. Xu Y, Chen Z, Song J (2002) A study of experimental carbomer glaucoma and other experimental glaucoma in rabbits. Zhonghua Yan Ke Za Zhi 38:172-175

\section{OS5-Oral Paper 4}

\section{Long-term study of multifocal electroretinographic} changes in experimental glaucoma in macaques

T. Michael Nork, Charlene B. Y. Kim, Gregg A. Heatley, Paul L. Kaufman, Mark J. Lucarelli, Leonard A. Levin, James N. Ver Hoeve

Department of Ophthalmology and Visual Sciences, University of Wisconsin School of Medicine and Public Health, Madison, Wisconsin, USA

\section{Purpose}

To evaluate the effect of chronic experimentally elevated intraocular pressure (IOP) on the mfERG in two species of macaque monkeys.

\section{Methods}

Experimental glaucoma was induced in four rhesus and four cynomolgus monkeys by laser trabecular meshwork destruction (LTD) in one eye. One monkey of each species had undergone prior optic nerve transection (ONT) via a lateral orbitotomy. Durations of elevated IOP varied from 8.5 to 53 months. Each 
animal underwent from 7 to $41 \mathrm{mfERG}$ tests during the period of elevated IOP. mfERGs were recorded under ketamine and pentobarbital anesthesia. A reversing ophthalmoscope was used to align the visual axis of the eyes with a stimulus monitor displaying 103 unstretched hexagonal elements. The VERIS $4.9^{\mathrm{TM}}$ fast $\mathrm{m}$-sequence was employed with a length of $2^{14}$ to 1 at a frame rate of $75 \mathrm{~Hz}$. Two of the non-transected monkeys underwent IOP lowering with a trabeculectomy procedure.

\section{Results}

The ratio of interocular root mean square (RMS) amplitude was relatively constant within epochs of increased intraocular pressure despite large variations in RMS amplitude across test sessions. Repeated measures analysis of variance with maximal reduction of degrees of freedom for correlated measures showed effects of Eye and Eye $\times$ Ring interaction for many of the parameters. Effects of various specific waveform parameters were confirmed by conservative non-parametric Wilcoxon signed ranks tests. Two predominant effects of IOP elevation on the mfERG waveforms were evident. First, there was an increase in amplitude of the early mfERG waveforms (N1 and $\mathrm{P} 1$ ) of the first order kernel (K1). The enhanced K1 RMS amplitude following elevated IOP was more common in the rhesus than in the cynomolgus monkeys. Second, the RMS of the second order kernel, first slice (K2.1), was markedly reduced in all 4 of the cynomolgus monkeys but only variably so in the rhesus monkeys. Both these changes were evident shortly after IOP elevation and were stable with time. IOP effects on the mfERG waveforms in the 2 animals that previously underwent optic nerve transection were similar to those of the nontransected animals. In the 2 animals that underwent IOP lowering by trabeculectomy, the supranormal N1-P1 waveforms of the K1 RMS returned to levels seen at baseline. However, the depressed K2.1 RMS in the cynomolgus monkey did not recover.

\section{Conclusions}

Numerous alterations of mfERG waveforms are seen in response to long-term experimental IOP elevation in nonhuman primates. Some variations in response to IOP elevation appear to be species-specific. The most prominent IOP-related changes are in the early K1 RMS in the rhesus and the K2.1 RMS in cynomolgus monkeys. Owing to the small number of subjects, it is not possible to determine definitively whether this is a true species difference or simply individual animal differences. These results demonstrate that changes in conedriven retinal function during periods of elevated IOP can occur in the absence of retinal ganglion cells and that these changes are largely reversible.

\section{OS5-Oral Paper 5}

\section{Multifocal-OPS of a 2 global flash stimulus in POAG and NTG}

Anja M. Palmowski-Wolfe, Margarita G. Todorova, Selim Orguel, Josel Flammer

University of Basel, Eye Hospital, CH 4031 Basel, Switzerland

\section{Purpose}

To analyse the sensitivity of the mfOPs of the ' 2 global flash' mfERG to detect glaucomatous dysfunction in normal tension
(NTG) and high tension primary open angle glaucoma (POAG) patients.

\section{Methods}

MfERGs were recorded from 20 NTG and 20 POAG patients and compared to those of 20 controls. The mfERG array consisted of 103 hexagons. Each m-sequence step started with focal flash that could be either dark or light (m-sequence: $2^{13}$, $L_{\text {max }}: 200 \mathrm{~cd} / \mathrm{m}^{2}, L_{\min }: 1 \mathrm{~cd} / \mathrm{m}^{2}$ ), followed by two global flashes $\left(L_{\text {max }}: 200 \mathrm{~cd} / \mathrm{m}^{2}\right)$ at an interval of $\sim 26 \mathrm{~ms}$. To extract OPs, data were retrospectively filtered at 100 to $300 \mathrm{~Hz}$. Focal scalar products (SP) were exported for the response to the focal flash, the direct component at $10-40 \mathrm{~ms}$ (DC) and the following two components induced by the effects of the preceding focal flash on the response to the global flashes at 40-70 ms (IC-1) and at 70-100 ms (IC-2) (VERIS 4.8). For each epoch examined, retinal function was considered significantly altered, when more than 5 of the 103 focal areas had a $z$-score of $>+2 /<-2$. In addition, we analysed 5 response averages (central 7.5 degrees and 4 adjoining quadrants). In order to allow small, local areas of dysfunction to show up, responses were normalized by dividing each individual's focal responses by the individual's mean overall response of the corresponding epoch length.

\section{Results}

In all groups, OPs were highest in the central $7.5^{\circ}$. Absolute values of the focal responses or the 5 response averages did not differ significantly between the groups (mixed ANOVA). Focal normalized mfOPs differed significantly between the groups $(3 \times 2$ contingency table). This held true for the DC $(P=$ $0.029)$, the IC-1 $(P=0.001)$ and the IC-2 $(P=0.019)$.

\section{Conclusions}

Focal impairment of mfOPs can be detected in both NTG and HTG. These changes contribute to the glaucomatous retinal dysfunction that has been observed in the 2 global flash multifocal OP.

Support

A grant from Pfizer (PW, TM)

\section{OS5-Oral Paper 6}

\section{Pattern ERG in glaucoma suspects-new findings from a longitudinal study}

Michael Bach, Thomas Jehle

Sektion Funktionelle Sehforschung,

Universitäts-Augenklinik Freiburg, Germany

\section{Purpose}

Early detection of glaucoma remains a challenging problem. We have followed eyes with ocular hypertension (OHT) for 7 years and analyzed the change over time in PERG parameters. Methods

63 OHT eyes (IOP > $21 \mathrm{mmHg}$ ) were followed for 6.6 years on average. We obtained visual acuity, visual fields, morphology (cup-disc ratio and HRT), and the PERG at two check sizes $\left(0.8 \% 16^{\circ}\right)$ to checkerboard reversal at $15 \mathrm{rps}$. Conversion from OHT to manifest glaucoma was defined by one demanding criterion just based on visual fields ("perimetric glaucoma"), and another "pre-perimetric glaucoma" criterion taking also fellow eye status, optic disc morphology, and the HRT into 
account. The patient population was inhomogeneous: a number received diverse medication, some underwent surgery.

\section{Results}

Over the time course analyzed, 20 eyes converted to preperimetric glaucoma, and only 4 eyes to perimetric glaucoma. Receiver operating curves (ROC) showed that the "PERG ratio" $\left(0.8^{\circ} / 16^{\circ}\right.$ amplitudes $)$ predicted conversion better than raw PERG amplitude for pre-perimetric and perimetric glaucoma. The three groups showed the following slopes over the observation period (in the sequence OHT, pre-perimetric and perimetric glaucoma, mean slope $\pm \mathrm{SD}$ ):

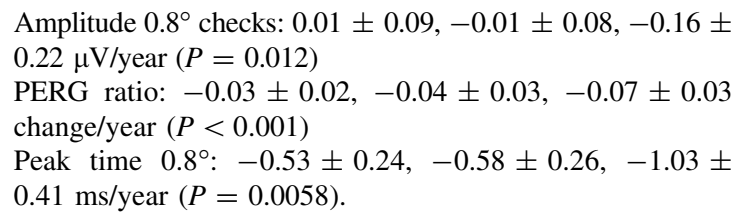

An unexpected finding was the significant decline in peak time over the analysis period. Still, the PERG ratio was more successful in predicting glaucoma than the peak time.

\section{Conclusions}

The results confirm previous findings that the PERG is highly suitable to detect early glaucoma damage. Time series analysis shows a systematic decline of PERG amplitude and PERG ratio in nearly all patients who developed glaucoma, and also in some of those who did not develop manifest glaucoma in the present time interval. The PERG ratio is less useful after manifestation of glaucoma because then amplitude to $16^{\circ}$ checks also start to decrease. The reduction of peak time seems to be of less diagnostic usefulness, but is in keeping with animal data showing a reduction of P50 peak time when spiking activity is inhibited.

Support

The German Research Council (Ba 17-1)

\section{Oral Session 6: Frontiers in electrophysiological testing}

Chairs: Carol Westall \& David Keating

\section{OS6-Oral Paper 1}

The single and multifocal $\mathrm{m}$-sequence technique and their uses in visual electrophysiology

Erich E. Sutter

University of California, San Francisco

\section{Purpose}

To provide an easy to understand explanation of the $\mathrm{m}$-sequence technique, the interpretation of the resulting data and its uses in visual electrophysiology.

\section{Methods}

The multifocal m-sequence technique with its foreign terminology alluding to intimidating mathematical machinery is still puzzling to many of its users. Of particular concern is usually its relation to the familiar conventional approaches that use responses to periodically presented stimuli processed with time-locked averaging. This presentation will show that the connection between the two is quite simple and easy to understand. It will show how an easy translation between the kernels of the m-sequence technique and the results derived with conventional averaging can provide the key to the puzzle. A single short run with $\mathrm{m}$-sequence stimulation can accurately predict the results from not just one but several conventional tests. For instance, the recorded time series may be converted into an isolated flash response, the results from a double or multi-flash experiment or the response to periodic flicker. Such conversions are equally possible when the m-sequence method is used with a Ganzfeld stimulator, a single focus or a multifocus stimulator. Modifications of the technique will be discussed that provide an insight into the complex fast dynamics of the retina and the visual pathway.

Summary and conclusion

Some of this material has been either published before $[1,2]$ or presented at ARVO conferences. In many cases the audience found it difficult to understand. This presentation, illustrated with numerous examples and practical applications, will explain it in the easiest and most comprehensive manner.

\section{References}

1. Sutter E (2000) The interpretation of multifocal binary kernels. Doc Ophthalmol 100(2-3):49-75

2. Sutter EE (2001) Imaging visual function with the multifocal m-sequence technique. Vision Res 41(10-11):1241-1255

\section{OS6-Oral Paper 2}

\section{Mydriasis does not impair multifocal VEP recordings}

Thomas Meigen, Anneliese Friedrich

Department of Ophthalmology, University of Würzburg, Germany

\section{Purpose}

Mydriasis is associated with an increased illuminance level and a reduced retinal image quality. Thus the ISCEV standards and guidelines recommend mydriasis to boost full-field and mfERG responses to flash stimuli, but advise nonmydriatic conditions for PERG and VEP recordings of patterned stimuli. We asked whether both retinal and cortical visual function can be tested under identical mydriatic conditions within the same session by studying the effect of mydriasis on mfERG and mfVEP responses. Methods

11 visually normal subjects participated in the experiment. Multifocal flash and pattern stimuli were presented in a dartboard arrangement of 60 stimulus fields with minimum and maximum luminance values of 0 and $345 \mathrm{~cd} / \mathrm{m}^{2}$. Monocular mfERGs and mfVEPs were recorded simultaneously with one DTL electrode and 4 occipital electrodes that were placed $4 \mathrm{~cm}$ to the left, right, above, and below the inion. The best signal-tonoise ratio was found for a Laplacian-like combination of 3 occipital VEP channels which was used for further analysis. For each recording we calculated a multifocal power function (MPF) by summing the squared signals from all 60 stimulus fields. The sudden MPF rise from baseline indicated the onset of retinal or cortical activity and was used to define latency. Amplitude was quantified by the mean MPF value in a time 
window between 0 and $100 \mathrm{~ms}$ (mfERG) or between 50 and $200 \mathrm{~ms}$ (mfVEP). During the session the pupil was dilated with tropicamide $(0.5 \%)$. Optimal refractive corrections were applied in both mydriatic and non-mydriatic conditions and compensated for the reduced accommodation during mydriasis. We used the Wilcoxon signed rank test to evaluate the statistical significance of differences between mydriatic and non-mydriatic recordings.

\section{Results}

(1) For flash mfERG recordings, mydriasis induced a significant increase in amplitude by a median factor of 3.7 (individual values between 1.7 and $24.5, P=0.003$ ), but no significant change in latency $(P=0.59)$. (2) For pattern mfVEP recordings, mydriasis induced a significant shortening of the latency by a median difference of $3.7 \mathrm{~ms}$ (individual values between -1.2 and $7.0 \mathrm{~ms}, P=0.004)$, but no significant change in amplitude $(P=0.48)$.

\section{Conclusions}

Mydriasis does not lead to significant amplitude changes in mfVEP recordings if appropriate refractive corrections are applied. The decrease in mfVEP latency may reflect a faster retinal processing of pattern stimuli under mydriatic conditions due to the increased illuminance level. The data of our study suggest that mydriatic conditions allow to evoke flash mfERG responses optimally without impairing pattern mfVEP responses which may help to study visual pathway function within the same session.

\section{OS6-Oral Paper 3}

\section{Accommodation limits induced optical defocus in defocus experiments}

Herbert Jägle, Thomas Brauns

Centre of Ophthalmology, University Tübingen, Tübingen, Germany

\section{Purpose}

To study the changes in accommodation during experiments (multifocal VEP) utilizing induced optical defocus.

\section{Method}

Accommodation and pupil size were recorded while running a typical multifocal VEP measurement in 4 healthy subjects using the PowerRefractor (provided by F. Schaeffel, University Tübingen, Germany). A mirror beside the stimulus display was used to image the pupil and a correction for of-axis measurement was applied to the refraction value. We measured refraction and pupil size in emmetropy (correction for the stimulus presentation distance of $0.33 \mathrm{~m}$ was applied) and with additional dioptrical defocus of $+3 \mathrm{D}$. For comparison, we induced cycloplegia with tropicamide and repeated the measurement in emmetropy.

\section{Results}

In emmetropy, pupil size varied between below 3 and ca. $5.5 \mathrm{~mm}$, and the refraction showed fluctuations of up to 3 diopters. With additional defocus we found less variation in pupil size, but higher fluctuations in refraction (8 diopters between minimum and maximum). In cycloplegia, we still find significant fluctuations in refraction.

\section{Conclusions}

The amount of defocus induced with lenses of positive power is limited in non-mydriatic (natural pupil) experiments by accommodation and actual pupil size and may show marked variation during measurement. When studying the effect of defocus on visual function using psychophysical, electrophysiological or functional MRI methods, the use of a mydriatic drug and, if required, an artificial pupil is strongly recommended.

Support

Grant Sh23/6-2 of the German Research Council.

\section{OS6-Oral Paper 4}

\section{Intricacies of measuring the mfERG's luminance uniformity in direct and Maxwellian viewing}

Fabrice Moret, Wolf A. Lagrèze, Michael Bach

Universitäts-Augenklinik, Freiburg, Germany

\section{Purpose}

The amplitude of mfERG recordings shows a decrease with eccentricity, primarily owing to the decreasing density of photoreceptors and neural cells. However, another factor also contributes to this eccentric decrease: the non-uniformity of the stimuli luminance. Different optical setups are used to stimulate in mfERG: CRTs, SLOs, and Maxwellian view systems, each with specific luminance characteristics. Hence a proper calibration method is required. The spotmeter used for measuring CRTs is not always suitable for SLOs and Maxwellian view systems because of the limiting optical geometry of the stimulators. We here propose a new universal non-uniformity measurement method.

\section{Methods}

Spatial luminance data are obtained from recording a mfERG from a $\mathrm{V} \lambda$ corrected photodiode. We first reviewed the geometrical optics at play in a uniformity measurement and derived a correction factor for the resulting intensity maps. The correction factor accounts for the Lambert's cosine law (cos factor), for the difference of distance hexagon-photodiode ( $\cos ^{2}$ factor), and for the virtual tilt of the photodiode regarding the axis hexagonphotodiode (cos factor), all when considering geometrical differences between measuring a central and an eccentric hexagon. We tested its validity by comparing such intensity maps with ISCEV-compliant spotmeter measurements on a CRT.

\section{Results}

The photodiode signal can be treated like a normal ERG if an appropriate low-pass filter is applied; a high-pass cutoff frequency of $120 \mathrm{~Hz}$ was suitable. The corrected data retrieved by the mfERG record of the photodiode response differs by approximately $5 \%$ when compared with the spotmeter data. This difference was $1 / 3$ rd of the ISCEV-recommended $15 \%$ maximal non-uniformity.

\section{Conclusions}

The method introduced here allows measuring the luminance uniformity of mfERG stimuli in CRTs, SLOs, and Maxwellian view systems. It relies on the combined use of a photodiode and an m-sequence. Comparison to the spotmeter measurement on CRT validated the geometrical model. The method is simple and inexpensive ( $<300$ EUR), and can be operated by medical staff. 


\section{OS6-Oral Paper 5}

\section{Light adaptation and dark adaptation of the human visual system}

Rasa Ruseckaite, Allison M. Cameron, Liang Miao, Trevor D. Lamb

Division of Neuroscience, John Curtin School of Medical Research, and ARC Centre of Excellence in Vision Science, Australian National University, Canberra ACT 0200, Australia

\section{Purpose}

(1) To study psychophysical light adaptation and dark adaptation in individual subjects whose ERG b-wave responses had also been measured. (2) To compare the post-bleach recovery of 'equivalent background intensity' (extracted using a 'Crawford transformation') from the psychophysical and ERG b-wave measurements.

\section{Methods}

We developed a Matlab GUI program to control a commercial desktop ganzfeld and measure thresholds, using an automated staircase procedure. Blue test flashes $\left(\lambda_{\max }\right.$ $=455 \mathrm{~nm}$ ) delivered within the stimulator, were presented either full-field or through a mask $\left(5^{\circ}\right.$ dia., centred $12^{\circ}$ in the nasal field). Psychophysical light adaptation was measured using backgrounds of the same colour and geometry, at a range of luminances from 105 to $104 \mathrm{Td}$. Psychophysical dark adaptation was measured after ganzfeld bleaches calculated to range from $\sim 1$ to $\sim 90 \%$. The scotopic b-wave of the ERG was recorded in response to ganzfeld stimulation with $\operatorname{dim}$ blue flashes $\left(\lambda_{\max }=470 \mathrm{~nm}\right)$, either under fully dark-adapted conditions, during adaptation to steady backgrounds, or during dark adaptation recovery following bleaching exposures comparable to those used in the psychophysical experiments (Cameron et al. (2006) J Physiol 575:507-526). Bleaching levels were estimated using the MLP rate-limited model of pigment regeneration (Mahroo and Lamb (2004) J Physiol 554:417-437) with parameters $Q_{\mathrm{e}}=106.8 \mathrm{Td} \mathrm{s}, \mathrm{v}=0.085 \mathrm{~min}^{-1}$ and $K_{\mathrm{m}}=0.2$.

\section{Results}

Under scotopic conditions, a Weber-law exponent $\mathrm{n}$ for psychophysical light adaptation varied between 0.8 and 1.1 in three subjects tested, both for the small field and for ganzfeld illumination. In psychophysical dark adaptation experiments, the slope of the ' $\mathrm{S} 2$ ' component of recovery was $\sim 0.24$ decades min 1 , but after conversion to equivalent background intensity this slope increased marginally. The time taken to recover to a criterion level (of threshold, or of equivalent background) increased approximately linearly with the size of the bleach for each subject. Furthermore, the form of the relationship was similar to that obtained in the ERG b-wave experiments.

\section{Conclusions}

The post-bleach recovery of equivalent background intensity, measured psycho-physically or using the ERG b-wave sensitivity, is similar for the individual subjects studied.

\section{OS6-Oral Paper 6}

\section{Precisely specifying the bandwidth of electrodiagnostic recordings}

\author{
A. C. Fisher, S. P. Lake, R. P. Hagan, M. C. Brown \\ Clinical Engineering Dept. and Clinical Eye Research \\ Centre, Royal Liverpool University Hospital, Liverpool, UK
}

\section{Introduction}

Recent discussions in the ophthalmic electrophysiology community (CEVNET) have brought into question the appropriateness of the most commonly used bandwidths for recording and reporting transient response examinations. Whilst bandwidths are specified in ISCEV methodology recommendations, the scientific rationale for these specifications is somewhat difficult to justify. Very loose definitions of frequency response are used, most often with little attention to order and phase response. Consequently, across a range of laboratories using differing equipment, the identification of 'information' from the 'data' of recordings is variable, and establishing realistic inter-institutional norms and standards is unrealistic. This is clearly a legacy of analogue signal processing. However, modern and available digital computing can provide explicitly specified analyses which are easily implemented and completely circumvent these limitations. The requirement is to introduce the theory of these techniques to the critical clinical community and to equipment manufacturers, and to provide practically realisable tools so that clinical best practice can advance.

\section{Methods}

The mfERG and the PERG are taken as examples. The effects of 4 bandwidth-limiting models are illustrated graphically:

I. finite impulse response filter (FIR, constant phase);

II. infinite impulse response filter (IIR, Butterworth-like phase);

III. Fourier Series decomposition;

IV. discrete wavelet decomposition.

These models will be presented in 'real-time' as web-based Internet-accessible procedures, freely available as 'open source' and fully functional either from a simple web browser using MatSOAP technology or a local program call to a remote server implementation (RPC).

\section{Results}

The effects of differing bandwidth-limiting regimes of ad hoc user-chosen specifications applied to exemplar clinical PERGs and mfERGs with a range of signal-to-noise ratios will be demonstrated graphically without recourse to unfamiliar mathematics. The relative sensitivity and robustness of cardinal point identification ('cursoring positions') in noise will be illustrated. A working 'click-and-go' (sic) RPC example of FIR filtering (Method i) called from the Espion Instrument (Diagnosys LLC) will be demonstrated for a series of clinical PERGs.

\section{Conclusions}

Digital processing allows the frequency response of electrodiagnostic records to be explicitly and precisely specified. These 
techniques are easily incorporated into existing analysis software either locally or over the Internet.

\section{Poster Session B: retinal diseases-ERG methods}

\author{
Chairs: Monique Leys \& Neal Peachey
}

\section{Poster B1}

\section{Study of the cone dysfunction in eyes with macular edema}

Weiju Wu ${ }^{1}$, Feng Wen ${ }^{2}$, Shizhou Huang ${ }^{2}$, Guangwei Luo ${ }^{2}$, Dezheng $\mathrm{Wu}^{2}$

${ }^{1}$ School of Biological and Biomedical Sciences,

Durham University, Durham, DH1 3LE, UK;

${ }^{2}$ Zhongshan Ophthalmic Center, Sun Yat-Sen University, Guangzhou, 510060, China

\section{Purpose}

To study the impaired cone function by mfERG and FM 100hue test and the S-cone function by S-cone ERG in the eyes with macular edema caused by diabetic retinopathy (DR) and Behcet disease and detect the differences between the cystoid macular edema (CME) and diffuse macular edema.

\section{Methods}

Macular edema was divided into CME and diffuse macular edema according to fundus fluorescein angiography (FFA). 19 patients ( 35 eyes) with Behcet disease and 18 patients ( 30 eyes) with DR had CME. 20 patients (32 eyes) with Behcet disease and 18 patients (32 eyes) with DR had diffuse macular edema. 42 normals (49 eyes) were included as the control subjects. mfERG, FM 100-hue, and S-cone ERG were performed on all patients. The latter was recorded by blue flashes $(\lambda=465 \mathrm{~nm}$, stimulus intensities of $0.05 \mathrm{~cd} \mathrm{~s} / \mathrm{m}^{2}$ ) and orange background light $\left(\lambda=594 \mathrm{~nm}\right.$, stimulus intensities of $\left.0.05 \mathrm{~cd} \mathrm{~s} / \mathrm{m}^{2}\right)$.

Results

There was no statistically significant difference between the $\mathrm{DR}$ and Behcet disease in the two types of macular edema in the comparison of each results of mfERG, FM 100-hue, and S-cone ERG. Then the patients with two kinds of diseases in each type of macular edema were put together and analyzed as a whole group. The latencies of $\mathrm{N} 1$ and $\mathrm{P} 1$ waves were remarkably prolonged $(P<0.05)$ and the average response densities of $\mathrm{N} 1$ and $\mathrm{P} 1$ waves were seriously decreased $(P<0.05)$ in the two types of macular edema, compared with the normal subjects. There was no statistically significant difference between the diffuse macular edema and CME in the latencies of $\mathrm{N} 1$ and P1 waves $(P>0.05)$. However, there were statistically significant differences in the average response densities of $\mathrm{N} 1$ wave at 5 and 6 rings $(P<0.05)$ and $\mathrm{P} 1$ wave at 4,5 , and 6 rings $(P<0.05)$. There were 69 patients $(121$ eyes) whose error scores were beyond the normal score range in the FM100-hue test. The means and standard deviation of error scores were $205.17 \pm 142.12$ and $232.38 \pm 150.64$ between the CME and diffuse macular edema. However, there was no statistically significant difference between them $(P<0.05)$. Most of the patients exhibited a significant tritan axis according to the axis analyzation. The latency of $b$ wave was remarkably prolonged $(P<0.05)$ and the amplitude was seriously decreased $(P<0.05)$ in the two kinds of macular edema in the S-cone ERG, compared with the normal control subjects. The amplitude of CME is much smaller than that of diffuse macular edema $(P<0.05)$. But there was no statistically significant difference in the latency between them.

\section{Conclusions}

Both the types of macular edema can cause serious cone dysfunction. mfERG can reflect the dysfunction. S-cone ERG can reflect the dysfunction of S-cone system.

\section{Poster B2}

Multifocal electroretinographic and electroretinographic change of affected and fellow eye with central retinal vein occlusion (CRVO)

Manami Kuze, Hisashi Matsubara, Yukitaka Uji

Mie University School of Medicine, Mei, Japan

\section{Purpose}

To determine the change of mfERG recorded in patients with central retinal vein occlusion (CRVO) and to compare the responses of the affected and fellow eyes. In addition, mfERG responses were compared by using full-field ERG.

\section{Methods}

mfERG and ERG responses were recorded from both eyes of 15 patients with CRVO. The mfERG responses, obtained using a custom-built system, were grouped into six rings. The P1 amplitudes and implicit times were grouped and averaged within each ring. Statistical analysis was used to compare the ERG results from the affected and fellow eyes. The results were also compared with normals.

\section{Results}

In the affected eyes, mfERG P1 amplitude decreased (86.3 \pm $7.4 \%)$ and implicit times prolonged $(118.5 \pm 4.0 \%)$ on all the ring area compared to the fellow eyes. In fellow eyes, P1 amplitude decreased only in central ring compared to the normal area, however not in the other ring area. P1 implicit times were prolonged in all rings (average: $113.2 \pm 4.0 \%$ ) and reached to statistical significance $(P<0.005)$. The ERG responses obtained from eyes with CRVO were significantly different $(P<0.01)$ from those derived from the fellow eye and the responses obtained from fellow eye were also different from those of normal eye.

\section{Conclusions}

On CRVO patients, ERG and mfERG revealed abnormal retinal function even in the fellow eyes which showed no specific change as affected eyes. ERG and mfERG could be a sensitive indicator of the underlying disease affecting the retina in fellow eyes with CRVO.

\section{Poster B3}

\section{Foveal thinning in cases with occult macular dystrophy}

Azza Shehab ${ }^{1}$, Mai Sharawy ${ }^{2}$

${ }^{1}$ Minya University; ${ }^{2}$ Cairo University

\section{Purpose}

To determine the correlation between electrophysiology, perimetry, and optical coherence tomography in cases with unexplained bilateral defective central vision. 


\section{Patients and methods}

7 referred patients with unexplained reduced central vision (aided VA: $0.5 \pm 0.2$ ) were included in the study. All patients underwent complete ophthalmic examination, FA, electrophysiology, perimetry, and OCT. 5 patients had mfERG. Color vision test was performed using 100-hue test or Ishihara plates. Results

Mean age of the patients was $14.7 \pm 6.18$. No gender preferences with 3 males and 4 females equally affected. 2 families had 2 affected members. All patients had bilateral reduced visual acuity, normal fundus examination, normal fluorescein angiogram, normal ERG, PVEP, and EOG. All patients had normal optic disc with normal C/D ratio and normal IOP. All patients had PERG, central field, and OCT, all abnormal. All patients who had mfERG had abnormal results. Color vision was average to good with no axis.

\section{Conclusion}

Cases with unexplained defective central vision always represent a diagnostic dilemma in the ophthalmology practice. With the development of wide spectrum of the investigative tools, it became even difficult for the ophthalmologist to pick up the appropriate tools among imaging and functional investigative techniques. The correlation between electrophysiology, perimetry, and OCT is crucial in cases of defective vision due to macular dysfunction.

\section{Poster B4}

Local and multifocal electroretinography and macula imaging in Stargardt's disease/fundus flavimaculatus

I. Zolnikova, E. V. Rogatina, I. Z. Karlova

Helmholtz Research Institute of Eye Diseases, Moscow, Russia

\section{Purpose}

To assess retinal bioelectrical activity in Stargardt's disease/ fundus flavimaculatus and compare it with OCT data.

\section{Material and methods}

34 patients (68 eyes), aged 7-40 years, with Stargardt's disease/fundus flavimaculatus (FFM) were examined. Examination included best-corrected visual acuity (BCVA) estimation, fundus photographs, and molecular genetics. Electroretinography (ERG) according to the ISCEV standards and chromatic macular ERG (m-ERG) was recorded with MBN electroretinographic system (Russia), the mfERG-with RETI scan system (Roland Consult, Germany). Optical coherence tomography (OCT) was performed on Stratus OCT-3 (Carl Zeiss, USA). The patients were divided into three groups based on electroretinographic findings (Lois et al. 2001). Group 1 was characterized with subnormal $\mathrm{m}$-ERGs to red stimulus, and normal $30-\mathrm{Hz}$ flicker and full-field cone-rod responses and included 7 patients (14 eyes), mean age $20.6 \mathrm{yrs}$, BCVA 0.36 (0.1-0.8); Group 2, with the reduced or nonrecordable $\mathrm{m}$ ERGs, reduced $30 \mathrm{~Hz}$ flicker and photopic ERGs, and the normal amplitude of cone-rod full-field ERGs in 7 patients (14 eyes), mean age 15.2 yrs, BCVA 0.24 (0.05-0.8); Group 3, with severely reduced or nonrecordable $30 \mathrm{~Hz}$ flicker and photopic ERGs and subnormal amplitudes of cone-rod fullfield ERGs and scotopic ERGs included 20 patients (40 eyes), mean age 16.8 yrs, BCVA 0.27 (0.02-0.8). Clinical findings included bilateral changes in fovea ranging from loss of foveal reflex to macular atrophy and yellow-white checks located in parafovea or scattered throughout the posterior pole and midperiphery.

\section{Results}

In patients with Stargardt's disease/FFM, OCT data revealed markedly reduced foveal thickness and decreased total macular volume $(P<0.05)$. A strong direct correlation between the amplitude of $\mathrm{b}$-wave of $\mathrm{m}$-ERG and macular volume ( $r=0.89, P<0.001)$ and between the mean $\mathrm{P} 1$ response density from central 3 rings and macular volume $(r=0.94$, $P<0.001)$ indicates that the reduction of macular responses is associated with tissue loss in macula (possibly with atrophy of the neurosensory retina). In patients from group 3 with severe rod dysfunction, paramacular thickness was also reduced, indicating the possible association between rod system function and retinal thickness. There is a strong direct correlation between $\mathrm{b}$-wave $\mathrm{m}$-ERG to red stimulus and mean $\mathrm{P} 1$ response density from central 3 rings of mfERG $(r=0.69, P=0.002)$.

\section{Conclusion}

Reduction of central bioelectric responses in Stargardt's disease/fundus flavimaculatus is associated with reduced macular thickness. M-ERG and mfERG from central rings demonstrate high correspondence.

\section{Poster B5}

Changes in the myopic retina using multifocal electroretinograms, optical coherence tomography and peripheral resolution acuity

Clive J. Wolsley ${ }^{1,2}$, Kathryn J. Saunders ${ }^{1}$, Giuliana Silvestri ${ }^{3}$, Roger S. Anderson ${ }^{1}$

${ }^{1}$ Vision Science Research Group, University of Ulster, Coleraine, UK; ${ }^{2}$ Northern Ireland Medical Physics Agency, Royal Victoria Hospital, Belfast, UK; ${ }^{3}$ Department of Ophthalmology, Centre for Vision Science, Queen's University, Belfast, UK

\section{Purpose}

To investigate relationships between retinal structural and functional change in human myopia.

Methods

Optical coherence tomography (OCT), peripheral resolution acuity and multifocal electroretinography (mfERG) were performed on 56 subjects with a range of refractive errors (+0.50 to -15.00 diopters) in order to generate comparative retinal structure-function data at specific retinal locations within the same eyes. Changes to the retinal laminar structure (retinal thickness) in myopia were compared to changes in retinal function.

\section{Results}

Total retinal thickness was reduced in moderate and high myopia and this correlated with reduced spatial resolution and delayed mfERG timing in the peripheral retina. The reduction in OCT retinal thickness was dominated by a thinning of the mid-inner retina. Foveal topography was altered in high myopia although in general photoreceptor retinal thickness was preserved. 


\section{Conclusion}

Given that peripheral resolution acuity reflects ganglion cell density $(1,2)$ and that bipolar cells contribute to the mfERG response (3), these findings suggest the structure and function of the post-receptor retina are susceptible to disruption in moderately and highly myopic eyes.

\section{References}

1. Williams DR (1985) Aliasing in human foveal vision. Vision Res 25:195-205

2. Thibos LN, Cheney FE, Walsh DJ (1987a) Retinal limits to the detection and resolution of gratings. J Opt Soc Am Opt Image Sci 4:1524-1529

3. Hood DC, Frishman LJ, Saszik S, Viswanathan S (2002) Retinal origins of the primate multifocal ERG: Implications for the human response. Invest Ophthalmol Visual Sci 43:1673-1685

\section{Support}

N.I. Research and Development Office.

\section{Poster B6}

\section{Spontaneous recovery of the photoreceptor inner/outer segment line defect in eyes with multiple evanescent white dot syndrome}

Atsuhiro Tanikawa, Eri Nakayasu, Ryo Asano, Masayuki Horiguchi

Department of Ophthalmology, Fujita Health University, Toyoake, Japan

\section{Purpose}

To report two cases of multiple evanescent white dot syndrome (MEWDS) in which spectral domain optical coherence tomography (SD-OCT) demonstrated the improvements of the photoreceptor inner/outer segment (IS/OS) line defect.

\section{Methods}

The examination included best-corrected visual acuity (BCVA), slit-lamp and fundus examination, Goldmann or micro-perimetry, fluorescein angiography, SD-OCT, and fullfield or multifocal ERGs.

CASE 1: A 20-year-old male had a visual disturbance of acute onset in his right eye. His BCVA was 0.1 (OD) and 1.2 (OS). Microperimetry showed central scotoma in his right eye. There were multiple grayish-white lesions around the post pole in the right eye, and they disappeared in several days. Dark-adapted full-field ERG revealed negative waveform only in the right eye. SD-OCT showed loss of the IS/OS line corresponding to the central scotoma. The defected IS/OS line recovered gradually, as his BCVA and central scotoma improved.

CASE 2: A 29-year-old male had an acute blurred vision in his right eye. His BCVA was 0.1 (OD) and 1.0 (OS). Goldmann perimetry showed ring scotoma and reduced central sensitivity. There were multiple grayish-white lesions around the post pole in the right eye, and they disappeared in a week. Multifocal ERG of the right eye revealed decreased amplitudes compared to the normal left eye. OCT showed the irregularity or loss of the IS/OS line in his right eye. It recovered gradually, as his visual disturbance improved.

\section{Conclusions}

SD-OCT may demonstrate the photoreceptor improvements according to the recovery of visual disturbance in eyes with MEWDS, as well as the photoreceptor disturbances in the acute phase.

\section{Poster B7}

\section{Bilateral choroidal neovascular membrane as a presenting feature of best's disease}

Rohit Shetty ${ }^{1}$, Ajoy Vincent ${ }^{1}$, Rajani R Battu ${ }^{2}$, Bhujang Shetty ${ }^{1}$

${ }^{1}$ Department of electrophysiology, Narayana Nethralaya, Super Specialty Eye Hospital and Post Graduate Institute, Bangalore, India; ${ }^{2}$ Department of Vitreoretina, Narayana Nethralaya, Narayana Health City Campus, Bommasandra, Bangalore, India

\section{Purpose}

To report the role of visual electrophysiologist in the diagnosis of Best's disease presenting as bilateral choroidal neovascular membrane (CNVM).

\section{Methods}

A 14-year-old male who presented with complaints of diminution of vision in both eyes underwent detailed ophthalmological evaluation including Best-Corrected Visual Acuity (BCVA), color vision, fundus evaluation and photography, Ocular Coherence Tomography (OCT), Fundus Fluorescein Angiography (FFA), Indocyanine Green Angiography (ICG), full-field ERG and EOG (Metrovision, France). The ERG and EOG settings satisfied ISCEV guidelines.

\section{Results}

The BCVA was $6 / 18$ in both the eyes. Color vision was normal in both the eyes. Fundus evaluation of the right eye showed elevated pinkish lesion at the macula with retinal edema, surrounding retinal bleed, and serous retinal detachment. Fundus evaluation of the left eye showed atrophic scar at the macula with sub-retinal bleed. FFA of the right eye showed areas of leakage at the macula. These areas of leakage increased in size and intensity with time. FFA of the left eye showed area of leakage and areas of blocked fluorescence at the macula. The rest of the fundus was normal. The OCT showed central retinal edema and subretinal fluid in the right eye. The OCT of the left eye showed CNVM complex. ERG was within normal parameters in both the eyes for all stimulus parameters. The EOG showed markedly subnormal Arden's ratio of $113 \%$ and $115 \%$ in the right and left eye, respectively.

\section{Conclusion}

A diagnosis of vitelliruptive stage of Best's disease with CNVM was made in the right eye and a diagnosis of atrophic stage of Best's disease with CNVM was made in the left eye. Best's disease is an autosomal dominant dystrophy commonly presenting as a vitelliform lesion at the macula. Bilateral CNVM is a very rare complication of Best's disease. The retinal bleed almost completely masked the vitelliruptive lesion in the right eye. The atrophic scar in the left eye could have been misdiagnosed to be secondary to any other retinal pathology. The EOG helped to clinch the diagnosis in this case. 


\section{Poster B8}

Dark adaptometry following implantation of blue blocker and aspheric intraocular lenses

Rohit Shetty ${ }^{1,2}$, Mathew Kurian ${ }^{2}$, Ajoy Vincent ${ }^{1}$, Bhujang Shetty ${ }^{1}$

${ }^{1}$ Department of electrophysiology, Narayana Nethralaya, Super Specialty Eye Hospital and Post Graduate Institute, Bangalore, India; ${ }^{2}$ Department of Cataract and Refractive surgery, Narayana Nethralaya, Super Specialty Eye Hospital and Post Graduate Institute, Bangalore, India

\section{Purpose}

To assess and compare the final visual outcome in terms of visual acuity and dark adaptometry in patients undergoing phacoemulsification with implantation of blue blocker intraocular lens (IOL) and aspheric IOL.

\section{Methods}

A prospective, interventional study was undertaken after Institute Ethics Committee approval. Those patients admitted for cataract surgery that signed an informed consent were included. Exclusion criteria included monocular patients, abnormal corneal endothelial count or morphology, active or prior uveitis, glaucoma and active retinopathy. Twenty eyes underwent phacoemulsification and implantation of blue blocker IOL implantation and another 20 eyes underwent phacoemulsification and implantation of aspheric IOL. The visual outcomes were measured in terms of visual acuity and dark adaptometry at six weeks post operatively. Bestcorrected distance and near vision acuity were recorded. Dark adaptometry was studied using the MetroVision electro-diagnostic system (Perenchies, France). Dark adaptometry was done on a cathode ray tube using spatial dithering. A testing distance of $30 \mathrm{~cm}$ was used and the duration of the light and dark phases were 5 and $30 \mathrm{~min}$, respectively. A $35 \mathrm{~dB}$ filter goggle was worn to test the dark adaptometry.

\section{Results}

The postoperative mean best-corrected visual acuity in the two groups was comparable. There was a reduction in the mean dark adaptation values reached at $20 \mathrm{~min}$ in the blue blocker IOL group as compared to the aspheric IOL group. There was also a reduction in the maximum dark adaptation value attained in the blue blocker group when compared to the aspheric IOL group.

\section{Conclusion}

The incorporation of chromophobes in the IOL materials to protect the retina from age-related macular degeneration does not have significant effect on best-corrected distance and near vision parameters. Reductions in dark adaptometry is of clinical relevance as it may dampen night vision to a significant extent in patients who have significant amount of outer retinal damage, those who have undergone panretinal photocoagulation or those who have inherited rod dystrophies.

\section{Poster B9}

Syndromic choroideremia-a possible approach to sublocalization of phenes associated with Martin-Probst deafness mental retardation syndrome

C. M. Poloschek ${ }^{1}$, B. Kloeckener-Gruissem ${ }^{2,3}$, L. L. Hansen ${ }^{1}$, M. Bach ${ }^{1}$, W. Berger ${ }^{2}$

${ }^{1}$ Department of Ophthalmology, University of Freiburg, Freiburg, Germany; ${ }^{2}$ Division of Medical Molecular Genetics and Gene Diagnostics, Institute of Medical Genetics, University of Zurich, Schwerzenbach, Switzerland; ${ }^{3}$ Department of Biology, ETH Zurich, Zurich, Switzerland

\section{Purpose}

To identify the mutation leading to syndromic choroideremia (CHM) in two families and to define fundus autofluorescence (FAF) in CHM carriers.

\section{Methods}

We investigated the ophthalmological and clinical phenotype including FAF, neuropediatric, otorhinolaryngologic, cardiologic, and nephrologic examinations of 3 male patients (age 16-21 years) and 3 female carriers (age 11-46 years) from two families with syndromic CHM. Full-field ERGs and mfERGs were performed according to the ISCEV standards and guidelines. Genomic DNA amplification (PCR) of the REP1 gene as well as adjacent loci was used to determine the molecular basis of the phenotype.

\section{Results}

In CHM patients, scotopic ERGs were not detectable (both families); the flicker response did not reveal significant cone responses (family 1) or was reduced to $1 / 4$ of the norm (family 2). MfERGs were reduced to $1 / 5$ of the norm (family 1) and showed a pathologic parafoveolar peak time increase (family 2). In CHM carriers, ERG and mfERG amplitudes and peak times were normal. However, one carrier showed a slightly diminished central mfERG amplitude. Analysis of DNA revealed a large deletion which asymmetrically flanks REP1 in both families, ranging from a minimum size of 6.3 and 8.5 megabases to a maximum size of 9.7 and 14.1 megabases, respectively. In addition to $\mathrm{CHM}$, patients from these families also exhibited mild syndromic features including mental and motor retardation as well as low frequency hearing loss. FAF showed a distinctive pattern of small areas of reduced and increased autofluorescence in all female carriers.

\section{Conclusions}

Both CHM families are the first to be described with large deletions manifesting with a mild syndromic phenotype. Based on the location of the deletions, they may allow sub-localization of the syndromic features to the most proximal region of X-linked distal spinal muscular atrophy and Martin-Probst deafness mental retardation syndrome. The FAF pattern is specific to CHM carriers and thus will help to identify and differentiate between carriers of other X-linked recessive carrier states such as in X-linked Retinitis Pigmentosa. 


\section{Poster B10}

\section{The importance of clinical examination of patients who come for electrofunctional test}

\author{
Alma Patrizia Tormene ${ }^{1}$, Morena Masetto ${ }^{2}$, Chiara Riva ${ }^{1}$ \\ ${ }^{1}$ Department of Neurosciences, Ophthalmology University \\ of Padova, Italy; ${ }^{2}$ Ophthalmology of Abano Terme \\ Hospital, Abano Terme, Italy
}

\section{Purpose}

A patient came for electrofunctional test for suspected optic nerve toxicity. Functional results are analysed in relation to ophthalmological examination.

\section{Methods}

Subject, aged 30, affected by undifferentiated connectivitis. Currently undergoing therapy with : Nifedipine, Venlafaxine, Gaviscon. Paroxetina $60 \mathrm{mg} /$ die recently prescribed for depression. Two months later subject reported a reduction of bilateral visual acuity. Objective exanimation showed: visus 0.6 in $\mathrm{OO}$; ocular pressure RE 23, LE $46 \mathrm{mmHg}$. Retinal marked arterioschlerosis, increased optic disc cup, chorio-retinal atrophy with pigment clumps. Therapy advised: Timololo and Acetazolamide, VF, ERG and VEPs and suspension of Paroxetina. The following day the pressure was RE 12 , LE $20 \mathrm{mmHg}$. Visual field revealed large scotomatous areas. After two months, the patient came for electrofunctional tests (late request for appointment); visual acuity had fallen to finger-counting in RE and to 0.4 in LE. The following tests were done: VEPs (recording electrodes: OZ, O1, $\mathrm{O} 2$ reference FZ) and PERG transient pattern reversal (DTL electrodes) at 3 spatial frequencies $\left(67^{\prime}, 46^{\prime}, 10^{\prime}\right)$, ERG: MCR, RR, CR, Flicker Response, PO (DTL recording electrodes), and EOG using standard ISCEV methods.

\section{Results}

VEPs: P 100 markedly reduced, implicit time normal. PERG: absent in OO. ERG: MCR, RR, CR, Flicker R bilaterally markedly reduced. Rod component severely compromised. The patient was re-examined by doctors from the Electrophysiology Service and showed marked reduction in retinal vessels size in the retina and fine optic disc neovascularization in RE and vessels in irido-corneal angle. The results of these electrofunctional tests could suggest iatrogenic retinal toxicity while the reduction in VEPs and PERG could be secondary to the retinopathy or to glaucoma. Alternatively vessel thinning, associated with reduction in ERG, could make one think of a form of retinal heredodegeneration but the presence of the neovascularization, together with glaucoma, led us to suspect an ischemic event. To check this hypothesis EOG and PO were carried out with the following results: PO: absent in OO. EOG: Arden Ratio normal in OO. The fact that EOG was normal excluded a retinal heredodegeneration and the absence of PO confirmed the suspicion of generalized possible ischemic damage. Suspected ischemia was diagnosed, as toxicity seemed unlikely; thus the Patient was advised to continue with Paroxetina. Further tests on the retinal vasal situation were advised. Fluorangiography and ICG revealed marked chorioretinal ischemia, OCT showed global retinal thinning. Further tests were impossible as the patient died from pneumonia.

\section{Conclusions}

A bilateral chorio-retinal ischemia in a patient affected by undifferentiated connectivitis is an association that has not yet been described in the literature even though a particularly aggressive form of a disease of the microcirculation cannot be excluded. Anamnesis would suggest an iatrogenic pathology but clinical examination, together with the results of the electrofunctional examination, allowed us to identify the cause of loss of visus which was a chronic chorio-retinal ischemia. We have reported this case not only because it is unusual but also because it highlights the importance of careful clinical examination of patients who come for electrofunctional tests.

\section{Poster B11}

\section{Skin electrodes vs corneal electrodes (Burian-Allen) for ISCEV standard full-field ERG-determining a method to obtain repeatable results}

Mary Flynn Roberts, Elizabeth Warren, Angela VanDyke

Illinois Eye Institute, Illinois College of Optometry

\section{Purpose}

In order to compare two different methods of gathering the data with full-field ERGs according to ISCEV protocols, we recorded ERGs using corneal electrodes (Burian-Allen) and electrodes placed on the skin of the lower eyelid. We also experimented with methods of obtaining reliable, repeatable ERGs using skin electrodes.

\section{Methods}

Data were collected from 16 normal volunteers between the ages of 24 and 27. All subjects had clear media, the absence of high myopia, and no significant ocular disease. One eye of each subject was dilated using $2.5 \%$ phenylephrine and $1 \%$ tropicamide. Following $30 \mathrm{~min}$ of dark-adaptation an ERG was recorded using a Burian-Allen unipolar electrode and standard ISCEV protocol. Following this test the subject was again dark-adapted for $30 \mathrm{~min}$, and the ERG was measured using a skin electrode on the same eye. The skin electrode was placed on the center of the lower lid. In order to obtain reliable, repeatable readings, we determined that if the subject was asked to tip their forehead backward a little and look slightly down at the stimulus we could obtain repeatable results. In this position, the skin electrode was in closer contact to the cornea, thereby increasing the amplitude of the reading recorded and eliciting a stronger response. To evaluate the results for each patient, we compared the a-wave amplitude, b-wave amplitude, a-wave implicit time, and b-wave implicit time between the corneal and skin electrodes. Repeatable data were acquired from all 16 patients using both the Burian-Allen electrode and the skin electrode.

\section{Results}

The results from the skin electrode ERGs showed longer implicit times as compared to the results from the corneal electrode for the majority of the readings. The median difference between the amplitude values of the two ERG results with the electrodes (corneal $>$ skin) fell between $21 \%$ and $33 \%$. Thus the amplitudes obtained with a skin electrode were approximately $1 / 4$ to $1 / 3$ less than the amplitudes obtained with a corneal electrode.

\section{Conclusion}

A method for obtaining reliable, repeatable ERGs with skin electrodes and standard ISCEV protocols was determined. The 
results of this study will be beneficial for future use of a skin electrode when performing full-field ERGs in our service.

\section{Poster B12}

\section{Electrical impedance measurement on the sclera/cornea}

Malcolm Brown, Laura Milner, Anthony Fisher, Richard Hagan

Clinical Engineering Department and Clinical Eye Research Centre, Royal Liverpool University Hospital, Liverpool, UK

\section{Purpose}

To investigate the methods and currents used to measure electrode impedance especially in relation to scleral/corneal electrodes. This work was undertaken to assist in the debate regarding the risks of measuring electrode impedances at the eye.

\section{Methods}

A number of electrode impedance meters and electrophysiology recording systems in common use in the UK were investigated to establish the methods by which electrode impedance is measured and in particular what currents and waveforms are used.

\section{Results}

In general, the frequency ranges were $10-100 \mathrm{~Hz}$ and currents passed through the electrodes varied from $<1$ to $50 \mu \mathrm{A}$. There were some aspects of the results which were difficult to understand in relation to connection/disconnection of other electrodes.

\section{Conclusions}

All the devices tested use currents below the limits $(100 \mu \mathrm{A}$ in normal condition and $500 \mu \mathrm{A}$ in single fault conditions) set for the safety of medical devices (IEC60601-1) with Type BF patient connections, which is the appropriate classification for biological amplifiers recording from the eye. Most of the items tested delivered currents which were an order of magnitude lower than the above, but the 1-2 $\mu \mathrm{A}$ limit recommended in the ISCEV Calibration Guidelines (2003), was not always achieved. Our measurements were continuous measurements, and could not take account of all possible transient currents arising from switching events.

\section{Poster B13}

\section{The insulating effect of silicon oil in vitrectomised human eyes}

Ajoy Vincent ${ }^{1}$, Rohit Shetty ${ }^{1}$, Naresh Kumar Yadav ${ }^{2}$, Priya B. Vijayan ${ }^{2}$, Sri Bhargav Natesh ${ }^{2}$, Bhujang Shetty ${ }^{1}$

${ }^{1}$ Department of Electrophysiology, Narayana Nethralaya, Super Specialty Eye Hospital and Post Graduate Institute, Bangalore, India; ${ }^{2}$ Department of Vitreoretina, Narayana Nethralaya, Super Specialty Eye Hospital and Post Graduate Institute, Bangalore, India

\section{Purpose}

To quantify the insulating effect of $1,000 \mathrm{CS}$ silicon oil in vitrectomised human eyes.

\section{Methods}

Prospective, longitudinal, non-randomized, case-control study undertaken after Institute ethical committee approval. Twenty-four eyes of 23 patients who underwent complete vitrectomy and silicon oil injection for intraocular tamponade following retinal detachment (RD) were included in the study. All the eyes had a bestcorrected visual acuity of $\geq 20 / 200$ on silicon oil and satisfied inclusion and exclusion criteria. A full-field ERG was done in accordance with ISCEV guidelines for both scotopic and photopic conditions. Full refractive correction at the testing distance was given for all the patients. Jet electrodes were used for the recordings. A combined maximal response was recorded under scotopic conditions with a standard flash $\left(1.5 \mathrm{~cd} / \mathrm{m}^{2}\right)$ and a dim light white scotopic response was recorded using a flash of $0.0083 \mathrm{~cd} / \mathrm{m}^{2}$. Most of the subjects also underwent a scotopic ERG with a flash of $0.08 \mathrm{~cd} / \mathrm{m}^{2}$. All the subjects then underwent a photopic ERG to the standard flash (surround luminosity of $30 \mathrm{~cd} / \mathrm{m}^{2}$ ) and a flicker ERG. Seventeen eyes of 17 patients then underwent repeat full-field ERG at one month following silicon oil removal (SOR). Three eyes of two patients are awaiting SOR over the next 4 weeks. The other eye of ten of these subjects that had no retinal abnormality served as control. Four patients who developed redetachment following SOR did not undergo repeat ERG. The dampening effect for each of the stimulus parameter was calculated as a ratio obtained for each value of the amplitude parameter.

\section{Results}

The mean values for different flash intensities for both photopic and scotopic conditions showed amplification by a factor $>2.5$ following SOR. The patients who later developed retinal detachment following SOR had mean ' $b$ ' wave amplitude to combined maximal response of $40.35 \mu$ volts with oil in situ. This was significantly lower than the mean value obtained in the group with silicon oil in situ who did not develop retinal detachment.

\section{Conclusions}

Silicon oil acts as a dampener to the intensity of light traversing the vitreous. The response to varying intensity of stimulus of full-field ERG gives an insight into the functional attachment of the outer retina at a given point in time when the eye has silicon oil in situ. It may be used as a prognosticator to predict the probability of RD following SOR. It may be used to assess the functional integrity of the outer layers of retina in cases that are lost to follow-up and present later with emulsified oil in the eye.

\section{Poster B14}

\section{A promising S-cone isolating protocol}

Josefin Nilsson $^{1,2}$, Thomas Wright ${ }^{1,2}$, Carol Westall ${ }^{2,3}$

${ }^{1}$ Department of Clinical Neurophysiology, Sahlgrenska Academy, Göteborg University, Sweden; ${ }^{2}$ Visual Electrophysiology Unit, The Hospital for Sick Children, Toronto, Canada; ${ }^{3}$ Department of Ophthalmology and Vision Sciences, University of Toronto, Canada

\section{Purpose}

Short-wavelength cones (S-cones) have been shown to be particularly vulnerable to retinal disease caused by systemic as well as ocular disorders. S-cone responses have accordingly been suggested as a sensitive marker of retinal disease allowing for earlier detection and possible intervention. Previously published 
S-cone ERG protocols give a response of only a few $\mathrm{mV}$, which has been reported as inadequate for clinical purposes. Developing a modified S-cone ERG protocol giving more practicable amplitudes would consequently be very valuable.

\section{Methods}

Ten adults with no oculo-visual defects acted as controls. In addition, four subjects with hereditary red-green colour defects and three patients with retinal disease were included in this study. ERGs were recorded using the Espion ColorDome system (Diagnosys LLC, Lowell, MA). To ensure adequate rod suppression, subjects were light adapted for 10 min using a white rodsuppressing background with an ISCEV recommended luminance previous to testing. Short-wavelength flashes (peak at $410 \mathrm{~nm}$ ) were obtained using a Wratten 47B filter (Kodak, Rochester, NY) in front of the ColorDome Xenon flash. The final S-cone response was averaged from 40 trials given at $2 \mathrm{~Hz}$, with trials containing obvious and/or large artefacts excluded at analysis.

\section{Results}

Short-wavelength flashes (peak at $410 \mathrm{~nm}$ ) of 80 photopic Td s/m², given on a $555\left(2.7 \mathrm{log} \mathrm{Td} / \mathrm{m}^{2}\right)$ photopic $\mathrm{Td} / \mathrm{m}^{2}$ long-wavelength background (peak at $594 \mathrm{~nm}$ ), produced a robust response in accordance with previously described S-cone wave morphology, demonstrating a small a-wave and a slow rising b-wave without any recordable OPs. Mean peak amplitude was found to be $46 \mu \mathrm{V}$ and mean peak latency $43 \mathrm{~ms}$. Responses from subjects with redgreen colour defects and from one subject with blue cone monochromatism demonstrated similar wave morphology and amplitude to that found in controls. One subject with Enhanced Scone Syndrome gave a supra-normal response, and another with $\mathrm{RP}$ gave a response with reduced amplitude. To validate further the response, several trial series including background and flash intensity series, rod recovery series and wavelength modifications of both background and stimulus, were performed.

\section{Conclusions}

We suggest a modified protocol with reduced background intensity, giving large amplitude S-cone responses, making marking and grading of the response more practicable. By using light adaptation previous to recording, rod intrusion is minimized. The suggested protocol, when added to the photopic section of a standard ERG, is $20 \mathrm{~s}$ long.

\section{Poster B15}

The assessment of the single-flash ERG a-wave slope by determination of derivatives in different time points

Sergey Rezvykh ${ }^{1,2}$, Irina Tsapenko ${ }^{1}$, Igor Spiridonov ${ }^{2}$, Marina Zueva ${ }^{1}$

${ }^{1}$ Moscow Helmholtz Research Institute of Eye Diseases, Moscow, Russia; ${ }^{2}$ Bauman Moscow State Technical University, Moscow, Russia

\section{Purpose}

The amplitude and peak latency of single-flash ERG a- and b-waves do not provide the accurate quantitative information about distribution of alterations in the retina. Only combined parameters of different kinds of ERG allow estimating the topography of alterations. The purpose of this work was a searching of new parameters to describe functional disorders by exploring the single-flash ERG and, first of all, parameters of the a-wave description.

\section{Methods}

Special software has been created, which provides the digital processing of ERGs (noise filtering, artifacts extraction), automatic finding characteristic points, and automatic determination of derivatives in different points. Software also permits to plot the distribution of derivative to view the development of these parameters in time. The curves of ERGs elicited to standard flash from 26 patients with proliferative diabetic retinopathy (PDR) and from 20 healthy subjects were used for analysis. We consider that the a-wave slope examination allows ascertaining disorders in early stages, and suggest estimating the speed of slope descending in this work. The measurement of derivatives in different time points up to $12 \mathrm{~ms}$ after the flash stimulus was carried out. For receiving values of derivative we apply nonrecursive differentiating filter with linear approximation by 3 points. Optimization criterion is the least square method. All signals are amplified in a band from 0 to $300 \mathrm{~Hz}$. Software and hardware filters reduce the high frequency noise level and the signal does not contain any artifacts. Therefore the linear approximation of filter is the most optimal for measuring derivatives. The algorithm of derivation is described in expression (1). The coefficients of filter are estimated by samples of single-flash ERG, where $T$-sampling time, $y_{i}$-samples of ERG:

$\frac{1}{10 T}\left\{-2 y_{-2},-y_{-1},+y_{1},+2 y_{2}\right\}$.

Results

The earlier part of a-wave slope is known to be a reflection of the photoreceptors' events, so it should be their quantitative description. We have determined the values of derivatives at 5,10 , and $12 \mathrm{~ms}$, respectively. At the time point $5 \mathrm{~ms}$, medians equaled $-8.7 \mu \mathrm{V} / \mathrm{ms}$ in normal and $-4.9 \mu \mathrm{V} / \mathrm{ms}$ in PDR. $25 \%$ and $75 \%$ percentiles equaled -4.5 and $-11.5 \mu \mathrm{V} / \mathrm{ms}$ for normal eye, respectively, and -0.9 and $-8.5 \mu \mathrm{V} / \mathrm{ms}$ - for pathology. At 10 and $12 \mathrm{~ms}$, medians decreased to -5.9 and $-2.3 \mu \mathrm{V} / \mathrm{ms}$ in normal, and -2.9 and -1.3 in PDR. Their percentiles had a substantial overlay. Therefore, the significant differences were obtained for time point parameters less than $10 \mathrm{~ms}$.

\section{Conclusions}

The software for determination of derivatives in different time points of ERG development has been created. Values of ERG a-wave slope derivatives were assessed, and the parameters earlier than $10 \mathrm{~ms}$ in the a-wave slope were shown to have the diagnostic significance in PDR. The future explorations will give us the knowledge of behavior of a-wave slope with others disorders.

\section{Poster B16}

\section{Software for the evaluation of electroretinograms}

Andre Messias, Herbert Jägle, Florian Gekeler, Eberhart Zrenner

Centre for Ophthalmology, University of Tuebingen, Tuebingen, Germany

\section{Purpose}

To develop a computer program to facilitate ERG response analysis, determining amplitudes and implicit times and OPs. 


\section{Methods}

Dephi 7 (Borland 2002) was used to create an application that runs in the Windows ${ }^{\circledR}$ operation system. This software version is dedicated to import the response text files generated with the Espion (Diagnosys LLC).

\section{Results}

After import, users can determine a- and b-wave amplitudes and implicit times by interactively defining the limits of the curve maxima and minima in all steps, single trials and channels of the given ERG protocol. OPs are obtained by applying a band-pass filter $(75-300 \mathrm{~Hz})$ using a Fast Fourier Transform (FFT) algorithm. Additionally, a Naka-Rushton function is fitted to a pre-selected set of amplitude responses to increasing flash intensity. All parameters are saved in a Paradox ${ }^{\circledR}$ data table for later analysis, re-use or export.

\section{Conclusion}

ERG evaluation is often a very time consuming task, particularly in trials that includes extended protocols. This system allows rapid evaluation of ERG parameters, as well as generating reports on a large amount of ERG data.

\section{Support}

The Tistou and Charlotte Kerstan Stiftung.

\section{Poster B17}

\section{Assessment of human dark adaption kinetic using the electroretinogam b-wave amplitude and dark adaptometry}

Susanne Kramer, Andre Messias, Eberhart Zrenner, Herbert Jägle

Centre for Ophthalmology, University of Tuebingen, Tuebingen, Germany

\section{Purpose}

To examine the kinetics of dark adaptation using Ganzfeld electroretinography (ERG) and psychophysical dark adaptometry.

\section{Methods}

ERGs were recorded with a Ganzfeld (Espion E2 system; Diagnosys LLC) using DTL electrodes. Normally sighted individuals were assigned to three groups according to the flash intensity used: $0.007 \mathrm{~cd} \mathrm{~s} / \mathrm{m}^{2}(n=10) ; 0.142 \mathrm{~cd} \mathrm{~s} / \mathrm{m}^{2}(n=19)$; and $3 \mathrm{~cd} \mathrm{~s} / \mathrm{m}^{2}(n=6)$. Measurements were performed before (pre-bleaching), and every $2 \mathrm{~min}$ after the exposure to intense illumination for $30 \mathrm{~min}$. Additionally, in 15 subjects psychophysical dark adaptation thresholds were obtained for a green and red target presented after an initial bleaching light in a Ganzfeld dome (Dark Adaptometer; Roland Consult).

\section{Results}

Following bleaching exposures (85\% and $93 \%$ cone photopigment bleaching for ERG and psychophysics, respectively) the ERG response amplitude followed a sigmoid function. B-wave amplitude was kept below ca. 20\% (for dimmest flash, 30\% and $40 \%$ for brighter flashes) of pre-bleaching for about $5 \mathrm{~min}$ and then recovered at a rate of ca. $10 \%(6 \%$ and $5 \%$ for brighter flashes) per minute. After ca. $20 \mathrm{~min}$ b-wave amplitude reaches saturation level regardless the flash intensity. Psychophysical dark adaptation thresholds showed a monophasic (red stimulus) or biphasic (green stimulus) course of cone and rod adaptation. On average, the cone-rod break
(Kohlrausch kink) was reached after ca. $10 \mathrm{~min}$ and the final rod threshold after ca. $28 \mathrm{~min}$. Cone thresholds estimated from the red and green curves were similar.

\section{Conclusion}

While a comparison of both methods is difficult due to a variable contribution of cones and rods to ERG responses, both allow inferring information about the kinetics of dark-adaptation. The methods are applicable for clinical trials dealing with disorders of the visual cycle or toxicology studies on substances that are suspected to affect the retinal pigment epithelium.

\section{Support}

Grant JA997/5-1 of the German Research Council.

\section{Poster B18}

The photopic negative response of the red on blue electroretinogram using led built-in contact lens electrodes on open angle glaucoma

Kaoru Fujinami ${ }^{1,2}$, Rishu Inoue ${ }^{2}$, Toshine Maeda ${ }^{2}$, Toyoko Inoue $^{2}$, Yoichi Inoue ${ }^{2}$, Kazushige Tsunoda ${ }^{1}$, Kei Shinoda ${ }^{3}$, Yozo Miyake ${ }^{4}$

${ }^{1}$ The National Institute of Sensory Organs, National Tokyo Medical Center, Tokyo, Japan; ${ }^{2}$ Olympia Eye Hospital,

Tokyo, Japan; ${ }^{3}$ Oita University, Oita, Japan;

${ }^{4}$ Aichi Shukutoku University, Aichi, Japan

\section{Purpose}

The photopic negative response (PhNR) of the photopic ERG recorded using a Ganzfeld stimulation was reported to correlate with visual field sensitivity losses in primary open angle glaucoma (POAG) by Viswanathan et al. (IOVS 2001). The present study was conducted to determine whether the PhNR recorded using light emitting diodes (LED) built-in contact lens electrodes correlates to visual field sensitivity losses and retinal nerve fiber thickness (RNFT) in patients with POAG.

\section{Methods}

Sixty-nine eyes with POAG were examined. The mean age was 54.3 years (range, 31-77 years). Full-field ERGs were recorded with LED built-in contact lens electrodes. The wavelength, strength, and duration of the flash were $644 \mathrm{~nm}$ (red), $891.2 \mathrm{~cd} / \mathrm{m}^{2}$, and $3 \mathrm{~ms}$, respectively. The wavelength and strength of steady background illumination were set as $470 \mathrm{~nm}$ (blue) and $10 \mathrm{~cd} / \mathrm{m}^{2}$ in order to saturate the rods. Thirty-two responses were summed with a stimulus frequency of $2 \mathrm{~Hz}$. Visual field sensitivities and RNFT were measured with the automated static perimetry (Octopus 500 program 32) and optical coherence tomography (OCT, Stratus OCT model 3000) software, respectively.

\section{Results}

The PhNR amplitude was significantly $(r=0.43 P<0.05)$ correlated with mean deviation (MD) losses ranging from -3.7 to $24.7 \mathrm{~dB}$. It was also significantly $(r=-0.49 P<0.05)$ correlated with RNFT ranging from 34.1 to $124.1 \mu$. Namely, POAG eyes with lower PhNR amplitude showed more severe MD loss and thinning of RNFT.

\section{Conclusion}

The PhNR recorded with LED built-in contact lens electrodes reflected visual sensitivity and morphological changes in POAG eyes. It may be a good indicator for disease severity, thereby being useful for monitoring the glaucomatous damage. 


\section{Poster B19}

\section{Investigation of photopic negative response in glaucoma} by focal electroretinograms

Hajime Nakamura, Masanori Hangai, Fumitaka Hirose, Tomonari Ojima, Nagahisa Yoshimura

Department of Ophthalmology and Visual Sciences, Kyoto University Graduate School of Medicine

\section{Purpose}

To investigate the photopic negative response (PhNR) of glaucoma patients using focal macular ERG and to evaluate the association between PhNR and severity of visual field defect of eyes with glaucoma.

Methods

PhNR of focal macular ERG was recorded from 29 eyes of 29 patients with glaucoma (age, $60.1 \pm 13.2$ years). Focal macular ERG was elicited by 15 degree visual angle spotlight positioned on the fovea and monitored by an infrared fundus camera. The amplitude of the PhNR was compared with 54 normal controls (age, $53.2 \pm 21.8$ years). The patients of glaucoma were also recorded PhNR of full-field ERG and measured static perimetry and Matrix FDT perimetry. We evaluated the relationship between the PhNR amplitude of focal macular ERG and fullfield ERG and the mean deviation (MD) and pattern standard deviation (PSD) of static perimetry and Matrix FDT perimetry.

\section{Results}

The mean PhNR amplitude ( \pm S.D.) was $0.8 \pm 0.7 \mu \mathrm{V}$ for glaucoma patients and $2.5 \pm 0.6 \mu \mathrm{V}$ for normal controls. Glaucoma patients showed significantly small amplitude in PhNR than normal controls $(P<0.001)$. The MD and PSD was $-7.7 \pm 6.4 \mathrm{~dB}$ and $8.5 \pm 5.0 \mathrm{~dB}$ in static perimetry and $-9.2 \pm 5.7 \mathrm{~dB}$ and $6.5 \pm 2.7 \mathrm{~dB}$ in Matrix FDT perimetry in glaucoma patients, respectively. There was a significant correlation in focal macular ERG between the PhNR and the MD and PSD of static perimetry $(P<0.05, R=0.41$ and $P<0.005$, $R=-0.52)$ and Matrix FDT perimetry $(P=0.001, R=0.56$ and $P<0.5, R=-0.38$ ), but not in full-field ERG.

\section{Conclusions}

These findings indicate that the PhNR amplitude of focal macular ERG evaluates the functional impairment of retinal ganglion cells qualitatively and quantitatively in glaucoma patients.

\section{Poster B20}

\section{Microcurrent neuromodulation in Retinitis Pigmentosa: report of a pilot study}

Subhadra Jalali, Raja Narayanan

Smt. Kanuri Santhamma Retina Vitreous Centre, LV Prasad Eye Institute, Hyderabad, India

\section{Purpose}

To describe the clinical and electrophysiological changes after use of investigational microcurrent neuromodulation $(\mathrm{MCN})$ in a pilot study for eyes with Retinitis Pigmentosa (RP).

\section{Methods}

20 patients (40 eyes) of RP with visual acuity less than 20/40 but not worse than 20/200 were recruited in this interventional pilot study. RP was defined by signs and symptoms including bilateral, progressive visual loss, peripheral field loss (less than 30 degrees but more than 5 degrees), nyctalopia, diffuse widespread RPE degeneration with generalized arterial narrowing and Flash ERG Scotopic b wave less than 50 microvolts. Microcurrent neuromodulation (MCN) of the eyes was done as per protocol algorithm twice daily for $20 \mathrm{~min}$ during the period of study using ScyFIX $650{ }^{\mathrm{TM}}$ MCN Device. Patients could continue Vitamin A supplementation along with the device. Investigations were done at baseline, 1, 3, 6 and 12 months. Investigations included: Best-Corrected visual acuity (log MAR), Complete ophthalmic evaluation, OCT, Fluorescein angiography, Flash and Multifocal ERG, Contrast sensitivity, automated perimetry 30-2 and 10-2, and Visual Function Questionnaire (VFQ). Any adverse events were noted. Study protocol was approved by Institutional Ethics committee.

Results

Mean age of the 16 males and 4 females was 31 years (range 20-52 years). Median follow-up was 6.5 months ranging from 2-12 months. Device stability, acceptance and compliance were good with only 2 events of protocol deviation and one instance of device malfunction reported. Mild to moderate headache was reported in $3 / 20$ subjects. No subject discontinued the trial. Results of patients completing 6 months are reported. At 6 months, contrast sensitivity in 24 eyes showed mean improvement of 2 grating acuity at high $(1.5 \mathrm{cpd})$ contrast grating acuity, while the low contrast grating acuity did not change. In visual fields, the mean deviation improved by 4 points at 6 months. Mean corrected near vision improved by 1.4 lines and distance vision by 1.3 lines. On VFQ, 8 of the 10 patients who completed 6 months questionnaire had improved VFQ scores ranging from 1 to 4 points for a mean of $8 / 14$ questions (range 3-12 questions). Improvement was significant for near tasks and tasks in dim illumination. Corresponding changes in Flash ERG and multifocal ERG as expected at this stage were minimal and were difficult to interpret.

\section{Conclusion}

Microcurrent neuromodulation of eyes with moderate Retinitis Pigmentosa demonstrated device stability, patient acceptance and safety in this pilot study. Subjective improvement for near tasks and tasks in dim illumination was recorded. Minor improvements in visual fields, contrast sensitivity and near vision seen over this very short study period need to be studied over a longer period of time.

Support

Trial supported by ScyFIX

\section{Poster B21}

The leber congenital amaurosis protein, Aipl1, plays a direct role and is indispensable for cone photoreceptor cells

Lindsay Michalovicz, Saravanan Kolandaivelu, and Visvanathan Ramamurthy

West Virginia University Eye Institute, Morgantown, WV 26506-9193

\section{Purpose}

Leber congenital amaurosis (LCA) is a retinal degenerative disease that causes blindness in children. Several mutations in 
Aryl hydrocarbon Receptor Interacting Protein Like -1 (Aipl1) are linked to a severe form of LCA. A mouse model lacking AIPL1 recapitulates the disease showing no visual activity accompanied by rapid degeneration of both rod and cone photoreceptors. Rod photoreceptor death is due to the absence of stable rod phosphodiesterase (PDE), an enzyme essential for rod function and survival. However, the reason behind cone photoreceptor degeneration and the lack of cone function in both humans with LCA and our mouse model remains unclear.

\section{Methods}

Rapid cone cell death possibly results from an indirect effect of rod cell death, due to the high rod:cone ratio (97:3) in the mouse retina, or a direct and essential role for AIPL1 in cone cells. To differentiate between these two models and to understand the role of AIPL1 in cones, we used a transgenic approach to create a mouse where AIPL1 is exclusively expressed in rod photoreceptors and not in cone photoreceptors. We find that cone photoreceptors lacking AIPL1 degenerate slowly over time in the presence of functional rod photoreceptors, similar in time scale to dominant cone dystrophies. This is in contrast to AIPL1 knockouts where cones degenerate rapidly when accompanied by rapid rod photoreceptor degeneration.

\section{Results}

Dark-adapted electroretinography (ERG) shows that the transgenic expression of AIPL1 rescues rod function. However, light-adapted ERG, isolating cone photoreceptor function, shows no response in the absence of AIPL1. This lack of cone response demonstrates that AIPL1 is essential for proper cone functioning despite normal cone photoreceptor morphogenesis. Western blot analysis reveals that the lack of cone response seen in the absence of AIPL1 coincides with reduced levels of cone PDE. Altogether, our results demonstrate that AIPL1 is necessary for the functioning and survival of cone photoreceptors.

\section{Conclusions}

In agreement with previous work, our study also shows that the maintenance of cone photoreceptors relies on functional rod photoreceptors. In concert with previous findings regarding AIPL1's role in rod photoreceptors, elucidating the function of this protein in cone photoreceptors allows for a better understanding of the pathology seen in LCA patients.

\section{Poster B22}

\section{Treatments of neovascular age-related macular degeneration: a quantitative review}

J. Vernon Odom ${ }^{1}$, Ahmed El-Sherbeeny ${ }^{2,3}$, Nabil Jabbour ${ }^{1,2}$

${ }^{1}$ West Virginia University Eye Institute, Morgantown, WV;

${ }^{2}$ Mid Atlantic Retina Consultations, Morgantown, WV;

${ }^{3}$ Al-Jouf University, Saudi Arabia; ForSight Foundation, Morgantown, WV

\section{Purpose}

To conduct a quantitative review (meta-analysis) of the effectiveness of different treatments for Neovascular AgeRelated Macular Degeneration (NAMD) to compare monotherapy (photodynamic therapy [PDT] with verteporfin, antivascular endothelial growth factor treatments [Anti-VEGF; Lucentis and Avastin], and steroids [Kenalog]) and combination therapies (PDT and Avastin; PDT and Lucentis) with respect to visual acuity (logMAR) improvement and total number of treatments per year.

\section{Methods}

We surveyed the literature up to July 2007 using the PubMed database and ARVO abstracts and selected those articles and abstracts which provided quantitative numbers regarding acuity improvement and number of treatments. If the number of treatments was expressed for a period shorter or longer than one year, the treatments were expressed as total number of treatments per year.

Results

Our results are presented in the following table.

\begin{tabular}{lllll}
\hline Mean & Mean & Mean & Mean & Total \\
base & final & logMAR & ETDRS & treatments/ \\
logMAR & logMAR & gain & $\begin{array}{l}\text { letter } \\
\text { gain }\end{array}$ & year \\
& & & gin & \\
\hline
\end{tabular}

\begin{tabular}{lccccc}
\hline \multicolumn{2}{l}{ Non-VEGF treatments } & & & & \\
PDT & 0.653 & 0.891 & -0.239 & -11.933 & 2.589 \\
Kenalog & 0.823 & 0.838 & -0.011 & -0.558 & 2.629 \\
PDT + & 0.861 & 0.882 & -0.023 & -1.132 & 3.342 \\
$\quad$ Kenalog & & & & & \\
Anti-VEGF treatments & & & & \\
Lucentis & 0.775 & 0.689 & 0.086 & 4.320 & 10.842 \\
Avastin & 0.920 & 0.789 & 0.134 & 6.697 & 7.086 \\
PDT + & 0.687 & 0.532 & 0.135 & 6.738 & 11.013 \\
$\quad$ Lucentis & & & & & \\
PDT + & 0.937 & 0.801 & 0.139 & 6.928 & 6.853 \\
$\quad$ Avastin & & & & & \\
\hline
\end{tabular}

\section{Conclusions}

Treatments which administer anti-VEGF result in greater visual acuity improvements than previous treatments of NAMD. Although treatments which involve combining PDT with Anti-VEGF are promising, as a group they have yet to demonstrate a clear advantage over Anti-VEGF treatments alone either in terms of visual acuity improvements or in terms of the total number of treatments required in a year. The present analysis remains limited because of the limited number of combination studies, uncertain criteria for who might benefit from combination therapy, and preferred treatment protocols. Support

EY 14841 from the National Eye Institute, Mid-Atlantic Retina Consultations, Inc. and the ForSight Foundation. 


\section{Oral Presentation 7: Retinal Structure And Function}

Chairs: Jonathan S. Lyons \& Masayuki Horiguchi

\section{Invited Lecture 3: Low vision care and electrophysiology: from a clinical point of view}

\section{Kenji Yanashima}

Kitasato University, School of Allied Health Sciences, Sagamiharashi, Kanagawa, JAPAN; Yanashima Eye Clinic, Kamata, Tokyo, Japan

What happens in the visual cortex of the visually impaired? An alteration of the visual center was examined using MRS (Magnetic Resonance Spectroscopy). NAA ( $N$-acetyl aspartate) is a compound which exists in neurons and is a useful indicator of neural function. The level of NAA in neurons decreases with neural damage and in the visual cortex is found to be statistically lower in people with visual impairment than in those with normal vision. Conversely, higher levels of $\mathrm{Cr}$ (creatine) and Cho (cholesterol) are found in the same area of the visually impaired. These must be considered in the further research and development of artificial retina.

Many medical professionals believe that successful diagnosis and treatment of patient ailments are the primary responsibilities of those involved in health care. However, patients with visual impairment expect not only medical therapy but also consultation on how to deal with the effects their ailments and their associated treatments have on their daily lives. Because many ophthalmologists concentrate only on the diagnosis and treatment of eye disease and not on these day-to-day difficulties, many patients have expressed dissatisfaction with the care that they have received. Low vision care aims to alleviate this dissatisfaction. Low vision care is patientoriented treatment-therapy based on and tailored to the individual needs of each patient—and it encompasses medical diagnosis and treatment as well as counseling on how to cope with visual impairment. Low vision care aims to elevate the quality of life of patients to its original level, the one they had before they suffered from their respective diseases. Before starting low vision care services, accurate diagnosis and prognosis are of the utmost importance.

Electrophysiological examination is essential, especially in differential diagnoses of macular diseases. Low vision care is also critical in the effective treatment of patients.

\section{OS7-Oral Paper 1}

Objective assessment of retinal functions of subjects with advanced retinal degeneration in clinical trials

R. Wilke ${ }^{1}$, M. Bach ${ }^{2}$, A. Schatz ${ }^{1}$, T. Strasser ${ }^{1}$, H. Benav ${ }^{1}$, A. Messias ${ }^{1}$, E. Zrenner ${ }^{1}$

${ }^{1}$ Institute for Ophthalmic Research, Department of Ophthalmology, University of Tübingen, Germany, ${ }^{2}$ University Eye Hospital Freiburg, Freiburg, Germany

\section{Purpose}

Trials using novel therapeutical approaches, like gene therapy or artificial vision devices, will rely on the inclusion of subjects with highly reduced retinal functions. This is a challenge, as standard methods to assess visual functions tend to fail there. We present a method to selectively assess heavily impaired rod and cone functions using refined ERG methods.

\section{Methods}

Recordings were performed on an e2 espion system with a color dome LED stimulator. To identify very small rod and cone functions, a 3-step approach was used: (1) For isolation of rod dominated ERGs, blue $(470 \mathrm{~nm})$ or green $(513 \mathrm{~nm}) 9-\mathrm{Hz}$ stimuli of 6-14 ms duration were used; for cone isolation a red $33-\mathrm{Hz}$ flicker stimulus $(635 \mathrm{~nm}, 8-14 \mathrm{~ms})$ was applied. 75 individual sweeps were averaged. (2) Offline analysis: Detrending using subtraction of Lorentzian minimization fit of 3rd order polynomials and consecutive Fourier analysis (Singleton's Mixed Radix FFT) were used to extract the signal. (3) Signal-to-noise assessment: AR modeling of every individual recording was used to assess the noise level at the expected frequency band of the signal. A Monte-Carlo approximation was applied to calculate peak-based critical limit significance levels of the extracted signal in respect to underlying noise.

\section{Results}

By recording V-log-I responses in 32 eyes of healthy volunteers we found a blue stimulus of $0.012 \mathrm{scot} \mathrm{cd} \mathrm{s} / \mathrm{m}^{2}$ and $10 \mathrm{~ms}$ duration to yield maximum rod-pathway specific responses. For mixed L- and M-cone specific responses, intensities of $0.12 \mathrm{~cd} \mathrm{~s} / \mathrm{m}^{2}$ with $10 \mathrm{~ms}$ duration were determined. For the rod response, dark adaptation recovery after bleaching showed initial elimination of responses after bleaching, followed by regular adaptation kinetics, indicating that the chosen stimulation protocol does not interfere with the dark adapted state and addresses mainly rod function. A full set of normative values has been established. In 128 of 207 recordings of patients with undetectable standard ISCEV rod response (visual acuity between light perception and 20/20) a 9-Hz rod flicker response could be measured which exceeded the $95 \%$ confidence limit against noise. Signals of less than $0.5 \mu \mathrm{V}$ can thus be measured reliably.

\section{Conclusions}

This method allows for the objective detection of very small remaining retinal ERG responses even if standard clinical measures like visual field, visual acuity, and ISCEV standard ERG protocols fail. This method may be a valuable tool in clinical trials where legally blind persons will be included.

Support

European Union Integrated Project EVI-GENORET LSHGCT-2005-512036.

\section{OS7-Oral Paper 2}

Clinical and electrophysiologic characteristics of a large kindred with X-linked Retinitis Pigmentosa associated with the RPGR locus

Byron L. Lam ${ }^{1}$, Audina Berrocal ${ }^{1}$, Tania Arguello ${ }^{1}$, William Feuer ${ }^{1}$, Mu Liu ${ }^{1}$, Ditte Hess ${ }^{1}$, Michelle Caputo ${ }^{1}$, Maria Berrocal $^{2}$, Alexandra Weisman ${ }^{2}$, Edwin M. Stone ${ }^{3}$ 
${ }^{1}$ Bascom Palmer Eye Institute, University of Miami, USA;

${ }^{2}$ Berrocal Eye Clinic, Puerto Rico, USA; ${ }^{3}$ University of Iowa Carver College of Medicine, USA

\section{Purpose}

To phenotypically and genotypically characterize a large Puerto Rican kindred with X-linked Retinitis Pigmentosa (RP) associated with the RP GTPase regulator (RPGR) genotype.

\section{Methods}

100 family members of a Puerto Rican kindred with X-linked RP were evaluated with comprehensive ophthalmic examinations and blood DNA analysis. Visual fields, fundus photographs, Stratus OCT3, and standard full-field ERG were obtained on all affected males, all obligatory carriers, and all possible carriers as determined by pedigree.

\section{Results}

Of the 100 family members, 13 were affected males and 18 were carriers. The mean age of the 13 affected males was 37 years (range 9-70), and the mean age of onset of visual symptoms was 12 years (range 5-30). Best eye visual acuity was highly correlated with age (Spearman coefficient $=0.95)$, with hand-motion acuity by age 35 and light perception to no light perception by age 50-60. Visual fields were minimally plottable by age 40, and standard full-field ERG responses reached non-detectable levels by late teens. The mean age of the 20 carriers was 29 years (range 1-66). The carriers had no or mild visual symptoms and the median visual acuity of the carriers was 0.8 with no significant change with age. All carriers had a visual acuity of at least $20 / 50$ or better in one eye, and the amount of pigmentary retinal degeneration was highly variable, with ERG responses ranging from severely impaired to near normal. Virtually all carriers were identifiable with a combination of clinical and ERG examinations. DNA analysis suggests RPGR genotype with no mutation in exons $1-14$ of the RPGR gene; screening of the ORF15 portion of the RPGR gene is in progress.

\section{Conclusions}

Profound visual loss and extinguishing ERG occurred by the second decade of life with progression to near no light perception by age 60 in this large kindred of X-linked RP associated with the RPGR genotype. Female carriers maintained visual acuity with age and were identifiable by clinical and ERG examination. The information from this study is important to determine the optimal age for intervention as new RP treatments are being developed and tested.

\section{OS7-Oral Paper 3}

\section{Meeting the challenge of reliable electrophysiological retinal function testing in multi-centre trials: state of development of the "iscev protocols for clinical trials"}

E. Zrenner (for the ISCEV-Committee on ISCEV protocols for clinical trials)

Centre of Ophthalmology, University of Tübingen, Germany

\section{Purpose}

To line out new developments-fostered by ISCEV — that help to improve the quality of multi-centre safety and efficacy trials by ISCEV-approved standard operating procedures (SOPs) that propose stringent parameters for stimulation and recording based on the ISCEV standards.

\section{Background}

In recent years, electrophysiological non-invasive testing has increasingly become important in pre-clinical and clinical studies, concerning efficacy and safety as well as identification of the possible origin of drug-induced pharmacological or toxicological effects in the visual system. Proper standards for all tests, EOG, ERG, multi-focal ERG, pattern ERG, VEP as well as calibration procedures are available (see http://www. iscev.org). Nevertheless the variation of responses across centres is still much too large as to allow easy performance of multi-centre trials. Moreover, the design of multi-centre studies requires special expertise in order to select the proper endpoints on the basis of the particular mechanisms of drug action, retinal circuitry and biochemistry. Quality has to be ensured by detailed, purpose-oriented standard operating procedures and case report forms that should make multi-centre electrophysiological testing in the eye much easier in terms of quality, quality control, central reading and monitoring as well as data management. Therefore, ISCEV in 2007 asked a committee to develop ISCEV protocols for clinical trials.

\section{Present state of discussion}

$V$-log I functions: The ISCEV standard in the dark adapted state asks for two responses, the combined rod-cone response, elicited with the standard flash $\left(1.5-3 \mathrm{cds} / \mathrm{m}^{2}\right)$ and the rod response, elicited with the standard flash, attenuated by $2.5 \mathrm{log}$ units. These are just two points that relate the voltage of the amplitude to the luminance of the Ganzfeld bowl. The quality of the recordings is greatly improved, if this function (V-log Ifunction) is not only determined by 2 points but at least by a series of stimuli of increasing luminance because each result contributes to this function. Outliers are not heavily influencing the shape of the function and additionally sensitivity can be assessed independently of amplitude variations.

Increased sensitivity in small signal recordings: Trials using novel therapeutic approaches often rely on the inclusion of persons with very reduced retinal functions for safety and efficacy issues. This is a challenge as standard methods tend to fail. Recommendations are developed to separately assess "Submicrovolt Ganzfeld Recordings" for rod and cone pathways.

Visual Cycle Kinetics: For the rod response, dark adaptation recovery after bleaching shows initial elimination of responses, followed by regular adaptation kinetics, indicating that the chosen stimulation protocol does not interfere with the dark adapted state and addresses mainly rod function.

Separation of spectrally different cone pathways: Separation of $\mathrm{L}$ - and M-cone specific responses and S-cone specific responses requires certain characteristics of stimulation and adaptation to assess the particularly vulnerable $\mathrm{S}$-cone function.

ON-OFF pathway assessment: Most effective settings shall be determined in order to differentiate these pathways that show different sensitivity to various compounds.

\section{Conclusion}

The development of ISCEV-approved protocols will be presented and discussed. Detailed protocols for use of electrophysiological endpoints for a series of particular trial conditions shall help to improve the quality of multi-centre studies by proper standard operating procedures and stringent case report forms, based on ISCEV standards and recommendations. 


\section{Oral Session 8: Imaging and visual electrophysiology}

Chairs: Marko Hawlina \& Colin Barber

\section{Invited Lecture 4: Inner retinal sequelae of outer retinal disease: analysis of ERG components in the RCS rat}

\author{
Paul A. Sieving, Ronald A. Bush, Dorit Raz, \\ Shigeki Machida
}

National Eye Institute, NIH, Bethesda, MD

The ERG of the Royal College of Surgeons (RCS) rat undergoes considerable change in the waveform during progressive degeneration of the photoreceptors in the outer retina. Under dark-adapted recording conditions, the scotopic threshold response (STR) persists into late stages of degeneration in advanced age. This negative response originates in the proximal retina and comes to dominate the ERG despite severe reduction of the scotopic b-wave.

Cone photoreceptors and cone-driven visual function persists into late stage RCS disease, even beyond the loss of rods and recordable rod ERG components. The photopic ERG at this stage is dominated by a negative waveform component despite major reduction of the photopic b-wave. Application (through intra-vitreal injection) of agents that block transmission primarily at sites in the inner retina suppress these negative photopic responses (NPR) of the RCS retina and uncover considerable residual b-wave that presumably was previously swamped by the negative waveform.

We have explored the origins of these negative ERG components of the RCS rat and their relationship to potassium currents in the proximal retina. One lesson learned is that the usual rules for ERG interpretation were developed from study of normal retinal function, and these rules may well be altered by retinal disease.

\section{OS8-Oral Paper 1}

Simultaneous recording of near-infrared spectroscopy and visual evoked potentials in normal human subjects

Kazuhiko Tanabe ${ }^{1}$, Atsushi Mizota ${ }^{1}$, Minoru Tanaka ${ }^{1}$, Ichiro Shimoyama ${ }^{2}$

${ }^{1}$ Department of Ophthalmology, Juntendo University

Urayasu Hospital, Urayasu, Japan; ${ }^{2}$ Human

Neurophysiology, Research Center for Frontier Medical

Engineering Chiba University, Chiba, Japan

\section{Purpose}

Near-infrared spectroscopy (NIRS) is a method to measure dynamic changes in hemoglobin concentration evoked by brain activity. We recorded NIRS and VEP simultaneously with pattern stimulus. The purpose of the present study is to investigate the relations between NIRS and VEP to pattern stimulus.

\section{Methods}

We recorded NIRS and VEPs simultaneously with both pattern reversal hemifield and full-field stimulus in healthy volunteers. For recording of NIRS, we used a near-infrared spectroscopy system (FOIRE-3000, Shimadzu Corporation, Kyoto, Japan) with wave length of 780,805 , and $830 \mathrm{~nm}$ to monitor changes in the oxyhemoglobin, deoxyhemoglobin, and total hemoglobin, respectively, with 48 channels over the occipital lobe. VEPs was recorded with positive electrodes placed at $\mathrm{O} 1$ and O2. Black and white checker-boards on a CRT monitor were used as stimuli. A check size was $1.7^{\circ}$ and reversal rate was 6 reversal/second. We recorded with 3 stimulus fields, right and left hemifields and full field. Every $20 \mathrm{~s}$ the checker-boards were altered right and left hemifield and full field, with resting period of $20 \mathrm{~s}$.

\section{Results}

In VEPs, clear responses were observed in contralateral occipital lobe with hemifield stimulus and both occipital lobe with full field stimulus in all cases. But in NIRS, neither significant increase in oxyhemoglobin nor decrease in deoxyhemoglobin was observed in several cases.

\section{Conclusion}

Both VEPs and NIRS can reflect the activity of occipital lobe to pattern reversal stimulus, but the sensitivity might be higher for VEPs in the present conditions.

\section{OS8-Oral Paper 2}

Association between functional markers and structural retinal changes in adolescents with type 1 diabetes

Ekta Lakhani $^{1,2}$, Thomas Wright ${ }^{2}$, Giuseppe Mirabella ${ }^{2}$, Peter Glazer $^{2}$, Carol Westall ${ }^{1,2}$

${ }^{1}$ The Institute of Medical Science, University of Toronto; ${ }^{2}$ Department of Ophthalmology and Vision Sciences,

The Hospital for Sick Children

\section{Purpose}

(1) To use electrophysiological measures to detect retinal changes associated with type 1 diabetes and (2) to correlate spatially areas of neuronal dysfunction in the retina with areas of vascular abnormalities associated with diabetic retinopathy. Methods

To date, 35 patients (12-20 years old) that have had type 1 diabetes for at least 5 years and 20 healthy age-similar controls have undergone electrophysiological testing using the standard (mfERG) and slow-flash (sfERG) multifocal electroretinograms. MfERG (103 hexagons) allowed assessment of neuronal function in outer and middle retinal layers and sfERG (61 hexagons) allowed assessment of neuronal function in the inner retina. Ranges of normal times and amplitudes of response waveforms per hexagon were determined using $95 \%$ confidence intervals of control data. This allowed calculation of the number of abnormal hexagons per subject. Patients also underwent fundus photography in order to determine the presence or absence of diabetic retinopathy. A subsample of patients and controls $(n=20)$ will undergo the short-wavelength ERG (sERG) to assess S-cone function. In addition, a subsample of patients $(n=5)$ with various degrees of diabetic 
retinopathy will undergo retinal imaging using the adaptive optics confocal laser ophthalmoscope-ultra-high-resolution optical coherence tomograph.

\section{Results}

mfERG results showed no significant differences in the mean number of abnormal hexagons for amplitudes (controls $4 \pm 7$, patients $5.5 \pm 8$ ) or times (controls $0.5 \pm 0.8$, patients $0.5 \pm 1.2$ ) between the two groups. A qualitative assessment of the sfERG waveforms showed a prominent decrease in amplitudes and increase in times for the patient group in comparison to controls.

\section{Conclusions}

Preliminary sfERG results suggest inner retinal dysfunction in patients with type 1 diabetes. Future research will determine whether these electrophysiological results predict vascular abnormalities identified from retinal fundus images. Future work will also evaluate neuronal changes at the cellular level and correlate them with areas of functional abnormality. This exploratory investigation will be performed using adaptive optics technology, which will allow us to obtain highresolution retinal images by correcting aberrations caused by the eye's cornea and lens.

\section{OS8-Oral Paper 3}

\section{Sensitivity and specificity of perg in relation to stimulus field size and OCT in patients with Stargardt disease}

Eva Lenassi, Darko Perovšek, Martina Jarc-Vidmar, Petra Popovič, Marko Hawlina

Eye Clinic, University Medical Centre, Ljubljana, Slovenia

\section{Purpose}

PERGs provide information about macular function and they are therefore important for diagnosis and management of maculopathies, such as Stargardt disease. The aim here was to investigate the influence of the stimulus field on the PERG amplitude, and its sensitivity and specificity for evaluating macular function in Stargardt disease, while also investigating the relationship between PERG and optical coherence tomography (OCT) in patients with genetically confirmed Stargardt disease.

\section{Methods}

The PERGs to standard $\left(12^{\circ} \times 16^{\circ}\right)$ and larger $\left(24^{\circ} \times 32^{\circ}\right)$ stimulus fields were recorded in 10 control subjects and 10 patients with Stargardt disease. In the latter, full ophthalmological examination, OCT (Topcon 3D OCT-1000), autofluorescence imaging (Heidelberg Engineering HRA) and fundus-related perimetry (Nidek Technologies MP1 Microperimeter) were performed. The PERGs were recorded with a Roland Consult RetiScan using HK-loop electrodes.

\section{Results}

In control subjects, the PERG P50 amplitude was significantly higher $(P<0.05)$ with larger stimulus field $(5.0 \pm 0.7 \mu \mathrm{V})$ in comparison to standard stimulus field $(2.6 \pm 0.4 \mu \mathrm{V})$. Also, in patients with Stargardt disease, the PERG P50 amplitude was significantly higher $(P<0.05)$ with larger stimulus field $(0.5 \pm 0.4 \mu \mathrm{V})$ in comparison to standard stimulus field $(1.9 \pm 1.6 \mu \mathrm{V})$. For the PERGs to standard stimulus field, a cut-off criterion of $1.8 \mu \mathrm{V}$ represented $100 \%$ specificity and
$100 \%$ sensitivity. With PERGs to larger stimulus field, the cutoff criterion was higher $(4.2 \mu \mathrm{V})$ and resulted in $91 \%$ specificity and $91 \%$ sensitivity. Stargardt patients had varying central atrophy in the autofluorescence imaging and transverse loss of the central photoreceptor layer in the OCT. The PERG amplitude increase with larger stimulus field was seen in patients with the lesser transverse photoreceptor loss in OCT, while there was no increase in PERG amplitude with larger stimulus field in patients with severe transverse photoreceptor loss.

\section{Conclusion}

In patients with Stargardt disease, the sensitivity and specificity of PERGs to larger stimulus field were lower in comparison to standard stimulus field, but most of the PERGs to standard field were non-recordable. The increase in PERG amplitude to larger stimulus field was seen to correlate with the severity of transverse photoreceptor loss in OCT. These findings suggest that PERGs to larger stimulus field might provide important evaluations of the extent of photoreceptor damage in patients with Stargardt disease.

\section{OS8-Oral Paper 4}

\section{Multimodal imaging: a new tool to investigate the relationship between macular structure and function in retinal disease}

\section{S. M. Walker, S. Parks, D. Keating}

Tennent Institute of Ophthalmology, Gartnavel General Hospital, Glasgow, UK

\section{Purpose}

To investigate the effects of retinal disorders with macular involvement on macular structure and corresponding function using multimodal imaging (MMI), a new imaging modality which combines a scanning laser ophthalmoscope, optical coherence tomography and micro-multifocal ERG (micromfERG).

\section{Methods}

A prototype OCT/SLO system (OTI; Toronto, Canada) was modified to integrate an organic light emitting diode (OLED) display into the optics of the system. A high resolution micromfERG stimulus covering up to the central 24 degrees was projected onto the OLED display. During the 8-min micromfERG recording time, OCT scanning was performed in both coronal (C-scan) and transverse (B-scan) planes in multiple locations to allow structural and functional information to be collected for all the stimulated areas. Accurate patient fixation during the recordings was ensured by monitoring the SLO channel. 55 patients with conditions affecting the macula (14 with a macular hole, 25 with age-related macular degeneration and 16 with other conditions) have been assessed using MMI and the structural and functional information obtained has been correlated. 12 patients with a macular hole and 15 patients with age-related macular degeneration (ARMD) have been reassessed with MMI post-treatment and the structural and functional changes observed.

\section{Results}

MMI was well tolerated by all patients tested with good patient compliance observed in all recordings. Good correlation was observed between structural and functional alterations in most 
patients although in some cases functional abnormalities extended wider than structural alterations. In patients with ARMD there was generally good correspondence between the area of structural alterations and macular dysfunction; however, most patients did not show significant functional improvements post-treatment even though OCT confirmed improvements in macular structure. In patients with a macular hole, pre-treatment MMI showed varying degrees of functional loss. Post-treatment MMI showed anatomical closure in all patients accompanied by improvements in micro-mfERG amplitudes but persistence of timing delays. The patients tested with other conditions generally showed good correlation between the area of structural alterations and macular dysfunction.

\section{Conclusions}

MMI provides a means of simultaneously imaging the surface, substructure and function of the macula at multiple discrete sites. The results presented illustrate that disease processes do not necessarily result in corresponding deficits in macular structure and function. MMI is well tolerated by patients and may help in improving our understanding of disease processes and in assessing the success of surgical or pharmacological interventions for various disorders affecting the macula.

\section{Oral Session 9: Hereditary retinal disease}

\section{Chairs: Alma Patrizia Torme \& Enid Chelva OS9-Oral Paper 1}

A novel approach analyzing multifocal ERGs: spatiotemporal partial least squares (ST-PLS)

Thomas Wright $^{1,2}$, Filomeno Cortese ${ }^{1}$, Carol Westall ${ }^{1,3}$

${ }^{1}$ Department of Ophthalmology and Vision Sciences, The Hospital for Sick Children, Toronto, CANADA; ${ }^{2}$ Department of Clinical Neurophysiology, Sahlgrenska Academy, Göteborg University, Sweden; ${ }^{3}$ Department of Ophthalmology and Vision Sciences, University of Toronto, Canada

\section{Purpose}

To determine if a multivariate statistical technique, Spatiotemporal Partial Least Squares (ST-PLS), can be used effectively to analyze multifocal ERGs.

\section{Methods}

Typical multifocal ERG analyses have focused on identification of reliable signal changes at the level of individual channels or time-points. A multivariate statistical technique, spatiotemporal Partial Least Squares (ST-PLS) commonly used in neuro-imaging, focuses on the examination of the distributed pattern of all channels and all time-points.

Multifocal ERGs were collected from 12 adolescents (mean 14.7 yrs; min 12; max 21) diagnosed with Type 1 Diabetes for at least 5 years. All subjects underwent retinal examination and those with greater than mild retinopathy excluded. In addition mfERGs were recorded from 13 age-similar (mean $15.6 \mathrm{yrs}$ ) visually normal control subjects. Two mfERG paradigms were recorded from each subject using the Veris ${ }^{\mathrm{TM}}$ system, a standard 103 hexagon recording $(\mathrm{m}=15)$ to examine the outer retina layers and a second, slow flash paradigm where each $\mathrm{m}$-sequence frame is followed by 5 dark frames $(\mathrm{m}=12)$ to isolate the mfOPs.

ST-PLS operates on the entire data structure at once, which requires that the data be in matrix form. Every row of the matrix contains data for one subject. These rows are arranged such that subjects are nested within population group blocks. With $\mathrm{n}$ subjects and $\mathrm{k}$ groups, there are $\mathrm{n} * \mathrm{k}$ rows in the matrix. The columns of the data matrix contain the signal measured for each channel at each time-point. With $\mathrm{m}$ sensors and $\mathrm{t}$ time-points, there are $\mathrm{m} * \mathrm{t}$ columns in the matrix. STPLS computes a matrix containing the covariance of each timepoint for each sensor between two population groups and then analyzes that covariance matrix with Singular Value Decomposition (SVD) to identify a new set of covariance values that correspond to the strongest effects in the data. The resultant patterns identify similarities and differences in the recorded waveforms between population groups.

\section{Results}

No significant pattern of change was observed in the standard mfERG recordings $(P=0.825)$; however, the slow flash mfERG recordings showed differences between the two groups $(P<0.001)$. These differences presented as amplitude decreases and timing delays in a para-foveal ring affecting mfERG rings 2, 3, and 4 .

\section{Conclusions}

The slow flash mfERG paradigm reveals inner retinal changes in subjects with Type 1 Diabetes before vascular changes become obvious under retinal examination. ST-PLS proves to be a robust method for extracting distributed signal changes related to population differences. Univariate methods are optimal for identification of signal changes at specific points in time or space, while multivariate methods such as ST-PLS enable inferences about differences across space and/or time by combining information across these dimensions.

\section{OS9-Oral Paper 2}

On the differentiation between fundus albipunctatus and retinitis punctata albescens

G. E. Holder ${ }^{1,2}$, E. Sohn ${ }^{1}$, L. Zheng ${ }^{2}$, V. M. McBain ${ }^{1}$, A. G. Robson ${ }^{1,2}$, A. R. Webster ${ }^{1,2}$

${ }^{1}$ Moorfields Eye Hospital and ${ }^{2}$ Institute of Ophthalmology, London, UK

\section{Purpose}

To compare and contrast the phenotypic variation in patients with fundus albipunctatus (FA) and retinitis punctata albescens (RPA).

\section{Methods}

A cohort of patients with fundus albipunctatus was assessed by clinical examination, electrophysiology and autofluorescence imaging. Electrophysiology was performed to incorporate the relevant ISCEV Standards; significant additions included the use of red stimulation under dark adaptation and the use of extended dark adaptation (DA; usually one eye overnight). Most patients gave permission for DNA screening which confirmed RDH5 mutation. A cohort of patients with RPA consequent upon CRALBP mutation was similarly ascertained. 


\section{Results}

Patients with FA showed a variable degree of cone dysfunction, with only some patients showing full-field cone ERG abnormalities. Patients with RPA had marked cone system abnormalities. Rod specific ERGs were undetectable in FA patients after standard DA, but there was pronounced recovery of rod system function following extended DA, when rod system ERGs could return to normal. Red stimulation under DA suggested that recordings under DA were arising from the cone system, in keeping with the loss of rod function consequent upon defective regeneration of rhodopsin. Patients with RPA could show limited recovery following extended DA, but normalization was not observed.

\section{Conclusion}

The value of accurate electrophysiological characterization in the differentiation between FA and RPA is confirmed. The importance of an extended period of dark adaptation prior to ERG is stressed.

\section{OS9-Oral Paper 3}

\section{Progression of retinal dystrophy in mucolipidosis IV-an electroretinographic study}

Rafael C. Caruso ${ }^{1}$, Raphael Schiffmann ${ }^{2}$, Janine A. Smith ${ }^{1}$, Ehud Goldin ${ }^{3}$

National Eye Institute, National Institutes of Health, Bethesda, MD (NIH); ${ }^{2}$ National Institute on Neurological Disorders and Stroke, NIH; ${ }^{3}$ National Human Genome Research Institute, NIH

\section{Purpose}

The objective of this study was to describe the rate of decline of retinal function in patients with mucolipidosis IV (ML-IV). This autosomal recessive neurodegenerative lysosomal storage disease is caused by mutations in the MCOLN1 gene, which encodes the protein mucolipin-1, a cation channel. Features of ML-IV include psychomotor retardation, retinal degeneration, corneal clouding, and gastric achlorhydria. We also explored the association between the type of MCOLN1 gene mutation and the ERG phenotype.

\section{Methods}

Seventeen patients (14 females, 3 males), 1 to 24 years old, were included in this study. To study the rate of progression in individual patients, follow-up recordings (from 2 to 4) were obtained in eight patients. Ganzfeld ERGs were recorded following ISCEV standard procedures under general anesthesia with propofol and nitrous oxide. A scotopic intensity-response function was also recorded. The rate of decline of retinal function was estimated using the time constant of an exponential decay model. MCOLN1 gene mutations were identified by sequencing cDNA and genomic DNA.

\section{Results}

All patients, except the youngest, showed reduced b-wave amplitude in standard combined responses. In contrast, a-wave amplitude in standard combined responses was normal for the first 4 years of life. With one exception, all patients 12 years old or older had non-recordable ("extinguished") combined responses. The exception was a 14-year old male with a mild phenotype and no psychomotor retardation (Goldin E et al.,
IOVS, 2008, in press). In the 11 patients under age 12, there was an exponential decline in ERG amplitude with age. The time constant for this decline was 2.63 years for a-waves and 2.65 years for b-waves in combined responses. Other ERG responses showed a similar behavior. Four of the eight patients with follow-up recordings showed a rapid decline of ERG amplitude with time (mean time constant: 2.82 years). The remaining four had a considerably more stable course (mean time constant: 39.11 years). The latter patients all had missense mutations in the MCOLN1 gene.

\section{Conclusions}

This is the largest series of ERG findings in ML-IV patients reported to date. Our results indicate that ML-IV as a rule caused a rapidly progressive retinal degenerative disease. Retinal function was abnormal by 2 years of age, and usually declined to an extremely severe degree by age 12 . Responses generated by bipolar cells were compromised earlier in life than responses generated by photoreceptors. Missense mutations in the MCOLN1 gene caused a less severe and less progressive ERG phenotype than large deletions or splice site mutations. Potential therapeutic interventions designed to treat retinal dystrophy in ML-IV should be instituted very early in life to rescue retinal function.

\section{OS9-Oral Paper 4}

\section{Negative ERG in a patient with subacute sclerosing panencephalitis}

Hiroko Yamazaki ${ }^{1,3}$, Chai Yuzhu ${ }^{1}$, Yutaka Nonoda ${ }^{2}$, Asako Arai $^{2}$, Eiji Nakagawa ${ }^{2}$, Makiko Kaga ${ }^{3}$

${ }^{1}$ Department of Ophthalmology, Kohnodai Hospital, National Center of Neurology and Psychiatry; ${ }^{2}$ Division of Child Neurology, National Center Hospital for Mental, Nervous and Muscular Disorders, National Center of Neurology and Psychiatry; ${ }^{3}$ Department of Developmental Disorders, National Institute of Mental Health, National Center of Neurology and Psychiatry

Subacute sclerosing panencephalitis (SSPE) is a slow virus infection caused by persistent defective measles virus. Ocular manifestations, including cortical blindness, papilledema, optic atrophy and chorioretinitis, were reported in 10-50\% of SSPE patients. The viral inclusions have been demonstrated in the retina and the optic nerve in SSPE.

\section{Purpose}

To explore clinical and electrophysiological findings in a patient with SSPE.

\section{Methods}

The patient was an eight-year-old boy who experienced intellectual deterioration, and behavioral changes began 3 month ago. He was diagnosed as having SSPE because of elevated serum and CSF levels of measles antibodies. Ocular examinations including fluorescein angiography, ISCEV standard ERGs and flash VEPs were performed.

\section{Results}

Visual acuity was 0.6 and 0.6 in his right and left eye, respectively. Light reflex was prompt and full. Intracranial pressure was not elevated. Swollen hyperemic discs with dilated retinal veins were observed. Macula was normal in each 
eye. Fluorescein angiography revealed dilatation of disc capillaries. Flash VEPs were flat. Negative ERGs were recorded to dark adapted bright white flash stimuli in both eyes. Conclusion

Negative ERGs recorded in our patient were a new and an important finding because it suggests that the selective functional impairment of the inner nuclear layer associated with SSPE. Thus we could find selective functional abnormalities explained by the previous pathological reports about ocular lesions of SSPE.

\section{OS9-Oral Paper 5}

\section{CSNB caused by a novel nonsense mutation in CABP4 is characterized by severe cone dysfunction, but normal scotopic vision and normal primary rod pathway}

Maria van Genderen ${ }^{1}$, Karin Littink ${ }^{2}$, Frans Riemslag ${ }^{1}$, Frans Cremers ${ }^{2}$, Ingeborgh van den Born ${ }^{3}$

${ }^{1}$ Bartiméus Institute for the Visually Impaired, Zeist, The Netherlands; ${ }^{2}$ Department of Human Genetics, Radboud University Nijmegen Medical Centre, Nijmegen, The Netherlands; ${ }^{3}$ Rotterdam Eye Hospital, Rotterdam, The Netherlands

\section{Purpose}

Mutations in CABP4 have recently been identified as cause of autosomal recessive congenital stationary night blindness ( $\operatorname{arCSNB}$ ), with a phenotype similar to that of CSNB2. CABP4 is specifically located in the photoreceptor synaptic terminals of both rods and cones. It colocalizes with and modulates the Cav1.4 channel that is encoded by the CACNA1F gene. The purpose of this study was to investigate the psychophysical and electrophysiological features in two patients with a novel CABP4 mutation.

\section{Methods}

In a brother and sister, suspected of cone dystrophy, a novel nonsense mutation in CABP4 was found. Subsequently, they were referred for ophthalmologic and electrophysiological examination. Electrophysiological assessment included an extension of the standard ISCEV protocol, and a "Miyake" protocol. $15 \mathrm{~Hz}$ scotopic measurements were performed to assess the function of the primary and secondary rod pathway. The primary rod pathway, which is the rod ON bipolaramacrine II cell pathway, carries signals with the lowest thresholds, whereas the secondary pathway, which uses rodcone gap junctions, is less sensitive by $\pm 1 \log$ unit. In the normal observer, destructive interference between the signals of both pathways at intermediate intensities results in a flicker null accompanied by a $180^{\circ}$ phase shift.

\section{Results}

The siblings had decreased visual acuity, nystagmus, photophobia, and hypermetropia. Color vision tests were severely abnormal. Dark adapted thresholds were elevated only 0.5 and $0.75 \mathrm{log}$ units in the brother and sister, respectively. The ERG showed normal scotopic responses in the boy, and subnormal responses in the girl, with electronegative mixed responses in both. Photopic ERG responses were severely reduced, with the $30 \mathrm{~Hz}$ responses showing the double peak waveform characteristic of CSNB type $2.15 \mathrm{~Hz}$ scotopic measurements showed intact primary rod pathway responses, but no minimum ERG response or $180^{\circ}$ phase shift, indicating absent or severely subnormal secondary rod pathway responses.

\section{Discussion}

The novel nonsense mutation in CABP4 in the siblings resulted in severe cone dysfunction, including abnormal color vision and photophobia. In contrast, dark adapted thresholds were only marginally elevated and the children did not experience any problems in night vision. Electrophysiological features were remarkable for the normal scotopic ERG in the boy, and normal primary rod pathway responses in both children. The secondary pathway, i.e. the rod-cone gap junction pathway, appeared to be absent. The condition therefore appears clinically as well as electrophysiologically to be dominated by cone dysfunction. Although the mixed and photopic ERGs show similarities to the ERG in CSNB type 2, the term night blindness for this disorder seems inappropriate.

\section{OS9-Oral Paper 6}

The sensitivity and specificity of ISCEV standard ERGs and EOGs when discriminating normal, Retinitis Pigmentosa, and mixed disease

Vaegan $^{1,2}$, Stephanie Yeo ${ }^{1}$

${ }^{1}$ Optometry and Vision Science, University of New South Wales, Australia; ${ }^{2}$ VisionTest Australia, Macquarie St., Sydney, NSW Australia

\section{Purpose}

Vaegan had reported that the fast oscillation (FO) of the EOG seemed to be more specific to Retinitis Pigmentosa than the Arden Ratio, because although RP reduced it less frequently ( $72 \%$ vs. $86 \%$ ), it was seldom reduced in other diseases. We therefore used ROC analysis to compare the sensitivity and specificity of every ISCEV standard EOG and ERG measure for separating normal, Retinitis Pigmentosa, and other diseases. Methods

The data from all normal $(N=58), \mathrm{RP}(N=125)$, and other disease patients $(N=476)$ tested in our clinic over a 13-year period, who had both an ERG and an EOG, were extracted. The same electrophysiologist, diagnosing clinician, and purpose built, regularly calibrated, electrophysiological equipment complying with ISCEV standard conditions were used throughout. Recording programs incorporated fully programmable amplifiers, exponential averaging, and artifact rejection. Six FO cycles preceded the EOG. At least 2 min of reversed change defined the dark trough and light peak. Subpalpebral DTL electrodes were used for ERGs. ERGs terminated when the trace became stable. Photopic ERGs were recorded before dark adaptation (>40 min). ERGs, excluding OPs, were measured using onscreen cursors by an independent assistant and EOGs by the tester. For each measure, the statistics from three empirical ROC curves were calculated to show how they discriminated the groups.

\section{Results}

Five ERG parameters: pure rod amplitude, bright scotopic b amplitude, bright scotopic a amplitude, photopic b amplitude, and flicker amplitude all discriminated RP from normal with an area under the ROC curve (AUC) of $>0.948$. The first two of 
these also discriminated RP from other diseases with an AUC of $>0.90$ and the next two with an AUC (>0.84). Both photopic amplitudes discriminated normal from other diseases with an AUC $>0.725$. Arden Ratio and Light Peak discriminated RP from normal with an AUC $>0.9$ which surpassed the FO (0.86). The Arden Ratio was also best at discriminating normal from mixed (0.72) and RP from mixed (0.80). The light peak amplitude (0.78) and FO (0.77) discriminated RP from other diseases similarly.

\section{Conclusions}

There were always several ERG measures with larger AUC than any EOG score. The Arden Ratio was always the best EOG measure and the light peak next best. The similarity of the FO to the Arden Ratio, especially in separating RP from other diseases, partly supports Vaegan's initial observation. The analysis also provided d prime values for the $50 \%$ separation points between groups. These could be quite different to the normal limit and hence useful for differential diagnosis.

\section{Support}

An untied grant from Allergan (Vaegan) and a Summer 2007/ 2008 research scholarship from the Faculty of Science, University of NSW (Stephanie Yeo).

Acknowledgements Paul Beaumont made all initial diagnoses. Dr Jennifer Sandbach grouped the initial diagnoses into a smaller set of subcategories for the first analysis. George Ivanov and Brian Lowrey measured the ERGs.

\section{OS9-Paper 7}

Principal component analysis of multifocal electroretinogram in Retinitis Pigmentosa

Aniruddha Maiti, Mahesh Uparkar, Sundaram Natarajan

Aditya Jyot Eye Hospital Pvt Ltd., Wadala,

Mumbai-31, India

\section{Purpose}

To determine waveforms of mfERG in Retinitis Pigmentosa (RP) those contribute significantly to the overall retinal response.

\section{Methods}

The subjects were 25 consecutive patients with bilateral typical Retinitis Pigmentosa seen at a single tertiary referral ophthalmic center in Mumbai city from January 2005 to January 2007 for a prospective consecutive non-randomized observational casecontrolled study from a single tertiary ophthalmic center over two years. A detailed history was sought on all individuals for familial affection and concomitant systemic disease. Bestcorrected distance VA was assessed on a logMAR (logarithm of the minimum angle of resolution) scale by using the backlit Early Treatment Diabetic Retinopathy Study charts (ETDRS). Slit-Lamp biomicroscopy, applanation tonometry, and binocular indirect ophthalmoscopic examination were done in each subject. Multifocal ERG testing stimulation and primary analysis were performed using a stimulus-camera-refractor unit (VERIS, ver. 5.1.12 X; Electrodiagnostic Imaging [EDI] San Mateo, CA).

Results

Male preponderance was seen in RP cases (22, OR: $13.68,95 \%$ CI: $3.85-49.88, P=0.000)$. Principal component analysis using varimax rotation for the latencies and amplitudes in RP revealed two components (from central 10 degrees of macula) each contributing to $66.8 \%$ and $88.8 \%$ of the total variance, respectively.

\section{Conclusion}

MfERG ring1 and 2 responses from the central 10 degrees contribute to the maximum variance of waveforms in RP irrespective of visual acuity.

\section{Poster Session C: Electrophysiological testing-EOG, mfERG, VEP methods}

\section{Chairs: Subhatra Jalai \& Scott Brodie}

\section{Poster C1}

\section{Hypotension and electro-oculogram}

Yuzo Miyake, Atsuhiro Tanikawa, Mitsuo Sugimoto, Masayuki Horiguchi

Department of Ophthalmology, Fujita Health University, Toyoake, Japan

\section{Purpose}

The amplitude of EOGs is reduced in eyes with retinal detachment (RD) accompanied by choroidal detachment (CD), but it is normal in eyes with RD not accompanied by $\mathrm{CD}$ (Miyake et al., Retina, 1993). EOG may provide useful information on the status of choroid in various conditions, especially when the ultrasound biomicroscopy (UBM) cannot be used. In the present study, we analyzed the intraocular pressure (IOP), EOG, and UBM findings in several diseases in order to investigate the mechanisms of reduction in the EOG amplitude.

\section{Methods}

The IOP measurement, EOG recording, and UBM were performed in traumatic hypotony maculopathy, fresh uveal effusion syndrome (UE), and longstanding hypotony after uncomplicated trabeculectomy. The EOG amplitude in the affected eye was compared to that of the normal fellow eye.

\section{Results}

The table below showed the results of IOP, UBM findings, and EOG amplitude in each disease. Reduction of EOG amplitude was observed only when both CD and low IOP existed. In hypotony maculopathy, aqueous humor leaks into subchoroidal space through the angle break caused by trauma, and in RD with $C D$, vitreous fluid leaks into subchoroidal space through the retinal break and retinal pigment epithelium. The shift of the intraocular fluid into subconjunctival space did not reduce the EOG amplitude in hypotony after trabeculectomy, and accumulation of subchoroidal fluid from choroidal vessels did not decrease the amplitude in UE.

\section{Conclusions}

These results suggest that pathological flow of the intraocular fluid (aqueous humor or vitreous fluid) into subchoroidal space reduces the amplitude. In early postoperative period of intraocular surgery, hypotony may be associated with $\mathrm{CD}$ that induces vision-threatening complications; however UBM cannot be used. The amplitude of EOG may be useful for early detection of $\mathrm{CD}$ after intraocular surgeries. 


\section{Poster C2}

\section{Bilateral maculopathy with subretinal neovascularization in a patient with Charcot-Marie-Tooth disease}

Richard MacMaster Francis, Jr., Lionel Chisholm, Monique Leys

West Virginia University Eye Institute, Morgantown, WV 26506, USA

\section{Purpose}

Charcot-Marie-Tooth disease is the most common inherited disorder of the peripheral nervous system. The disease is caused by mutations in several different myelin genes, which result in defects in myelin structure, maintenance, and formation. Motor and sensory nerves throughout the peripheral nervous system are affected. Cases associated with ophthalmic abnormalities and visual impairment are rare. We examined one patient with visual loss and diplopia and reviewed the literature.

\section{Methods}

We examined a 29-year-old WF with Charcot-Marie-Tooth disease and a bilateral maculopathy resembling Best disease. She had a 5 years smoking history and also suffered from narcolepsy. Visual acuity was 20/40 in both eyes. The right eye had subretinal fibrosis in the macula suggestive of previous subretinal neovascularization. In the left eye, she had a macular hemorrhage associated with classic subretinal neovascularization.

\section{Results}

The patient received 2 intravitreal bevacizumab injections. Subsequently, visual acuity improved from 20/40 to 20/25 and has been stable for 14 months. EOG light rise was normal: LP/ DT ratios were $2.89 \mathrm{OD}$ and $2.30 \mathrm{OS}$. The patient's medical record, including neurologic evaluation and nerve conduction study results, was reviewed.

\section{Conclusions}

The association of inherited neuropathy and macular dystrophy with subretinal neovascularization is rare. This is the first report, to our knowledge, of its successful treatment with intravitreal bevacizumab.

\section{Poster C3}

\section{Retinal function in diabetic retinopathy with macular edema}

Mariam Arakelian ${ }^{1}$, Natalia Mansurina ${ }^{1}$, Yulia Bardeeva ${ }^{2}$, Angelika Shamshinova ${ }^{1}$

${ }^{1}$ Moscow Helmholtz Institute of Eye Diseases, Moscow, Russia; ${ }^{2}$ Moscow Regional Research Clinical Institute, Moscow, Russia

\section{Purpose}

To investigate the functional changes of macular area in type 2 of diabetes mellitus depending on the clinical presentation of retinal changes.

\section{Methods}

25 patients (37 eyes) with diabetic retinopathy (DR) were included in our study: nonproliferative DR (NDR)-14 eyes, preproliferative DR (PPDR)-12 eyes, proliferative DR
(PDR)—11 eyes. All patients had clinically significant macular edema (CSME). ISCEV Standard tests as well as chromatic macular ERG to red, green, and blue stimuli (MBN, Russia) and RETIScan system (Roland Consult, Germany) for multifocal ERG (mfERG) were used.

Results

Patients with NPDR had normal values of the full-field and flicker ERG. No significant changes of full-field and flicker ERG were revealed in PPDR patients except 4 eyes with subnormal amplitude values. In most patients with PDR (9 eyes), the full-field and flicker ERG showed subnormal values and a delay of b-wave latency. A decrease of OPs with pathological configuration and interpeak latency delay were in accordance with the severity of fundus changes as an evidence of inner retinal layer ischemia. Macular ERGs to red, green, and blue stimuli were subnormal (up to $50 \%$ comparing with normal data) in all three groups of patients. Also mfERG showed considerable relative amplitude decrease and implicit time delay in the central area of $15-22^{\circ}$ of visual angle. Thus, the presence of CSME in all patients determined significant changes of macular ERGs and mfERG with slight difference between groups, that is the deterioration of macular function was not well correlated with the severity of diabetic retinopathy of the whole retina (except its central part).

\section{Conclusions}

Electrophysiological symptoms of macular area in different stages of DR with CSME in type 2 diabetes mellitus must be considered as a severe complication and one of the most predominant causes of vision loss, especially in patients with NPDR and PPDR, which require early detection of macular edema for urgent treatment and better visual outcome.

\section{Poster C4}

Characteristics of global flash multifocal electroretinogram (mfERG) under different combinations of global and focal flash luminance

Jenny C. Y. Lung, Patrick H. W. Chu, Y. F. Ng, Henry H. L. Chan

Laboratory of Experimental Optometry (Neuroscience), School of Optometry, The Hong Kong Polytechnic University, Hung Hom, Kowloon, Hong Kong SAR, China

\section{Purpose}

This pilot study is to investigate the characteristics of the response triggered by the global flash mfERG under various combinations of global and focal flash luminance and to figure out the optimal conditions for this measurement.

\section{Methods}

Seven normal subjects with mean age $24.3 \pm 2.21$ years were recruited to undergo the measurement of the global flash mfERG consisted of four video frames (103 scaled hexagonal $\mathrm{m}$-frame, followed by a dark frame, a global flash and then another dark frame). The focal and global flash intensities were varied independently for four levels (from 0.63 to $5.54 \mathrm{~cd} / \mathrm{m}^{2}$ / s). It was then resulted in 16 combinations of focal and global flash luminance. The direct component (DC) and induced component (IC) were grouped into central and peripheral region for further analysis. The proportion of the global flash 
and focal flash intensity was studied so as to achieve the optimal IC response.

\section{Results}

Under the conditions with the focal flash intensity (f) brighter than the global flash intensity (g) (i.e. g < f), both central and peripheral DC amplitudes were greater than those measured under the conditions with the global flash brighter than the focal flash (i.e. $g>$ f). Under the conditions with the global flash intensity weaker than the focal flash intensity (i.e. $g<f$ ), the IC amplitude increased and achieved to the peak value when the global flash intensity was double of the focal flash intensity (i.e. $g=2 \mathrm{f}$ ). Beyond this ratio, the IC value dropped. When the global and focal flash intensities were $g=2 f$, the performance of the IC was different between central and peripheral regions. The central IC amplitude dropped with increasing focal flash intensity, while the peripheral IC amplitude increased with focal flash intensity (from 0.63 to $2.75 \mathrm{~cd} \mathrm{~s} / \mathrm{m}^{2}$ ).

\section{Conclusion}

The direct component (DC) and induced component (IC) in the global flash mfERG are suggested from the outer and inner retinal activity, respectively. Different combinations of these two flash intensities would provide different preference in measuring the DC and IC from different retinal regions. For the investigation of the peripheral inner retinal response, a moderate focal flash intensity $\left(1.43-2.75 \mathrm{~cd} / \mathrm{m}^{2} / \mathrm{s}\right)$ with $\mathrm{g}: \mathrm{f}=2: 1$ is recommended to use in order to achieve considerable DC and IC amplitudes. For the investigation of the central inner retinal response, a relatively weak focal flash intensity $\left(0.63 \mathrm{~cd} \mathrm{~s} / \mathrm{m}^{2}\right)$ is enough to trigger considerable DC and IC amplitudes.

\section{Support}

Competitive Earmark Research Grants (PolyU 5384/04 M and PolyU 5415/06 M) from The Research Grants Committee of The Hong Kong SAR, the Niche Areas-Glaucoma Research (1-BB76) and the Associated Fund (Research Postgraduate) from The Hong Kong Polytechnic University.

\section{Poster C5}

\section{The techniques and system for multifocal electroretinography}

Aram Arakelyan

Department of Information Technology, University of International Economic Relations, Yerevan, 0025, Armenia

\section{Purpose}

To develop the techniques and system for multifocal electroretinography targeted for the investigation of the retina responses to color as well as white and black stimuli. The approach takes into account the density of the allocation of rods and cones in retina.

\section{Methods}

The testing method is based on the following steps. As the first step the value of the radius of circle formed by hexagonal segments is set up. Then values of width of rings sequence formed by hexagonal segments are set up. The stimulus generation according to binary a-sequences (BAS) is implemented. The dialogue screen allows a selection of the frequency of retina stimulation, number of hexagonal segments, pairs of stimuli colors (flash and background), frequency of mfERG recording. Because cones and rods have different sensitivities to stimuli colors, the new method allows generation of continuous (color background and color flash, black background and white flash) stimulation sequences selectively depending on the density of allocation of rods and cones in the retina.

Results

The stimulator we developed allows stimulation of retina depending on the density of allocation of rods and cones in retina. Listed features substantiate the fact that the new method to generate stimuli is unique and is different from the other methods and could be used as the basis for a new technology in clinical electrophysiology and neurophysiology of vision. The software provides selection of defined number of hexagons in each area, an implementation of flashes of specified frequency and duration according to the automatically selected options. Algorithms of the stimuli generation are based on binary asequences (BAS). The diversity of BAS allows making a choice between existing different classes and so recording retina responses to these stimuli. Consequently, it is also possible to receive multifocal images as responses to stimuli. Conclusions

Developed techniques of mfERG is a promotion of existing methods and could be used in clinical practice.

\section{Poster C6}

Electrophysiological and morphological changes after intravitreal bevacizumab injection in patients with macular edema and choroidal neovascularization

Young-Hoon Ohn ${ }^{1}$, Soo Young Lee ${ }^{2}$, Hyun Joon Lee ${ }^{1}$

${ }^{1}$ Department of Ophthalmology, Soonchunhyang University Hospital, Bucheon, South Korea; ${ }^{2}$ Department of Ophthalmology, Ewha Womans University Mokdong Hospital, Seoul, South Korea

\section{Purpose}

To investigate the functional and morphological changes after intravitreal bevacizumab (Avastin ${ }^{\circledR}$ ) injection in patients with macular edema and choroidal neovascularization(CNV) using mfERG and Optical Coherence Tomography (OCT).

Methods

Intravitreal bevacizumab $(0.075 \mathrm{ml})$ injection was performed one to three times on twenty-one eyes of 21 patients diagnosed as diabetic macular edema, age-related macular degeneration (ARMD), and branch retinal vein occlusion (BRVO). mfERG and OCT were recorded before and after the injection to analyze the macular function and central retinal thickness (CRT).

\section{Results}

Mean CRT decreased from 283.1 to $235.2 \mu \mathrm{m}$ at 3 months after the injection on OCT. Mean $\mathrm{P}_{1}$ and $\mathrm{N}_{2}$ latency of ring 1 were decreased, and $\mathrm{N}_{1}$ and $\mathrm{P}_{1}$ amplitudes of ring 1 and $\mathrm{N}_{1}$ amplitude of ring 2 were increased at 3 months after the injection on mfERG. The most sensitive parameter was $\mathrm{P}_{1}$ latency of ring 1 .

\section{Conclusions}

These results suggest that mfERG and OCT were useful to assess the effect of intravitreal bevacizumab injection in 
patients with macular edema and CNV. However further study is needed to confirm the long-term effect of the treatment because only a few parameters were statistically significant.

\section{Poster C7}

\section{Multifocal electroretinogram progression in patients with RP}

Karen Holopigian $^{1}$, Jenny M. Gallardo ${ }^{1}$, William Seiple ${ }^{1}$, William H. Swanson ${ }^{2}$, Ronald E. Carr ${ }^{1}$

${ }^{1}$ New York University School of Medicine, New York, NY;

${ }^{2}$ Indiana University School of Optometry, Bloomington, IN

\section{Purpose}

The mfERG has become a standard tool for documenting conemediated retinal function in patients with Retinitis Pigmentosa (RP). The mfERG may be useful for measuring disease progression, because many patients with RP have measurable responses within the area tested with a standard mfERG paradigm. However, little is known about the rate of progression in patients with RP, especially as a function of retinal eccentricity. In current study, we examined changes in the amplitude and implicit times of the mfERG over time and retinal eccentricity in patients with RP.

\section{Methods}

Monocular mfERGs were obtained on twenty-six patients with RP over follow-up periods ranging from two to nine years. Patients included had visual acuities of $20 / 40$ or better and Goldmann visual fields (V4e) of $>10$ degrees at their initial visit and recordable mfERGs on two or more visits. The mfERG recordings were done with a VERIS system and a bipolar Burian-Allen electrode. The stimulus was a scaled array of 103 hexagons subtending 46 degrees (mean luminance $=100 \mathrm{~cd} / \mathrm{m}^{2}$ ). Each of the 103 hexagons was evaluated independently and values of relative amplitude and implicit time (a-scale and t-scale) were obtained using a Matlab analysis program (Hood and Li, 1997).

\section{Results}

The results were analyzed separately for three rings: ring 1 (the fovea and surrounding hexagons, to $5^{\circ}$ ), ring 2 (to $10^{\circ}$ ), and the periphery (all remaining points to $23^{\circ}$ ). The data were averaged in linear units and $\log$ transforms were performed for analysis. For each patient and each ring, a-scale and t-scale were plotted as a function of years of follow-up and linear regression was used to derive the rate of decline in $\mathrm{dB} /$ year. Analysis of variance was used to determine if there were significant differences for the three rings. For amplitude, the median loss at the initial visit (intercept) was $6.05 \mathrm{~dB}$ for ring $1,8.76 \mathrm{~dB}$ for ring 2, and $10.45 \mathrm{~dB}$ for ring 3 . The median slope (the rate of decline) was -0.001 for ring $1,-0.26$ for ring 2 , and -0.31 for ring 3 . For implicit time, the loss at the initial visit was $0.21 \mathrm{~dB}$ for ring 1 , $0.75 \mathrm{~dB}$ for ring 2 , and $01.27 \mathrm{~dB}$ for ring 3 . The median slope (increase in implicit time) was -0.004 for ring $1,0.015$ for ring 2 , and 0.101 ring 3 . For both amplitude and implicit time, the initial loss was significantly different for ring 1 vs. ring 3 . There were no significant differences in slope for either measure.

\section{Conclusions}

We found that mfERG amplitude and implicit times show progression over the follow-up period. There were no differences in the rate of progression with eccentricity for either measure. This result could indicate linear losses in photoreceptor function with eccentricity.

Support

Foundation Fighting Blindness.

\section{Poster C8}

\section{Foveal dystrophy or dysfunction: a clinical entity}

N. Khan, J. R. Heckenlively

University of Michigan, Kellogg Eye Center, Ann Arbor, MI

\section{Purpose}

To demonstrate that the mfERG is an effective tool for the detection of foveal dysfunction in the face of a normal standardized ERG and other functional testing in patients complaining of subnormal vision and who have no foveal changes.

\section{Methods}

Eight patients aged 16-62 years with complaints of reduced acuity were assessed with visual acuity, color vision testing (CV), Goldmann visual fields (GVF), mfERG (multifocal ERG), full-field ERG, fundus ophthalmoscopy, fundus photos, and optical coherence tomography (OCT). The mfERG was recorded as a scaled array of 103 hexagons and the amplitude and implicit time of six concentric rings were compared to those of normal control subjects $(n=15)$. For each ring, the peak amplitude (difference between P1 and N1) and the implicit time of P1 were compared with controls.

\section{Results}

In all subjects, acuity ranged from 20/30 to 20/400 in the worse eye. Color vision, tested with Ishihara Plates and the Farnsworth D-15, ranged from normal to severely affected color discrimination. GVFs were normal in six subjects and two subjects had central scotomas. Dark-adapted and light-adapted ERGs were normal in all subjects. Fundus examinations revealed no overt degeneration in the macular region. OCTs, which were done on 6 patients, showed broadening and thinning of foveal pits. mfERGs showed significant amplitude reduction in the central rings 1 and 2 when compared to control.

\section{Conclusions}

Significant reduction in mfERG with normal Ganzfeld ERG was found in a group of patients who otherwise might have been thought to be malingering. Localized loss of function may indicate loss of cone photoreceptors or damage to the cone photoreceptor outer segments. The mfERG is clinically useful in evaluating foveal functional pathology in the presence of a normal full-field ERG.

\section{Support}

Foundation Fighting Blindness. 


\section{Poster C9}

\section{Correlation between vertical kinetic visual acuity and visual evoked potential in patients with unilateral optic neuritis}

\author{
Yujin Mochizuki ${ }^{1}$, Atsushi Mizota ${ }^{1}$, Minoru Tanaka ${ }^{1}$, \\ Yoshimitsu Kohmura ${ }^{1}$, Hiroshi Yoshigi ${ }^{1}$ \\ ${ }^{1}$ Department of Ophthalmology, Juntendo University \\ Urayasu Hospital, Urayasu, Japan; ${ }^{2}$ Doctoral Program in \\ Sports Science, Juntendo University, Shisui, Japan; \\ ${ }^{3}$ Department of Sports Science, Juntendo University School \\ of Health and Sports Science, Shisui, Japan
}

\section{Purpose}

To determine whether a significant correlation exists between the vertical kinetic visual acuity (VKVA) and the peak latency of the pattern reversal VEPs in patients with unilateral optic neuritis.

\section{Subjects and methods}

Eight patients ( 2 men and 6 women, mean age 35.6 years) recovered from acute unilateral optic neuritis with corrected visual acuity $\geq 20 / 20$ were studied. The static visual acuity (SVA), VKVA, and pattern reversal VEPs were recorded. The SVA and VKVA were measured with a kinetic visual acuity meter (AS-4F, KOWA, Tokyo, Japan). VEPs were amplified and the responses were signal averaged (Neuropac system, Nihon Koden, Tokyo, Japan). Black and white checkerboard pattern was used. The overall size of the stimulus was $12^{\circ} \times 15^{\circ}$, and the size of the checks was $60 \mathrm{~min}$ with reversal frequency of 2 reversals/s. The peak latency of the P100 component was measured. Both SVA and VKVA were calculated in $\log$ MAR.

\section{Results}

The peak latency of P100 was statistically significantly delayed in affected eyes $(P<0.001)$. There were no significant differences in SVA between affected eyes and contralateral eyes. VKVA in affected eyes was significantly worse than those of contralateral eyes $(P=0.003)$.

\section{Conclusions}

These findings suggested that P100 component of VEP might have some correlations with the VKVA.

\section{Poster C10}

\section{Blue fixation stimulus to record pattern VEP in optic nerve disease}

Rohit Shetty, Ajoy Vincent, Bhujang Shetty

Department of Electrophysiology, Narayana Nethralaya, Super Specialty Eye Hospital and Post Graduate Institute, Bangalore, India

\section{Purpose}

To report the efficacy of a blue fixation spot in the centre of pattern VEP stimulus monitor in subjects with optic neuritis and optic neuropathy.

\section{Methods}

Prospective, nonrandomized, cross-sectional, case series study was done after institutional ethical committee clearance.
Twenty-two subjects with optic neuritis/optic neuropathy who satisfied the inclusion and exclusion criteria underwent detailed ophthalmological evaluation including best-corrected visual acuity, colour vision, pupil evaluation, fundus evaluation and imaging of the optic nerve. Pattern VEP was done with $60^{\prime}$ and $120^{\prime}$ checker pattern with a red fixation stimulus in accordance to ISCEV guidelines. Pattern VEP was also done to with $60^{\prime}$ and $120^{\prime}$ checker pattern with blue fixation stimulus in the centre of the monitor. Mono-ocular recording using single channel with midline scalp electrodes were done. Eight eyes of eight subjects could not see the red fixation stimulus at the centre of the screen at the testing distance but could see the blue stimulus at the centre. All the rest who could see both the red and the blue fixation stimulus served as control.

Results

The eight eyes of eight subjects who were unable to see the red fixation spot had poorly recordable pattern VEP to that setting. However, their pattern VEP was recordable but abnormal with the blue fixation spot in the centre. The P1 amplitude and P1 latency parameters to both red and blue stimulus fixation spots were comparable in the subjects who could see both types of stimulus fixation spots.

\section{Conclusion}

Visualization of the central fixation spot is essential to elicit waveforms of pattern VEP recording. Optic neuritis and optic neuropathy causes red-green colour vision defects. Hence, a blue fixation spot helps to get a pattern VEP recording in most of these cases. This is helpful in clinical scenario as it may be used for follow-up of cases after appropriate treatment.

\section{Poster C11}

\section{Abnormal pattern visual evoked potential in occult macular dystrophy —is it the nerve?}

Ranjana Mathur ${ }^{1,2}$, Doric Wen Kuang Wong ${ }^{1,2}$

${ }^{1}$ Singapore National Eye Centre; ${ }^{2}$ Singapore Eye Research Institute

\section{Case report}

A 42-year-old healthy male was referred to our electrophysiology laboratory to assess cause for poor central vision. He is the youngest in the family of 8 siblings, with possible similar symptoms in the eldest brother. He noticed difficulty in reading for 2-3 years (OD $>\mathrm{OS}$ ), which was worse at night. Bestcorrected visual acuity was 6/45 OD and 6/30 OS. On ocular examination, there was no evidence of RAPD. His color vision was essentially normal when tested by D-15 Farnsworth color vision test. Ishihara test, although abnormal, did not show particular pattern of color vision deficiency. Slit-lamp examination was within normal limits. Dilated fundus examination showed normal optic discs, absence of nerve fiber defects, parafoveal mild age-related RPE changes sparing the macula further confirmed by Fluorescein angiography. The optical coherence tomography confirmed bilateral macular atrophy (180-190 $\mu$ ). The Humphrey's Visual field showed presence of central scotoma (OD > OS). The Goldmann visual peripheral field was within normal limits. The ISCEV standard full-field ERG was within normal limits. The pattern ERG and pattern VEP (recorded on two different visits) showed normal P50 
amplitude response with reduced/absent N95 with N95:P50 ratio of $1.0 \mathrm{OD}$ and $0.9 \mathrm{OS}$. The pattern VEP responses were severely attenuated for all check sizes on both occasions. The Multifocal ERG showed reduced responses from the central rings $(\mathrm{OD}>\mathrm{OS})$ with no delay or reduction in the outer 3 rings. These findings are typical of occult macular dystrophy (OMD) and led us to diagnose OMD in him. Literature review revealed one case-report of patient with OMD with delayed PVEP responses (Prof. Miyake et al.). In our patient as well, severely reduced PVEP in the absence of other markers of optic neuropathy suggests that PVEP findings are probably due to the macular disease.

\section{Poster C12}

\section{Recording of visual evoked potential in photophobia patients}

\section{Mirdehghan, S. M. Shushtarian, M. S. Mirdehghan}

Tehran Medical Branch, Islamic Azad University, Tehran, Iran

\section{Purpose}

Recording of VEPs is important in diagnosis of demylinating diseases and optic nerve atrophy. It is observed that selection of proper stimulation technique is to be taken into consideration in different pathological conditions. Photophobia is among the conditions that selection of proper stimulation technique is helpful in better diagnosis of this type of disease; therefore VEP using two routine stimulation techniques, i.e., pattern reversal checker board and flash, was tested in photophobia patients to search for possible changes.

\section{Method}

Seventy-five photophobia patients were selected randomly. VEP was tested using pattern reversal checker board and flash stimulations in the total population; VEP P100 was measured. Result

The result shows delay in latency of VEP P100 in case of flash rather than pattern stimulation, The reason for this delay may be the improper response light stimulus by visual system which will be discussed in detail in full paper.

\section{Conclusion}

From the result of present work one can conclude that pattern reversal checker board is a proper stimulation technique for recording VEP in photophobia patients.

\section{Poster C13}

\section{VEPs in response to cone isolating stimuli in college} students with reading discomfort

William H. Ridder III ${ }^{1}$, Christopher Chase ${ }^{2}$, Chinatsu Tosha ${ }^{2}$, Anna Tong ${ }^{1}$, Dani Smith ${ }^{2}$, Eric Borsting ${ }^{1}$

${ }^{1}$ Southern California College of Optometry, Fullerton, California; ${ }^{2}$ Claremont McKenna College, Claremont, California

\section{Purpose}

College students often complain of reading discomfort (also called asthenopia, visual fatigue, visual stress, or the Meares-
Irlen syndrome). Symptoms of reading discomfort include unpleasant somatic and perceptual effects, such as eye-strain, headache, and blurred text, despite normal visual acuity. Several studies have suggested that the symptoms may result from increased neural noise that can be minimized by wearing colored filters. Thus, there may be abnormal neural processing in the cone pathways. A previous investigation of ERG contrast gains indicated that normal and reading discomfort subjects were not significantly different for M-cone isolating stimuli $(P=0.47)$ but approached significance for L-cone isolating stimuli $(P=0.086)$ (Ridder III WH et al (2008) Optom Vision Sci 85: In press). The present study used L- and M-cone isolating stimuli and measured VEPs in normal and reading discomfort subjects to determine if abnormalities in these processing channels become evident in post-retinal processing. Methods

Twelve normal and nine reading discomfort college students were examined. Reading discomfort subjects had Conlon survey scores greater than 1.5 SD above the mean of the population (Conlon et al. 1999). The stimuli (30 Hz flicker) were displayed on a CRT monitor $\left(85^{\circ}(\mathrm{H}) \times 69^{\circ}(\mathrm{V})\right)$. Cone absorptions were estimated from Stockman fundamentals. Silent substitution was used to construct cone-isolating stimuli. Cone-contrast values were computed as the Michelson contrast based on Stockman fundamentals using the predicted coneexcitations of the stimulus relative to the predicted cone excitations of the mean gray background $\left(85 \mathrm{~cd} / \mathrm{m}^{2}\right)$. VEPs were determined monocularly for a range $(1-15 \%)$ of L- and M-cone contrasts. VEP data were acquired and analyzed with a Biopac Lab PRO System. Slopes were determined for the L- and M-cone VEP amplitudes for each subject.

\section{Results}

The average VEP slopes for the reading discomfort group were $0.67 \pm 0.317$ (L-cone) and $0.75 \pm 0.279$ (M-cone). The slopes for the normal subjects were $0.51 \pm 0.317$ (L-cone) and $0.75 \pm 0.327$ (M-cone). There were no significant differences between the normal and reading discomfort groups in the slopes (L-Cone, $P=0.30$; M-Cone, $P=0.98$ ).

\section{Conclusions}

L- and M-cone isolating VEP slopes were not significantly different. These results do not support the noisy visual system hypothesis of reading discomfort.

\section{Poster C14}

\section{Effect of whole body vibration on visual system using} visual evoked potentials

Aryandokht Fotros, S. M. M. Shushtarian

Department of Medical Physics, Tehran Medical Branch Islamic Azad University, Tehran, Iran

\section{Purpose}

Vibration is physically defined as a rapid linear motion of a particle or of an elastic solid about an equilibrium position. Vibrations are more often undesirable and may cause adverse effects on different parts of the body. Nervous system, mainly the auditory one is severely affected by vibrations. Visual cortex is a tiny part of nervous system that is not considered much in this regard; therefore the present work was designed to workout the possible effect of vibration on visual cortex. 


\section{Methods}

To precede the research work a textile factory with a number of machinery creating high levels of vibration was selected. 28 labors with no history of visual impairment were selected. The labors had at least 6 years of experience in the place where the high vibrating machines were operating. VEP was recorded in the case of the labors. Flash amplitude and latency of VEP/ p100 were recorded for each labor. 28 normal subjects were also selected as controls to compare the two groups.

\section{Results}

The mean latency of VEP, P100 peak of the workers exposed to heavy vibration, is $138 \pm 34$ whereas this value is $85 \pm 5$ in normal population.

\section{Conclusion}

The significantly higher $(P<0.005)$ evoked potentials in case group show the effect of vibration on visual system, mainly visual cortex, which reflects itself in latency, amplitude of VEP $\mathrm{P} 100$, and the shape of the waveform which will be discussed.

\section{Poster C15}

\section{Retinal (macular) diseases are easier distinguished from optic neuropathies if electrophysiology is complemented by optical coherence tomography (OCT)}

Hannes Wildberger, Daniel Barthelmes, Oliver Bergamin

Department of Ophthalmology, University Hospital, University of Zurich, Switzerland

\section{Purpose}

To demonstrate structural differences between retinal (macular) disease and optic neuropathies using OCT (Zeiss Stratus). Methods

Four OCT parameters (foveolar depth (FD), foveal area thickness (FA), perifoveal retinal thickness from the inner ring (FR), and axonal thickness of the papillomacular bundle (PMB)) were measured. The PMB is best represented by the temporal $30^{\circ}$ sector of the circumpapillary RNFL. The four parameters were compared in a normal group, in a maculopathy group and in an optic neuropathy group. Electrophysiology (mfERG, VEP and $\mathrm{mfVEP}$ ) was recorded in the affected groups: maculopathies were identified by mfERG signal abnormalities and reduced VEPs; neuropathies were identified by abnormal VEPs alone, while mfERG was normal.

\section{Results}

In maculopathies, FD was reduced in most cases according to the severity of the structural loss. This held also true for the FA and for the FR, although the automated measurement of FA was less precise due to eventual eccentric fixation (FD was measured manually). The most reliable results were obtained from the perifoveal retinal thickness measurement from the inner ring (FR). Thickness loss was less pronounced in mild cases, but more pronounced in severe or advanced stages of macular disease. Structural loss was most present in cones followed by the outer nuclear layer and the Henle fiber layer. A RNFL-thickness loss of the PMB was seldom present, but as an exception, when an obvious severe retinal dystrophy reached the border of the disc.
In optic neuropathies involving the PMB, two features occurred regularly together: a perifoveal retinal thickness loss of the inner ring (FR), probably due to a loss of the ganglion cell layer, and a significant thickness loss of the PMB. Looking at structures always showed intact outer retinal layers but a loss of reflectivity at the inner retinal surface. The FR loss did not exceed a certain value, because ganglion cell loss keeps quantitative limits while structural loss in retinopathies is nearly unlimited in advanced cases. In optic neuropathies, a reduced FD occurred seldom and, if present, to a minor extent only.

\section{Conclusions}

OCT is an important additional diagnostic tool which provides fast and reliable support in different situations of unexplained visual loss. This is not only the case for hereditary conditions but also for acquired problems of various origins.

\section{Poster C16}

\section{Effects of nicotine on the adult electroretinogram}

\author{
S. B. Varghese ${ }^{1}$, E. E. Hartmann ${ }^{2}$, J. C. Reid ${ }^{2}$, K. T. Keyser ${ }^{1}$
}

${ }^{1}$ Vision Science, University of Alabama at Birmingham, Birmingham, AL; ${ }^{2}$ Optometry, University of Alabama at Birmingham, Birmingham, AL

\section{Purpose}

To examine the effects of nicotine on information processing in the human retina by use of the ERG.

\section{Methods}

ERG responses were obtained from eighteen healthy nonsmokers. All individuals were evaluated as visually normal on a comprehensive eye exam and visual field. Subjects were tested in two separate trial sets. Each trial set included one dosage of nicotine gum and a placebo condition. Dosage of nicotine gum for the first trial set was randomly assigned (2 vs. $4 \mathrm{mg}$ ). For the second trial set each subject was tested on the dosage level not previously used. Order of test session (placebo gum versus nicotine gum) was also randomized for each trial set. The experimenter was masked to the type of gum assigned until after each trial set was completed. ERG responses were collected and analyzed using the Espion ERG system under standard ISCEV conditions. Responses were recorded from one eye for each subject using a Burian-Allen lens. Intensity response curves and OPs were obtained under both scotopic $\left(1.41^{-5}-0.921 \mathrm{~cd} / \mathrm{m}^{2}\right)$ and photopic $\left(0.283-35.84 \mathrm{~cd} / \mathrm{m}^{2}\right)$ conditions. The Naka-Rushton equation was used to fit both scotopic and photopic stimulus-response series and paired Student $t$-test was used for statistical analysis. All research reported was conducted in compliance with the Declaration of Helsinki.

\section{Results}

The average subject age was 24.3 years (range $=20-32$ ). Means for $V_{\max }$ and $K$ of the Naka-Rushton function for the photopic series under each condition are shown in the table below. Placebos for both trial sets were averaged together. 


\begin{tabular}{lll}
\hline $\begin{array}{l}\text { Photopic ERG } \\
\text { Condition }\end{array}$ & $\begin{array}{l}V_{\max } \\
\text { Mean (SD) }\end{array}$ & $\begin{array}{l}K \\
\text { Mean (SD) }\end{array}$ \\
\hline $2 \mathrm{mg}(n=8)$ & $135.6(31.6)$ & $33.6(10.5)$ \\
$4 \mathrm{mg}(n=10)$ & $127.6(33.8)$ & $35.7(10.5)$ \\
Placebo $(n=18)$ & $133.8(31.5)$ & $30.8(9.9)$ \\
\hline
\end{tabular}

We observed significant changes within subject differences for the photopic $V_{\max }$ between the $4 \mathrm{mg}$ nicotine and placebo $(P=0.041)$ as well as the $2 \mathrm{mg}$ nicotine and $4 \mathrm{mg}$ nicotine $(P=0.023)$. Photopic b-wave implicit times showed no significant changes. Analyses of scotopic responses and OPs will also be presented.

\section{Conclusions}

Subjects displayed an increase in the photopic b-wave peak amplitude in the $2 \mathrm{mg}$ nicotine dosage and a decrease in the $4 \mathrm{mg}$ nicotine dosage $(P=0.041)$. Individual differences were dose related. The photopic b-wave is considered to be primarily a measure of ON-cone bipolar cells, and therefore, these results are consistent with findings from animal studies that have identified $\alpha 7$ nicotinic acetylcholine receptors on ON-cone bipolar cells. These data also corroborate previous studies of the effects of cholinergic agonists on ERG responses in cat and the effects of smoking on ERG responses in humans. To our knowledge, this is the first demonstration that nicotine affects retinal information processing in humans.

\section{Poster C17}

\section{The fast and slow oscillations of the EOG in diabetes}

Marilyn E. Schneck, Leslie Shupenko and Anthony J. Adams

School of Optometry, Vision Sciences Program, The University of California at Berkeley, Berkeley, CA, USA

\section{Purpose}

To assess the function of the RPE/photoreceptor complex in diabetes through measurement of the fast oscillation and slow oscillation of the EOG.

\section{Methods}

Thirteen visually normal subjects, 10 persons with diabetes without retinopathy, and 13 persons with mild-moderate retinopathy were tested. An LKC system (Gaithersburg, MD) was used for recording. Fairly standard EOG recording techniques were used. Silver/silver chloride electrodes were placed at the medial and lateral canthi of each eye with ground on the forehead. Saccade targets were 30 degrees apart. Ganzfeld luminance was $215 \mathrm{~cd} / \mathrm{m}^{2}$ and pupils were not dilated. First the fast (FO) and then slow oscillation (SO; Light Peak) of the EOG were recorded. The amplifier was set at $50 \mu \mathrm{V} / \mathrm{div}$, and the signal was bandpass filtered $(0.05-30 \mathrm{~Hz})$. Several parameters of each measure were considered: the Arden Ratio for the SO and P/T ratio for the FO, amplitude of the response (difference between voltages in the light and dark), and the peak and trough voltages.

\section{Results}

Sample size was small in this study, so we regard it as preliminary. Neither the Arden ratio nor the P/T ratio revealed any differences among groups (ANOVA $P=0.42, P=0.20$, respectively). However, the amplitudes, peak voltages of the slow oscillation, and fast oscillation showed significant differences between those with diabetes and those without. The normal/abnormal agreement between the SO and FO measures was only weak to moderate and varied among the measures. In general, SO and FO responses were not correlated.

Ratio measures were suggested to decrease variability of SO EOG results. The practice was adopted for the FO. These measures do reduce variability modestly. Nonetheless, ratio measures are insensitive to change in diabetes. When measures other than the ratio are examined, both EOG measures show diabetes-related reductions in amplitude and peak voltage. The modest agreement between the two measures is to be expected, given that the mechanisms that underlie the FO and SO differ. However, both RPE processes are affected in diabetes, even in the absence of retinopathy.

\section{Poster C18}

\section{ERG response smearing into 'unused' M-sequences} at fast stimulation rates

\section{R. P. Hagan, A. C. Fisher, M. C. Brown}

Department of Clinical Engineering, Royal Liverpool University Hospital, Liverpool, UK

\section{Purpose}

To determine the number of sequences (within an m-sequence family) that have a tangible signal (considering linear and nonlinear components) relating to visual stimulus run by a single m-sequence.

\section{Methods}

The ability of the m-sequence to encode and decode the electrical activity of several areas down just one recording channel has made the mfERG a reality. What makes this possible is that by shifting an $\mathrm{m}$-sequence (which is a pseudo binary random sequence) by one step a daughter m-sequence is created that has no correlation to the first mother sequence. At fast stimulus rates the time between sequence steps is not sufficient to contain all the response and part of the response may be captured in the neighbouring sequences (note, not neighbouring hexagons).

A single hexagon was used to stimulate the retina (to avoid mutual kernels) driven by a single 511 long $(n=9)$ sequence at 6 different rates. 20 normal volunteers participated and recording was made with thread electrode, with pupils dilated with $1 \%$ tropicamide. Stimulus had peak luminance at $120 \mathrm{~cd} / \mathrm{m}^{2}$ and was near $100 \%$ contrast with surround held at $60 \mathrm{~cd} / \mathrm{m}^{2}$. Ethical approval was obtained from the Local Regional Ethical Committee and all subjects gave informed, written consent.

\section{Results}

At the fastest rate ( $b p=16.67 \mathrm{~ms}$ ) there were 32 sequences easily identifiable as having power above the ambient noise. At this rate five clusters of signal were identified centred around the first order, second order, third order kernels as well as the second and third slice of the second order. Whilst other higher order kernels may be present they are not readily seen in this cohort of 20 subjects above background noise. At a slower rate (bp $=83 \mathrm{~ms}$ ) only one sequence was determined to have a significant response above the noise level. 


\section{Conclusion}

This practical data would suggest that it is impossible to run a 19 hexagon stimulus with a base period as short as $16.7 \mathrm{~ms}$ without clashes from higher orders and higher numbers of hexagons would have further clashes. It would also suggest that at a slower rate $b p=83 \mathrm{~ms}$ only one sequence need be excluded from the decimation for each stimulating hexagon. Overall this study suggests that short sequences are best used when there are a limited number of stimulating hexagons or when the stimulus is slowed down.

\section{Poster C19}

Transfer functions for rescaling electroretinograms obtained with two different translid DTL electrodes

\author{
Vaegan $^{1,2}$, A. C. Fisher ${ }^{3}$, Tom Wright ${ }^{4}$ \\ ${ }^{1}$ School of Optometry and Vision Science, University of \\ New South Wales, NSW 2052; ${ }^{2}$ Vision Test Australia, Eye \\ and Vision Research Institute, Sydney, NSW 2000; \\ ${ }^{3}$ Department of Clinical Engineering, Royal Liverpool \\ University Hospital, Liverpool UK; ${ }^{4}$ Department of \\ Ophthalmology and Vision Science, Hospital for Sick \\ Children, Toronto, Canada
}

\section{Purpose}

To compare the performance of two commercially available DTL ERG electrodes in a double blind trial using a method previously established by Vaegan et al. (ARVO 2008, Abstr \# 2225), which creates linear scaling transfer functions for both means and standard deviations of amplitudes and peak times. Methods

Two DTL electrode variants, the DTL plus Model D141 (Diagnosys LLC, Cambridge UK) and another fabricated by the Department of Clinical Engineering, Liverpool, UK, were used. Both are multi-filament ca $5 \mathrm{~cm}$ long, low impedance ( $c a$ $300 \mathrm{ohm}$ ) fibres with non-allergenic adhesive pads each end. The Liverpool model is in a closed sterile pack. The eye with each electrode was randomized. After $>7 \mathrm{~mm}$ dilation ISCEV standard bilateral ERGs and 19 area mfERGs were recorded in 6 normal persons and 5 patients with symmetrical bilateral disease. The data were sorted by electrode type named only as $\mathrm{A}$ and $\mathrm{B}$ and sent to ACF for interpretation and cursoring. To increase the correlation of the fitted functions, measures included components with the widest possible range. Amplitudes varied from the bright flash ERG to the 3 ring averages of the first order kernel mfERG. The two regression equations, constrained to pass through zero, of the relationship ( $\mathrm{A}=b \mathrm{~B}$ and $\mathrm{B}=c \mathrm{~A}$ ) of the means and s.d.s of each measure were calculated. The 'true' exponents were estimated by averaging the first and the inverse of the second exponent $((b+1 / c) / 2)$ while the error of estimate was given from $b-1 / c$. Coefficient of variation (amplitude exponent/s.d. exponent) or SNR of A and $\mathrm{B}$ were compared and the significance of the difference from a ratio of 1.0 calculated. Spreadsheets containing the amplitudes and times to peak of each component and their means and standard deviations (s.d.s) with each electrode (identified only as type A and type B) were sent to a third location for comparison. Responsibility for clinical procedures and masking of $\mathrm{A}$ and $\mathrm{B}$ was assumed by $\mathrm{V} ; \mathrm{ACF}$ independently performed the data analysis; TW unmasked identities A \& B and summarized the results. The identity of types A and B was then unmasked.

\section{Results}

Here we report the 3 amplitude measures: the mean mfERG P1, the scotopic bright flash a wave and the photopic bright flash $b$ wave. Measures from the two electrode types were both stable and reliable. Non-parametric analysis of each covariate by Kolmogorov-Smirnov technique ('measures of distribution') and Mann-Whitney for paired data did not reject the null hypothesis (for $\alpha=0.5$ ) that the samples of electrodes performed identically. Thus there is no indication that a transfer function other than 1.0 should be applied.

\section{Conclusion}

This study suggests that the DTL plus D141 and Liverpool variant electrodes perform similarly and that, with caution, could be used interchangeably. The complete experiment will use far higher numbers of test subjects and will establish whether a multivariate parametric statistical analysis will still support the conclusions.

\section{Poster C20}

Retinal functional and morphometric analysis of intravitreal triamcinolone acetonid injection in diffuse diabetic macular edema: optical coherence tomography and multifocal electroretinogram study

Fatih C. Gundogan, Serdar Memisoglu, Ali H. Durukan

Gulhane Military Medical Academy, Department of Ophthalmology

\section{Purpose}

To investigate the functional and morphologic changes after intravitreal triamcinolone acetonide (IVTA) injection in diffuse diabetic macular edema (DDME).

\section{Methods}

Twenty-four eyes with DDME were treated with an intravitreal injection of $4 \mathrm{mg}$ of triamcinolone acetonide. Visual acuity (VA), central macular thickness (CMT), mfERG, and intraocular pressures (IOP) were evaluated in the pre-injection period, 15th day, 3rd month, and 6th month after IVTA injection.

\section{Results}

Age and gender of the patients were not significantly correlated to LogMAR-VA ( $P=0.888$ for age, $P=0.192$ for gender), CMT $(P=0.282$ for age, $P=0.625$ for gender $)$, and $\mathrm{P} 1 / \mathrm{N} 1$ amplitudes/implicit times in mfERG at baseline $(P>0.05$ for age and gender). The correlation between LogMAR-VA and CMT at baseline was also not significant $(r=0.069$, $P=0.750)$. The VA increased significantly at the 15 th day $(P<0.001)$. The VA increase persisted until the $3 \mathrm{rd}$ month and decreased to pre-injection values at the 6th month $(P=0.324)$. CMT decrease was significant in the 15 th day $(P=0.012)$, but insignificant at the $3 \mathrm{rd}$ and 6 th months ( $P=1.000$ for both). The correlation between LogMAR-VA change and CMT change at the 15 th day was not significant ( $r=-0.043, P=0.843$ ). Moreover, the correlation of CMT change to $\mathrm{P} 1 / \mathrm{N} 1$ amplitudes/implicit times changes was not significant $(P>0.05$ for all $)$. 


\section{Conclusions}

The effect of IVTA injection on CMT and VA in DDME is reversible until the 6 th month. The insignificant correlation between the functional and morphologic tests is probably caused by irreversible macular dysfunction due to long duration of macular edema and primary neurophysiological effects of diabetes on the retina.

\section{Poster C21}

ERG Explorer: a software program for post-processing, analyzing, and report electrophysiological data

T. Strasser, R. Wilke, A. Messias, E. Zrenner

Institute for Ophthalmic Research, Centre for

Ophthalmology, University

\section{Purpose}

To develop and present a software application which (1) reads raw data from the electrophysiological systems from several manufacturers, (2) can evaluate and process the data according to ISCEV recommendations, (3) present the processed data in a standardized graphical report, and (4) make this data available for clinical and multicenter trial data management systems or statistical software packages.

\section{Methods}

The system is based on the Java Platform Enterprise Edition (Java EE) [1]. The application is divided into three major building blocks: a database for central storing of data, a server to provide an interface to different electrophysiological systems and automated report generation, and a client application for presentation and further processing of the recorded data. To allow the extension of functionality used for processing data, the well-known pattern pipes-and-filters [2] in combination with the Java Plug-in Framework (JPF) [3] is used. New algorithms can easily be added by dropping a new plug-in into the system. At present, algorithms like fast Fourier transformation and reconstruction, digital frequency filters, curve fitting, detrending, intensity-response-function, a-wave slope detection, signal-to-noise ratio estimation, and wavelet analysis are implemented. For the presentation of recorded data JasperReports [4] is used. It allows for a flexible design of reports based on customizable templates. Templates according to ISCEV Standards [5] and extended protocols are included. JasperReports also allows exporting data into many different formats including Excel or CSV. Additionally, the client application features the exchange of data with other applications by simple copy-and-paste from an Excel-like spreadsheet view. Currently we are providing an interface for the Espion E2 Electrophysiology system [6]; interfaces for LKC UTAS [7] and Roland Consult Reti-port [8] are being developed.

\section{Results}

The use of common open source frameworks allows for easy and quick adaptation of new algorithms for postprocessing electrophysiological data, the integration of new standards, outstanding reporting features and interfaces to existing devices or applications. Especially the tight integration in the study management system isTUmas [9], developed in Tuebingen, provides a unique way of exchanging electrophysiological raw data in multicenter studies via Internet.

\section{Conclusions}

The developed software is already successfully used in routine clinical examinations as well as clinical trials. Further features are also currently being implemented, which include the integration of additional electrophysiological systems and the implementation of new advanced algorithms for post-processing the data, e.g., wavelet analysis and noise estimation. An auxiliary accomplishment may be the establishment of a DICOM sub-standard to standardize the exchange of ocular electrophysiological data.

Acknowledgements A. Kurtenbach and H. Jägle, Department of Pathophysiology of Vision and Neuro-ophthalmology, Tübingen

\section{References}

1. Java EE, The Java Platform Enterprise Platform, http://java. sun.com/ja

2. Pipes and Filters, Pattern-Oriented Software Architecture, F. Busc

3. JPF, Java Plug-in Framework Project, http://jpf. sourceforge.net

4. JasperReports, http://jasperreports.sourceforge.net

5. ISCEV, International Society for Clinical Electrophysiology of Vision, http://www.iscev.org

6. Espion E2, Diagnonsys LLC, http://www.diagnosys llc.com

7. UTAS, LKC Technologies Inc., http://www.lkc.com

8. Reti-port, Roland Consult, http://www.roland-consult.de

9. Straßer T, Lotz G, Troeger E, Zrenner C, Peters T, Wilhelm B, Wilke R, Zrenner E (2008) An integrated system for workflow and data management in clinical trials. ARVO 2008 Annual Meeting, Fort Lauderdale, USA, 27.04.-01.05

\section{The Emiko Adachi Lecture 2008: Maculas, monkeys, metabolism, aging, and AMD}

\section{William W. Dawson}

University of Florida, Gainesville, FL, USA

Age-related macular degeneration (AMD) produces clinical signs that allow a split into "wet" and "dry" disease types. Among AMD patients, some authors claim that greater than $98 \%$ have the signs of the dry disease. Other surveys have shown that the incidence of AMD increases dramatically with age after 70 years and that after 80 years of age more than $15 \%$ of the older population show some evidence of AMD changes. The most common fundus signs (irregular local lesions, usually $<250 \mu \mathrm{m}$ ) are "drusen," identified as the "hallmark" of AMD by some authors. Drusen rarely extend out of the macular region, where they congregate progressively in different formations, from small punctate lesions to larger coalescing lesions with soft edges, often associated with pigmentary changes. Structures thought to be drusen have been described by light microscopy and by electron microscopy. Histological comparisons with the clinically identified drusen are difficult because the smallest visible clinical druse is approximately $12 \mu \mathrm{m}$. Advancing dry disease may show associated field 
losses, with retinal atrophic changes above local, visible atrophic changes in the choriocapillaris.

According to John and Shirley Sarks, after a unique series of longitudinal studies of Australian patients, wet disease including edema, retinal membranes, and hemorrhagic events often follow hard-soft drusen development and expansion. The formation of wet disease in the absence of drusen is controversial. Other longitudinal data is rare and poorly quantitative.

The foundation of abnormal physiology leading to human drusen, membranes, and bleeding is difficult to establish, in the absence of hard longitudinal data. The predictable development of pathology associated with (PE) pigment epithelium/choroidal abnormalities would encourage the understanding in the first events leading to the well-known clinical signs. A surrogate would be of great value.

Some closed-colony rhesus monkeys (Macaca mulatta) may provide the foundational data needed to uncover events in the early development of AMD. We discovered signs of AMD in the rhesus monkeys from the Caribbean island of Cayo Santiago in 1984. The island had been populated in 1938 and the gene pool became concentrated during the next fourteen generations of inbreeding. A sample of 50 animals was moved to Florida in 1994, and with selective breeding, now $70 \%$ show signs of AMD by 10 years of age. The fundus signs include pigmentary change, hard drusen, soft and confluent drusen, and a very rare case of wet, hemorrhagic disease. The histology of the rhesus drusen-eye shows PE-Bruch's membrane lesions similar to those described as drusen for humans and appearing primarily to be filled with cell debris. Ultrastructure of the human and rhesus drusen are nearly identical. The human visual function altered earliest by AMD has been demonstrated to be peripheral $\left(12^{\circ}-20^{\circ}\right)$ delayed dark adaptation kinetics. The same change in receptor function has been demonstrated in the rhesus surrogate. The Florida-Cayo surrogate has encouraged the further identification of drusen development and associated cellular changes.

The structure of drusen, their clinical appearance, and the fact of receptor outer segment shedding encourages hypotheses involving abnormal lipid movement and/or lipid geography. Examinations of retinal histopathology over the last 30 years and the routine methods of tissue preparation, in the vision community, point to the almost uniform application of organic solvents which would remove lipids from the processed tissue for dehydration and embedment. There have been fewer than six retina studies in any year since 2000 where modern freeze sectioning techniques have been applied and lipids have therefore been retained in the tissue. In contrast, in 2006 over 18,000 publications from light and electron microscopy utilized organic solvents in retina tissue preparation. Using monkey tissue, Anderson, Curcio, and Dawson with modern freeze technology found numerous lipid filled PE cells in the old adult-drusenoid rhesus eye using ORO lipid specific stain. The paper reported singles and clumps of lipid filled PE cells in tissue shown to have "clinical appearing drusen." However, the number of drusen seen in the histological sections was relatively small (1/300) compared to lipid-PE cells. Subsequently, we have developed a method using tandem scanning mass spectrometry of flat retina samples to plot the geographic distribution of lipids in donor human and Florida-Cayo monkey retinas. The fundus flatmount geography always shows various lipids, but mainly uniformly distributed cholesterol and phosphatidylcholine (PC) variably around the macula with occasional evidence in the periphery. The concentration and density of PC appear to be roughly correlated with clinically visible drusen and age-related progression.

The availability of the Florida-Cayo surrogate has provided new data and opened up new channels for investigation and leads to an interesting hypothesis. The Florida-Cayo monkey with drusen has a functional loss after bleaching, much as in human patients. The spatial aging distributions of lipids and drusen are similar in AMD affected eyes, human or monkey. The most frequently found macular lipid (PC) is critical in the formation and degradation of receptor outer segments. We have been successful in increasing the frequency of AMD affected individuals by selective breeding.

The overall hypothesis which we have been encouraged to make is that AMD begins as a macular waste disposal problem initiated by metabolic stress following decline of the conduit functions of the PE cell membranes. The main waste is phosphatidylcholine.

In the future, we hope to produce larger numbers of AMD animals so that the research efforts may be expanded. We will do this by super-ovulation, artificial insemination, and surrogate mothering where male and female contributors have a strong history of AMD development. We will begin new experiments by manipulating circulating lipids in an attempt to modify PE-choroidal lipid transport. Further, we will attempt to manipulate choroidal circulation in the presence of stable circulating phosphatidylcholine. New human clinical equipment for the very early evaluation of fundus lipid chemistrygeography may become possible through the development of anti-stokes infrared Raman-shift spectrometry. 